\title{
EXPLORING SPACE
}

EFFECTS OF ENVIRONMENTAL STIMULI ON SELF-DISCLOSURE

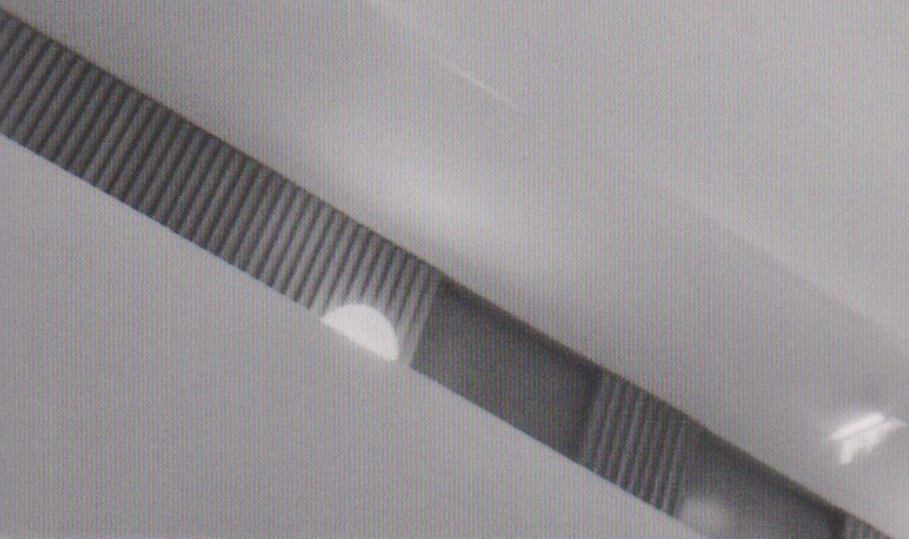




\title{
EXPLORING SPACE
}

\section{EFFECTS OF ENVIRONMENTAL STIMULI ON SELF-DISCLOSURE}

\author{
VANESSA OKKEN
}


Thesis, University of Twente, 2013

(C) Vanessa Okken

ISBN: 978-90-365-0738-7

Cover picture by Arjo Stokman

Cover design by Vincent Lutje Wooldrik

Printed by Ipskamp Drukkers B.V., Enschede, the Netherlands 


\section{EXPLORING SPACE}

\section{EFFECTS OF ENVIRONMENTAL STIMULI ON SELF-DISCLOSURE}

PROEFSCHRIFT

ter verkrijging van

de graad van doctor aan de Universiteit Twente, op gezag van de rector magnificus, prof. dr. H. Brinksma,

volgens besluit van het College voor Promoties in het openbaar te verdedigen op donderdag 14 november 2013 om 12:45 uur

door

Vanessa Simone Okken

geboren op 2 januari 1981

te Hoogeveen 
Dit proefschrift is goedgekeurd door de promotor prof. dr. A. Th. H. Pruyn en assistent-promotor dr. T. J. L. van Rompay. 


\section{Samenstelling promotiecommissie}

Promotor: $\quad$ Prof. dr. A. Th. H. Pruyn

Assistent-promotor: $\quad$ Dr. T. J. L. van Rompay

Leden: $\quad$ Prof. dr. A. E. van den Berg

Prof. dr. ir. A. O. Eger

Prof. dr. V. Evers

Prof. dr. E. Giebels

Prof. dr. P. P. M. Hekkert

Prof. dr. C. P. M. Wilderom 



\section{Contents}

Chapter 1

Chapter 2

Chapter 3

Chapter 4

Chapter 5

Chapter 6

Chapter 7

References

\author{
Chapter 5
}

interaction

When the world is closing in: Effects of perceived room brightness and communicated threat during patient-physician interaction

A spacious view: Influences of the physical and online environment on self-disclosure

General Discussion

Samenvatting (Summary in Dutch) 



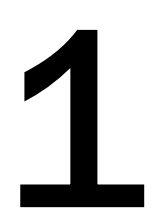

General Introduction 
"The most important things lie too close to wherever your secret heart is buried, like landmarks to a treasure your enemies would love to steal away."

(Stephen King, 1982)

\section{Introduction}

During our daily life we are surrounded by many service providers. For instance, when you are travelling, you can contact an airline company or a car rental company or may stay in a hotel; when you have fallen ill, you go to a general practitioner or a hospital and when your mental health is compromised, you contact a counselor or a psychotherapist. All these and many other service providers have one thing in common; as a result of having to formulate their service request, the client has a very active role in the process of service provision. However, although a client proceeds to articulate his or her needs, which lies at the basis of good service provision, it may still be difficult to correctly and completely formulate their request. Arguably, making your request clear to the service provider is easier when visiting a hotel than when consulting a mortgage broker or an insurance agent. This difficulty of communicating a service request can become even more problematic when it concerns personal information or a specific request for medical assistance. But before elaborating more on these issues, let me present a few examples that will help in understanding the difficulties.

First, imagine yourself being in the following situation:

"You have moved to a new city and today you are visiting your new general practitioner for the first time. When entering the consultation room, you immediately see and feel a difference with the office of your previous GP. You introduce yourself and are invited to sit down at the desk. The doctor asks you about the reason for your visit. You find it hard to discuss your complaints. You want the consult to be over as quickly as possible and try to keep things short. After the doctor discussed his treatment plan and hands you a prescription, you leave the consultation room. Once outside the room, you take a deep breath and feel liberated."

What could have caused your difficulties in expressing yourself and talking to this physician? Was it because the health problems were embarrassing? Did the doctor's tone of conversation come across as threatening? What part did the consultation room itself play in this particular situation? Now, let's take a look at a somewhat different situation: 
"You recently had an intake with a lifestyle coach. During this intake the coach explained the coaching procedure and you filled in an intake questionnaire regarding your personal life. You are now having the second meeting with the lifestyle coach during which you will discuss your questionnaire results. The coach explained that during this meeting you are expected to elaborate more on your answers, providing her with the necessary information to choose a fitting approach. The coach invites you to enter her office and sit down, after which she starts discussing your questionnaire answers. Even though it was your own plan to consult a lifestyle coach, you are taken a bit aback by the feedback the coach gives you. While the first meeting was pleasant and open, this second meeting does not feel pleasant at all. When she asks you a question, you feel like your back is against a wall and you are forced to give information you don't want to share. To be honest, you feel that there is no purpose explaining yourself to this person but you feel that it would be rude to stop the meeting prematurely. After this second meeting, you leave the office with a very negative feeling and you strongly consider not to continue meeting with this coach."

What went wrong during this second meeting with the lifestyle coach? Were you insulted by the feedback? Was it the straightforward approach of the coach? Would you have preferred some more breathing space during this confronting conversation? Finally, an example in the virtual world; imagine yourself approaching a dating site in your search for a new partner.

"You have been single for over a year and you are feeling lonely. You have met some people in bars and have had several blind dates that were arranged by your friends, but it never was a good match. In your search for a new partner who has similar interests and hobbies, you go online and find a dating site where singles such as yourself can meet and communicate with each other. However, before you can enter the website and contact others, you have to register as a member. You start filling in the registration form but while answering the questions you feel limited in the possibility to give a fitting answer to the questions. As a result, you become less enthusiastic about your membership. You read some of the next questions and don't feel like answering the questions anymore. Without completing the registration form, you close the website and continue your search elsewhere."

What these three examples have in common is that in all these instances the client is the person who voluntarily contacted and met with the service provider. The client also knew beforehand that during the meeting personal matter needed to be discussed, a process also referred to as self-disclosure (Cozby, 1973). Despite this knowledge, it was still difficult to share personal information in that particular situation. What the examples also have in common is that the conversation takes place in a (physical or online) service environment. Many factors that are present 
during such a conversation can impact the level of self-disclosure of the client, including factors related to both individuals and the conversation itself. In this dissertation, the focus is on the environment in which the conversation takes place and how this environment impacts how people feel and behave. To be more specific, the studies discussed examine whether environmental factors can impact self-disclosing behavior and to what extent this relationship is influenced by spaciousness perceptions of the environment.

\section{Overview of the present dissertation}

Since the effect of environmental factors on self-disclosing behavior via perceived spaciousness is unexplored territory, the studies presented in this dissertation are a first step towards filling that void. Knowledge about how perceived spaciousness can be influenced by environmental factors can help in constructing and decorating environments, so that visitors feel free and uninhibited rather than confined and constricted. In turn this can positively influence both affective experience and behavioral responses, overall creating a more positive service experience.

In Chapter 2, an overview will be given of research on self-disclosure, discussing several factors that influence this communicative behavior. Furthermore, research on environmental factors is discussed and a classification is presented of different groups of environmental factors.

In Chapter 3, two experiments are presented that examine the effects of architectural and interior design elements in a healthcare environment. More specifically, the effects of room size and desk size are investigated during patientphysician interaction in a consultation room. Additionally, in the first study personality differences are taken into consideration, examining people's individual level of reservedness (i.e., shyness). In the second study, the conversational tone is taken into account, comparing the effects of positive and negative conversations.

Chapter 4 demonstrates that the effects found in the experiments presented in the third chapter can be replicated in a tangible environment in which, besides disclosing intentions, actual disclosing behavior can also be measured. In the study presented in this chapter both room size (architectural space) and desk size (interior design) are manipulated to impact spaciousness impressions during interviews on intimate life style related topics. The interviews were recorded on videotape so that both verbal and nonverbal behavior could be examined. 
Chapter 5 addresses the question whether atmospherics can be used to impact spaciousness perceptions and in turn impact self-disclosure. The study presented in this chapter describes an experiment in which room brightness was manipulated in a fictive patient-physician encounter varying in communicated threat.

Chapter 6 deals with the question of how online conversations are impacted by both physical and online environmental manipulation. In the studies reported in this chapter, room brightness and website design were manipulated to influence feelings of spaciousness during computer tasks.

The dissertation concludes with a general discussion in Chapter 7. An overview of the main findings will be presented, and the implications, limitations and directions for future research are discussed.

The methodological chapters presented in this dissertation are all based on articles that currently are published in or submitted to scientific journals. To ensure that all chapters can be read independently from each other, the introductions to each chapter show some overlap. 
In Chapter 1 the key concepts of this dissertation, environmental factors and selfdisclosure, were briefly introduced using three examples. In Chapter 2 an overview will be presented of previous research on these topics. The chapter will start with an overview of the factors that have been shown to influence self-disclosure, after which a closer look will be taken at the influence of environmental factors. Furthermore, a classification will be presented, which subdivides environmental factors into four categories: architecture, interior design, atmospherics and, with respect to online communication, website design. 


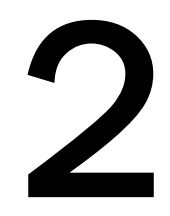

Theoretical background 
"As long as man must live in a world of walls, furniture, doors and fences, there is good reason to study how they influence his behavior."

(Robert Sommer, Studies in personal space, 1959)

\section{Introduction}

The sharing of personal information with another person is also called selfdisclosure (Cozby, 1973). Although self-disclosure often brings to mind a conversation between two people, it is not necessarily limited to dyadic conversations and can also occur within a group of people. Furthermore, although both the term and description may suggest that self-disclosure only occurs in faceto-face conversations and is limited to oral communication, writing down personal information that will be read by another person (i.e., written disclosure) is also considered self-disclosure (Barak \& Gluck-Ofri, 2007; Gifford, 1988; Ignatius \& Kokkonen, 2007; Joinson, 2001; Joinson, Paine, Buchanan \& Reips, 2008; Jourard \& Friedman, 1970). The personal information discussed may concern facts about oneself, personal opinions and attitudes, or information about moods and emotions (Omarzu, 2000). People benefit from self-disclosure because they are rewarded with social approval, shared intimacy, stress-relief, gained social control and / or identity clarification (Derlega, \& Grzelak, 1979). In addition, self-disclosure has also been linked to health benefits, with more disclosure of traumatic experiences improving health outcomes (Pennebaker \& Beall, 1986; Pennebaker, Kiecolt-Glaser \& Glaser, 1988). On the other hand, disclosing personal information is also subject to several risks, such as rejection by the listener, reduction of one's autonomy and personal integrity or loss of control or self-efficacy (Omarzu, 2000). Self-disclosure intentions are higher when the risks are lower and when the discloser is certain that the shared information will not be used against him / her. As a result, selfdisclosure is considered to be most appropriate towards spouses, close friends and relatives and least appropriate towards strangers (Chaikin \& Derlega, 1974; Jourard \& Lasakow, 1958; Mathews, Derlega and Morrow, 2006; Morton, 1978).

However, there is another situation in which self-disclosure is necessary. It is important in many service settings where good-quality client input is essential to acceptable service delivery. The service provider to a large extent depends on the disclosed information of the client. Thus, the better the client dares to disclose their service requests, the better the service provider can deliver the requested service. Conversely, when the client is hesitant and discloses less information, the service provider needs to fill in the blanks and is unsure whether the provided service is tailored correctly to the client's needs. In turn, whether the client's needs are met impacts the service quality evaluation, client satisfaction and the client's loyalty to 
the service provider. In other words, stimulating client self-disclosure can improve the process of service delivery and encourage recurring visits.

In healthcare settings, such as the examples presented in the first chapter, selfdisclosure is a key factor in the success of a treatment procedure and a lack thereof on the patient's behalf may prevent the physician from forming an accurate picture of which symptoms are experienced and of the patient's complaints and medical history. On the contrary, a patient that feels free to disclose more information in general, and more personal information in particular (e.g., information that might seem irrelevant from the patient's point of view but that may turn out to be relevant to the diagnosis), enables a physician to make a more accurate diagnosis and prescribe a better patient-tailored treatment (Cegala, Gade, Lenzmeier Broz \& McClure, 2004). In turn, such a positive experience with a healthcare provider will encourage the patient to more readily seek help in the future and to also return to this specific physician because of the previous success.

Next to face-to-face communication, the internet provides us with another still growing environment in which we can communicate with others: the online environment. Besides presenting an easy way to interact with friends and family, the online environment is also a territory well explored by service providers. With this still somewhat new trend, the necessity for people to self-disclose online steadily increases. For instance, communicating with physicians or counselors during online consults, interacting with fellow members on an online forum and providing extensive information in online profiles are nowadays common activities. The growing popularity of the online environment has also attracted the attention of researchers and, besides the effects of the content of websites, the effects of website design have also been examined. Researchers investigating website design often build on models that stem from physical environmental research (e.g., Kaplan \& Kaplan, 1988; Mehrabian \& Russell, 1974) to explain found effects (Eroglu, Machleit, \& Davis, 2001; Koo \& Ju, 2010; Richard 2005; Rosen \& Purinton, 2004), suggesting that both environments have a similar impact on our affective experience and behavior. This calls to question whether online self-disclosure can also be influenced by website design elements and whether experienced spaciousness also plays a role in this more or less two-dimensional way of communicating via a computer screen.

When someone is unwilling to self-disclose during a conversation with a close one, the result can be the end of the conversation but, more likely, the topic of conversation will change. During conversations in service settings however, a change of topic is far more unlikely. Instead, the professional service provider will probably keep questioning the client in order to fully understand the service question and to complete the conversation. As a result, the client may feel 
constricted in the conversation and the conversation will reach a low point. One can imagine that such a conversation is a negative experience for the client, which lowers their willingness to return to this service provider. It thus seems relevant to further explore how to promote or facilitate self-disclosure in both physical and online service environments. After reviewing the literature on self-disclosure, it will be argued that in addition to factors related to the client, the service provider and the conversation itself, the environment in which the conversation takes place may foster self-disclosure by creating the right 'atmosphere'. The studies reported in this dissertation set out to address this hypothesis, thereby advancing understanding of environmental impacts on communicative behavior. First, a brief overview of previous research addressing self-disclosure and the factors involved.

\section{Factors influencing self-disclosure}

Discussing personal information can be considered difficult due to the risks that are involved in sharing sensitive or perhaps embarrassing information about yourself (Omarzu, 2000). Furthermore, research shows that the emotional state of the discloser influences their disclosure tendencies (Forgas, 2011; Ignatius \& Kokkonen, 2007). A discloser with a relaxed, worry-free state of mind more easily discloses information then a discloser with a worried, anxious state of mind.

In addition to such situational factors that impact disclosure tendencies, the level of self-disclosure can also vary between people due to personality traits. Some people experience more difficulties expressing themselves in social contexts than others. Research shows that reservedness, shyness and need for approval influence selfdisclosure tendencies (Riley, Cozby, White \& Kjos, 1983). More specifically, people with high reservedness have more difficulty engaging in social interaction in general, and disclosing personal information in particular, compared to people with low reservedness.

Characteristics of the conversational partner can also evoke or reduce selfdisclosure. For instance, research shows that factors such as gender and age influence the amount of self-disclosure during conversations (Cappella, 1981; Strassberg, Anchor, Gabel, \& Cohen, 1978). Self-disclosure appears to be more frequent when the conversational partner is of the female sex and / or of the same age. Besides such demographic factors, the relationship with the other person is an important factor. As mentioned earlier, self-disclosure is considered to be most appropriate towards spouses, close friends and relatives and least appropriate towards strangers (Chaikin \& Derlega, 1974; Jourard \& Lasakow, 1958; Mathews, Derlega and Morrow, 2006; Morton, 1978). In line with this finding, results of 
Derlega, Winstead, Mathers and Braitman (2008) show that disclosure is evoked by factors such as trust and involvement. In addition to familiarity, liking also appears to have a positive relationship with self-disclosure. Results of a meta-analysis of Collins and Miller (1994) show that people tend to disclose more information to those whom they initially like. Overall, these results show that having a good relationship with another person increases the willingness to self-disclose.

Another important factor influencing self-disclosure is the conversation itself, since self-disclosure concerns sharing information that is personal. As mentioned earlier, this involves risks for the discloser, such as rejections by the listener, loss of control or reduction of personal integrity (Omarzu, 2000). In addition, topics that aren't regularly discussed, such as feelings and emotions, can be a lot harder to put into words.

Next to the topic of conversation, the distribution of disclosure between both conversational partners (i.e., the level of reciprocity) can also impact self-disclosure tendencies. Research shows that a high level of reciprocity (i.e., a more equal distribution of disclosure) is conducive to self-disclosure, especially when communicating with strangers (Morton, 1978). When communicating with close ones, the level of reciprocity is often high. Both people share equally in order to strengthen their bond. However, during conversations in service environments, the information flow is more one-sided; the client is expected to share much sensitive information while the service provider doesn't disclose. The client is thus the only one exposed to the risks of disclosure, which can reduce the willingness to selfdisclose. Research in the field of counseling shows that increased disclosure of the service provider, or counselor, positively affects the amount of disclosure of the client (Taylor \& Gill, 1983). In addition, a study by Nilsson, Strassberg and Bannon (1979) shows that counselors disclosing more personal information during sessions are judged more favorably than counselors who do not disclose personal information. Interestingly, the results also showed that the level of disclosure of the counselors did not affect judgments of their professionalism. This shows that 'balancing the scales' with respect to self-disclosure will not harm the integrity of the counseling session.

Based on the above, one can expect that improving client self-disclosure also improves the overall flow of the conversation. However, the discussed factors are difficult to improve; the risks of disclosure will always remain to some extent, service providers will almost never be considered a close one and information flow in service settings is by necessity unbalanced. Therefore, the focus of this dissertation is on yet another factor that is always present; the environment in which the conversation takes place. The environment is also known to impact both the affective experience and behavior (Dijkstra, Pieterse \& Pruyn, 2006; Pressly \& 
Heesacker, 2001; Ulrich, Zimring, Zhu, DuBose, Seo, Choi, Quan \& Joseph, 2008). In addition, it can be more easily manipulated and adjusted in accordance with the purpose of a room. Therefore, this chapter will continue with an overview of research on environmental factors.

\section{Environmental influences}

A growing body of research provides evidence for the influence of a number of environmental factors such as architectural factors, room layout, the presence of decorative objects, lighting conditions, music and the use of color on both the affective experience and behavioral responses (Pressly \& Heesacker, 2001; Turley \& Milliman, 2000; Valdez \& Mehrabian, 1994). For instance, Valdez \& Mehrabian (1994) examined the effects of different colors on emotions and showed that green colors were most arousing, while purple-blue and yellow-red were least arousing. In addition, research findings also testify to the importance of the physical environment for self-disclosure (Chaikin, Derlega \& Miller, 1976; Cohen \& Schwartz, 1997; Jourard \& Friedman, 1970; Lecomte, Bernstein \& Dumont, 1981; Sundstrom, 1975). For example, Jourard and Friedman (1970) examined the effects of interpersonal distance during communication. Their results show that when the physical distance within the dyad decreases, so does the extent of selfdisclosure.

There are several explanations of why environmental factors influence our feelings and behavior. One such explanation is that creating a comfortable or pleasant environment facilitates self-disclosure because such environments make people feel comfortable and at ease, which in turn induce disclosure tendencies (Chaikin, Derlega \& Miller, 1976; Miwa \& Hanyu, 2006). For instance, Chaikin, Derlega and Miller (1976) investigated the effect of room environments on self-disclosure in counseling settings. They compared a "cold", non-intimate room (consisting of bare walls and fluorescent lighting and no decorative items) with a "warm", intimate room (pictures on the walls, cushioned furniture, floor rug, soft lighting). Their results showed that self-disclosure was significantly higher in the intimate room than in the non-intimate room. Another, somewhat related, explanation is that creating a more home-like environment will make people feel at ease, which in turn will increase self-disclosure levels (Gifford, 1988). Gifford (1988) investigated the effects of lighting and room decorations on interpersonal communication and showed that brighter lighting and a more home-like decor made respondents feel more comfortable, in turn stimulating communication. 
Nevertheless, determining how to create a pleasant environment can be considered challenging when one takes into account that pleasure is determined by a diversity of factors inside the room that range from large-scale furniture pieces to small decorative and personal objects (Verhoeven, van Rompay \& Pruyn, 2007). Furthermore, pleasure is subject to individual differences and, to a large extent, depends on one's personal taste and distaste of, for instance, different decorative styles and different color schemes. As a result, it is difficult to pinpoint which factors within an environment should be attended to and how they should be altered.

Another factor that impacts how a room as a whole comes across is the experienced spaciousness within that room, a more straightforward concept that has also been shown to impact self-disclosure (cf. Okken, van Rompay \& Pruyn, 2012; Okken, van Rompay \& Pruyn, 2013; Sundstrom, 1975). However, although research has demonstrated effects of spaciousness-related factors such as room size on self-disclosing behavior, experienced spaciousness has not yet been examined as a possible mediator in such relationships. Therefore, in this dissertation it is argued that environmental factors influence self-disclosure via experienced spaciousness. The fact that a possible mediating effect of experienced spaciousness is not yet investigated can be considered somewhat remarkable. Consider for instance the first time you enter an unknown environment such as a consultation room of a specialist in a hospital. Whether the environment comes across as spacious or not is usually one of the first things that are noticed upon entering such an unfamiliar room. Even without having noticed all the different isolated factors present in a room, the room as a whole can trigger a certain amount of spaciousness. Before elaborating more on the concept of spaciousness, first a taxonomy of environmental factors is presented.

\section{Classification of environmental factors}

Several researchers have classified the physical environmental factors into different categories (Baker, 1986; Bitner, 1992; Harris, Ross, McBride \& Curtis, 2002; Turley \& Milliman, 2000). For instance, Baker (1986) distinguishes between three categories; social cues, design cues and ambient cues. Bitner (1992) on the other hand distinguishes between the space / function of the environment, the ambient conditions and the used signs, symbols and artifacts. Furthermore, Harris et al. (2002) subdivide environmental factors into architectural features, interior design features, ambient features, maintenance / housekeeping and social features. These are just a few classifications used in environmental research. The chosen classification to a large extent depends upon a researcher's interests. For example, maintenance and housekeeping are of such importance in hospital environments 
that they are distinguished as a separate category by Harris et al. (2002), whereas classifications in consumer research in service and retail environments (Baker, 1986; Bitner, 1992) has no such category. Furthermore, the existing classifications are not unanimous as to how the environmental factors should be classified. Whereas Harris et al. (2002) sees color as an interior design feature, Bitner (1992) places color under the ambient conditions. Due to this lack of coherence in the existing classification, throughout this dissertation an alternative classification will be used (see Figure 2.1), distinguishing architectural factors, interior design factors and atmospheric factors. An additional alteration to existing models of environmental factors influencing behavior is the addition of the online environmental factors to the model, since social interaction not only occurs face-toface but also via the internet.

The chosen environmental factors in the taxonomy stem from existing models along with environmental factors that will be addressed in this dissertation. Besides the factors presented in the taxonomy, there are many more factors to think of per category. Architectural factors concern all factors influencing the actual size and measurements of a room, such as room size, ceiling height, room measurements. Interior design factors concern the design of the room in terms of the items present in the room and the layout; the use and position of different furniture pieces, but also smaller decorative items that can be placed throughout the room. Atmospheric factors are to a large extent intangible factors that influence the atmosphere of the entire room such as used color schemes, lighting conditions, room brightness, odor and room temperature. This classification will be used to discuss literature on environmental factors related to spaciousness.

\section{Spaciousness}

Evidence for the importance of spaciousness in environments follows from a study by Haytko and Baker (2004) addressing experienced spaciousness of shopping malls. They interviewed people visiting the mall and many respondents brought up spaciousness as an important determinant of shopping pleasure. In addition, Hur, Nasar, and Chun (2010) investigated satisfaction of inhabitants of different neighborhoods. Their results showed that perceived spaciousness (or openness) is a key factor in determining neighborhood satisfaction. In addition to such public environments, room environments can also come across as more or less spacious due to several environmental factors including horizontal floor area, boundary permeability, wall roughness and light (Stamps, 2010; Stamps \& Krishnan, 2006). 


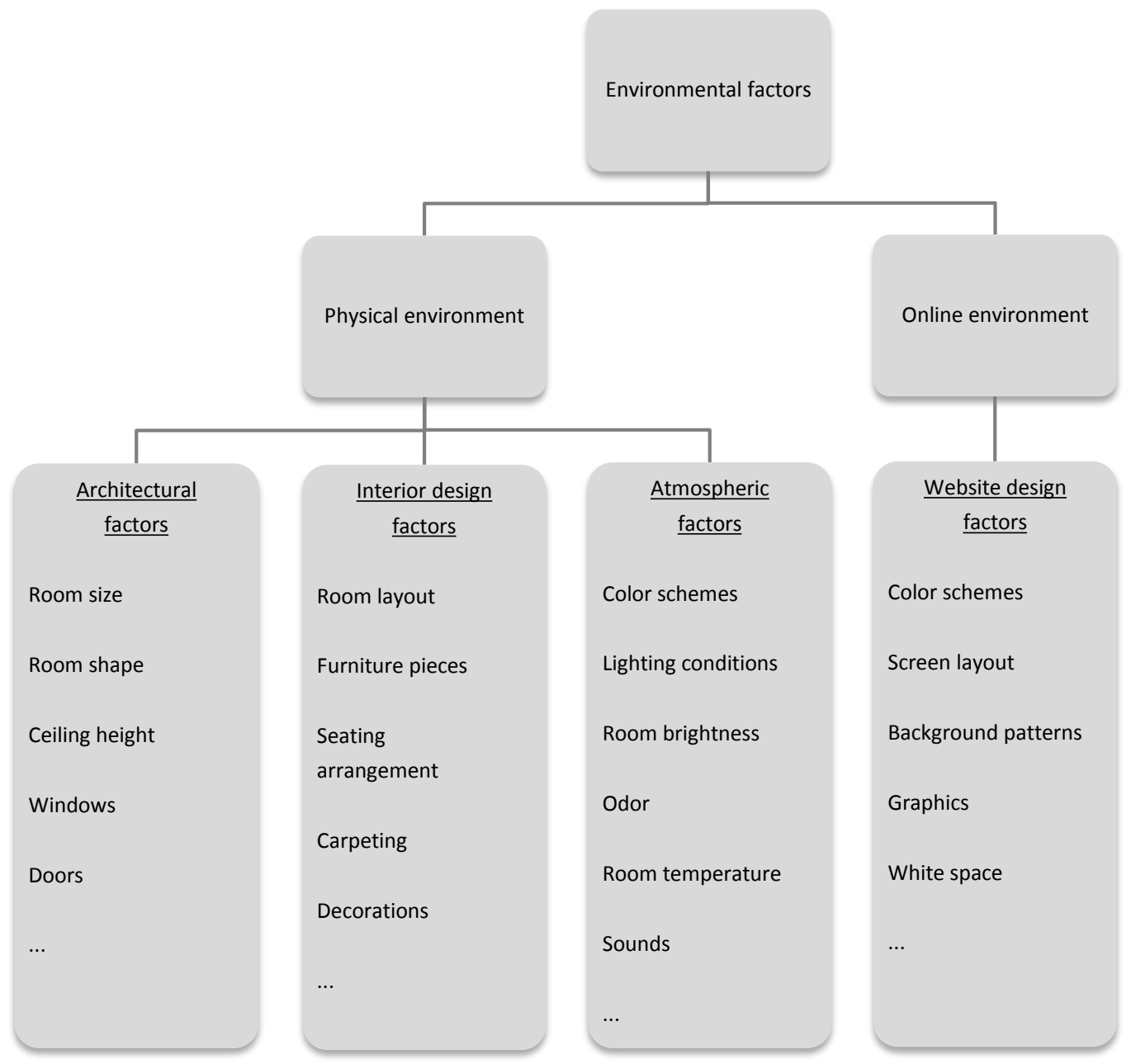

Figure 2.1. Taxonomy of environmental factors.

Research in the area of consumer behavior suggests that spaciousness is important because it inspires feelings of freedom (Meyers-Levy \& Zhu, 2007; Levav \& Zhu, 2009). Meyers-Levy and Zhu (2007) investigated the effect of ceiling height on problem solving and showed that a high, as opposed to a low, ceiling activated more feelings of freedom and spaciousness and induced more creative strategies for problem solving tasks. In addition, Levav and Zhu (2009) studied the effects of spaciousness in supermarkets and showed that narrower aisles activate feelings of confinement, which consumers often counteract by making more varied product choices (as a means to regain freedom). The findings of these studies suggest, in line with embodiment research, (IJzerman \& Semin, 2010; Williams \& Bargh, 2008) that experiencing restrictions of physical space invokes feelings of restrained 
psychological space. As a result, such negative feelings of restraint can cause reactance, which emerges as a refusal to cooperate or comply with behavioral norms or to perform expected behavior. In shopping environments reactance induced a more varied product choice, whereas during a conversation (during which self-disclosure is expected) it can result in a refusal to self-disclose. The positive effects of experiencing spaciousness, and perhaps of equal importance the negative effects of experiencing confinement, described above, call to question whether disclosure tendencies are also influenced by spaciousness perceptions and if so, which environmental factors impact experienced spaciousness.

\section{Physical environmental factors influencing spaciousness}

\section{Architectural factors}

When discussing factors influencing experienced spaciousness, perhaps the most logic place to start is with factors that impact the actual size of the room. Thiel, Harrison and Alden (1986) indeed argue that spatial enclosure is determined by the architectural features of a room; the ceiling and the walls that create the boundaries of the room. As discussed earlier, findings of Meyers-Levy and Zhu (2007) show that ceiling height impacts feelings of freedom, with a higher ceiling generating more perceived freedom. Furthermore, research confirms that room size impacts experienced spaciousness (Baird, Cassidy \& Kurr, 1978; Sundstrom, 1975). Sundstrom (1975) examined the influence of crowding on stress and found that smaller room size (i.e., limited physical space) induced crowding perceptions. Results of Baird, Cassidy, and Kurr (1978) also show that respondents preferred higher ceilings. In a similar vein, Stamps (2011) showed that rooms with larger horizontal area (i.e., larger room size) are perceived as more spacious. In addition to the size of a room, the shape of it can also impact spaciousness perceptions, sometimes even without changing the actual measurements of a room (Sadalla \& Oxley, 1984; Stamps, 2011). For example, Sadalla and Oxley (1984) investigated room size perceptions of different rectangular and square rooms and show that more rectangular rooms were judged as larger than less rectangular rooms of equal size. These findings were confirmed by Stamps (2011) who also found that spaciousness scores were higher for more rectangular spaces. These results show that not only the actual size of the room, but also the shape of the room impacts spaciousness perceptions and that rooms of equal size can differ in terms of experienced spaciousness. 


\section{Interior design factors}

Next to architectural features of built environments, interior design factors may also influence perceived spaciousness. Findings of particular relevance to the current context of self-disclosure address the relationship between the seating arrangement and self-disclosure (Albert \& Dabbs, 1970; Jourard \& Friedman, 1970; Sommer, 2007; Yildirim \& Akalin-Baskaya, 2007). Yildirim \& Akalin-Baskaya (2007) studied the effects of seating arrangements in a restaurant on customer satisfaction and found that larger distances between the tables yielded more positive responses. Similar results were found by Albert and Dabbs (1970) studying the influence of interpersonal distance on the effect of persuasive communication. Their results show that when the interpersonal distance was decreased, persuasive messages of the experimenter were less likely to be accepted by the participants. These results show that a decrease in the physical space between two people increases negative feelings of confinement, in turn lowering disclosure tendencies. As mentioned earlier, such negative feelings cause reactance which may impact disclosure tendencies. Results of a study by Jourard and Friedman (1970) confirm that a more intimate seating arrangement, decreasing physical distance between the experimenter and the participant, causes a decrease in the amount of selfdisclosure.

In addition to social density, that can be caused by factors such as room size, room layout and furniture arrangements, one can also experience insufficient spaciousness due to the amount of lifeless objects in the room (e.g., spatial density). Findings in the field of consumer research show that experiencing limited space in store environments due to spatial density significantly decreases shopping satisfaction (Machleit, Eroglu \& Mantel, 2000). In addition, results of Stamps (2011) show that the amount of free horizontal area in a room is key factor in determining experienced spaciousness. In other words, a large room with many furniture pieces and decorative items has limited free floor area and can therefore come across as less spacious than a smaller room with much less furniture inside.

However, taking this knowledge to the extreme and designing a room with little to no objects inside can come across as abnormal, in turn making visitors feel confused or uncomfortable. In addition, such an empty environment leaves people with little to no tools for estimating the size of the room which may bias the experienced spaciousness. Of interest in this line of reasoning are findings of Stamps and Krishnan (2006) showing that a completely empty room actually comes across as less spacious than when the walls are lined with bookcases. These results suggest that people use the furniture pieces inside the room as aids to establish room size and the amount of space. 


\section{Atmospheric factors}

In addition to the tangible factors discussed up to this point, intangible room atmospherics such as color and lighting have also been shown to impact spaciousness perceptions (Stamps, 2011) and related feelings and behaviors. Acking and Küller (1972) repainted patient dayrooms of a hospital in different colors and their results demonstrate that a white room was judged as most open, compared to light-green and dark-green rooms. Kwallek (1996) investigated the effect of color in office environments and found similar results; a white wall color received highest spacious scores, in comparison to green and red wall colors. Research by Oberfeld, Hecht and Gamer (2010) demonstrates that in addition to wall color, ceiling color also impacts spaciousness perception. Their results show that a brighter ceiling increases experienced spaciousness. Besides color, lighting conditions have also been identified as a factor impacting spaciousness perceptions. Flynn, Spencer, Martyniuk, and Hendrick (1973) investigated the effects of different lighting arrangements on spaciousness scores and found that lighting all four walls of the room creates a more spacious environment than merely lighting the center of the room. This suggests that brighter lighting conditions (i.e., lighting the entire room) induce greater feelings of spaciousness, which is indeed confirmed by experiments with varying room brightness (Durak, Olgonturk, Yener, Guvenc \& Gurcinar, 2007; Manav, 2007). For instance, Durak et al. (2007) varied room brightness and found that brighter conditions were judged as more spacious. Also, Manav (2007) examined the effects of room brightness in an office environment and found that higher levels of room brightness induced more comfort and spaciousness compared to lower levels of room brightness.

Overall, the finding that intangible atmospheric factors are able to create an illusion of a more spacious environment increases opportunities to improve experienced spaciousness of many different environments. Also, from a practical point of view, manipulations related to atmospheric factors are easier to employ than altering architectural or interior design factors, such as adjusting room measurements or removing multiple furniture pieces. It thus seems promising to investigate whether experienced spaciousness is an underlying factor that explains the effect of atmospheric factors such as color and lighting on self-disclosure, making the influence of such complex stimuli yet more comprehensible. 


\section{Online environmental factors influencing spaciousness}

\section{Website design}

Despite the fact that the influence of website design on self-disclosing behavior is still undetermined, findings of consumer research have established that both affect and behavior can be impacted by website design elements (Dailey 2004; Eroglu, Machleit, \& Davis, 2001; Kim, Fiore, \& Lee, 2007; Koo \& Ju, 2010; Mandel \& Johnson, 2002; van Rompay, de Vries, \& van Venrooij, 2010). For instance, Dailey (2004) investigated the effect of website design on feelings of control and psychological reactance and shows that online consumers can experience limited control as a result of using more restrictive navigation cues. In turn such negative feelings as loss of control invoke psychological reactance and cause negative emotions, avoidance behavior and negative website evaluations. The effect of website design on emotions in online consumer research has also been shown by Koo and Ju (2010). Their results show that using distinctive colors and graphics on websites induces pleasure and arousal. Also, results of Kim, Fiore and Lee (2007) show that one can also impact behaviors such as online purchasing and website patronage time by manipulating website design elements such as graphic usage. Similar results were found by Mandel and Johnson (2002) who showed that website design can impact product choice in online store environments.

Although these results do show that website design can impact the online experience and behavior, a lack of knowledge of the underlying processes involved makes it difficult to generalize the reported results and give more general guidelines of how to design your website. Since physical environmental research models are employable or translatable to the online environment, perhaps the impact of underlying processes such as experienced spaciousness is also alike for both environments. There are no results demonstrating that website design influences spaciousness perceptions but results from research on print advertisements do hint that creating a more spacious design can have beneficial effects. It has been shown that white space (i.e., space unfilled with text or graphics) leads to more positive consumer evaluations (Olson, Pracejus, \& O'Guinn, 2012; Pracejus, Olsen, \& O'Guinn, 2006; Strong, 1926) presumably because it enhances spaciousness perceptions.

\section{Situational influences}

The need to keep a certain amount of distance between oneself and others appears to be more urgent in high stress situations (Dosey \& Meisels, 1969; Greene 1977; 
Schiffenbauer \& Schiavo 1976). Dosey and Meisels (1969) studied the effects of stress on interpersonal distance and found that higher stress levels increase one's need for interpersonal distance. In addition, findings by Schiffenbauer and Schiavo (1976) investigated the effects of the tone of interaction and interpersonal distance on liking. Their results showed that during a conversation with a negative tone, participants gave higher likeability scores to the other person at a large interpersonal distance than at a small interpersonal distance. On the other hand, during a positive conversation, participants gave higher likeability scores at a small distance than at a large distance. In a similar vein, Greene (1977) studied the effects of feedback preferred interpersonal distance in a counseling setting. His results indicate that when receiving neutral feedback, participants preferred a larger interpersonal distance in comparison to a smaller interpersonal distance. When receiving positive feedback however, participants preferred a smaller distance to a larger distance. Overall, these results show that people prefer larger interpersonal distances, and perhaps more space in general, when under stress or threat. On the other hand, when stress levels are low and no serious threat is present, smaller interpersonal distances are preferred, indicating that the need for space during such 'positive' circumstances is lower. These results demonstrate that the need for space is thus influenced by the tone of the conversation and that the need for space increases with the communicated threat during conversation. Although speculative, self-disclosure might be affected likewise and may benefit from a more spacious set-up especially during high threat conversations. However, this remains to be examined and therefore situational influences should be taken into consideration when examining environmental influences on self-disclosure. 
In Chapter 2, an overview was presented of previous research on environmental factors and self-disclosure. The following chapter demonstrates that factors on an architectural level and an interior design level are able to influence experienced spaciousness and, in turn, impact self-disclosure intentions. The two studies reported in this chapter examine the effects of room size and desk size on patient readiness to communicate personal information to a physician. Results show that an increase in room size in particular positively influences perceived comfort and intended self-disclosure. Findings of study 3.1 show that spaciousness effects are qualified by patient characteristics. In addition, study 3.2 shows that the affective valence of the conversation influences the found relationship between the environmental manipulations and self-disclosure intentions. 


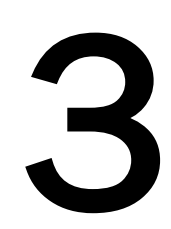

\section{Exploring space in the consultation room: Environmental influences during patient-} physician interaction ${ }^{1}$

1 Okken, V. S., van Rompay, T. J. L., \& Pruyn, A. (2012). Exploring space in the consultation room: Environmental influences during patient-physician interaction. Journal of Health Communication, 17(4), 397-412. 
"Space is what I need, it's what I feed on."

(Peter Gabriel, “Exposure” from the album Peter Gabriel, 1978)

\section{Introduction}

The process of communicating personal information about oneself to another individual is called self-disclosure (Strassberg, Roback, D'Antonio, \& Gabel, 1977). In addition to fostering relationships and affective bonding in general, in healthcare settings self-disclosure is a key factor in the success of a treatment procedure. A lack thereof may prevent a physician from forming an accurate picture of the experienced symptoms and complaints. Conversely, when a patient feels free to disclose information, it facilitates the physician to make an accurate diagnosis and prescribe a patient-tailored treatment (Cegala, Gade, Lenzmeier Broz \& McClure, 2004).

However, from a patient's perspective, disclosing personal information to a physician may be experienced as awkward or shameful and, hence, as something to be avoided. The question thus arises how to facilitate patient selfdisclosure. After reviewing the literature on self-disclosure, we will argue that in addition to characteristics of the communication partners and their behaviors, environmental design may foster self-disclosure by creating the right 'affective atmosphere'. The two studies reported in this paper set out to address this hypothesis.

\section{Self-disclosure}

When considering factors underlying self-disclosure in dyadic interactions, an obvious place to start is with the patient's state of mind or mood. Discussing health issues can be difficult for patients because it increases feelings of vulnerability (Duggan, 2006). In addition, research shows that patients' selfdisclosure varies with their emotional state (Ignatius \& Kokkonen, 2007); a happy, relieved state may more readily prompt information disclosure compared to a worried, anxious state. In addition, dispositional factors such as trait shyness and need for approval have also been found to influence selfdisclosure tendencies (Riley, Cozby, White, \& Kjos, 1983). For instance, Riley et al. (1983) showed that people with high reservedness (a factor akin to shyness) may react with compensatory behaviors (i.e., a refusal to self-disclose) when 
pressured to disclose information, whereas the reverse is true for people with low reservedness.

Besides patient characteristics, also the characteristics of the physician can influence patient self-disclosure. Research shows that gender and age influence the amount of self-disclosure during conversations (Cappella, 1981; Strassberg, Anchor, Gabel, \& Cohen, 1978), with self-disclosure being more frequent for the female sex and with conversation partners of the same age. In addition, familiarity with a doctor is an important factor. In general, communicating personal information is considered to be more appropriate with close friends and relatives than with strangers (Chaikin \& Derlega, 1974; Jourard \& Lasakow, 1958; Mathews, Derlega \& Morrow, 2006; Morton, 1978; Walker, Arnold, MillerDay \& Webb, 2001). Translated to the current context, these findings suggest that self-disclosure is difficult, because physicians are generally strangers and the relationship is strictly professional.

Finally, the unequal distribution of disclosure between both conversation partners can be considered an impediment to self-disclosure. A study by Morton (1978) showed that when communicating with strangers in particular, a high level of reciprocity is conductive to self-disclosure. However, during consults, information flow is one-sided; the patient is expected to self-disclose, without 'receiving' personal information in return. Interestingly, counseling research suggests that 'balancing the scales' may be helpful. Counselors disclosing personal information are not only judged more favorably by their patients (Nilsson, Strassberg \& Bannon, 1979) but also generate more patient disclosure (Roter \& Hall, 1987).

In sum, these findings clarify why self-disclosure is often troublesome for patients. However, their practical implications are limited; fear and worries are salient during consultations, the physician is generally not a close friend, and information flow is by necessity unbalanced. In addition to patient, doctor, and their mutual interaction, the environmental context can be considered a fourth pillar of dyadic conversation.

\section{Self-disclosure and environmental design}

Within the area of healthcare research, a growing body of research provides evidence for the influence of environmental factors on patient experience in general (Dijkstra, Pieterse \& Pruyn, 2006; Ulrich, 1995), and self-disclosure in 
particular (Gifford, 1988; Miwa \& Hanyu, 2006). For instance, research has demonstrated effects of lighting conditions on self-disclosure (Gifford, 1988; Miwa \& Hanyu, 2006). Miwa and Hanyu (2006) showed that dim lighting may positively affect self-disclosure of students in a counseling setting. However, findings reported by Gifford (1988), studying self-disclosure amongst students, showed the reverse effect (i.e., bright lighting yielded more self-disclosure), perhaps suggesting that effects of environmental factors on self-disclosure vary depending on the communication partner (i.e., counselor or fellow-student).

Although these studies provide evidence for the influence of isolated factors in healthcare settings, understanding of how more spatial, holistic influences affect patient behaviors remains limited. This lack of attention is surprising since spatial features may well be of primary importance to the patients' first impression on entering a physician's office. That is to say, before any focused processing of isolated design features, one usually develops an experiential feel for the space that one is in. A small room, for example, may evoke a sense of constraint whereas a large room may trigger feelings of freedom and spaciousness (Meyers-Levy \& Zhu, 2007).

A recent study by Levav and Zhu (2009) demonstrated the importance of store spaciousness and showed that consumers may seek ways to counteract confinement induced by aisle narrowness by making more varied product choices. They suggested that consumers wanted to compensate reductions in spatial freedom by creating more varied product choices indicative of psychological freedom. In line with these findings, Sundstrom (1975) showed that limited space may induce crowding perceptions, which in turn may decrease communicative behaviors. What these findings imply is that limited physical space inspires perceptions of limited psychological space, and that this negative feeling of 'restraint' causes reactance, transpiring in a refusal to comply with explicit or implicit requests (e.g., refusing to disclose information when expected to do so). This explanation is in line with embodiment research showing that spatial (environmental) properties may shape people's reasoning about abstract, mental concepts (cf. IJzerman \& Semin, 2010; Williams \& Bargh, 2008).

Based on these notions, we hypothesize that a decrease in room size induces (negatively charged) feelings of confinement, in turn lowering perceived comfort and self-disclosure. An increase in room size, on the other hand, is expected to generate (positively charged) feelings of freedom and spaciousness, facilitating self-disclosure and generating more perceived comfort. Hence: 
$H 1 A$ : There is a positive relationship between room size and perceived comfort, which is mediated by perceived spaciousness.

H1B: There is a positive relationship between room size and intended selfdisclosure, which is mediated by perceived spaciousness.

Interior design features may also affect perceived spaciousness. A study of particular relevance addressed the relationship between interpersonal distance and self-disclosure (Jourard \& Friedman, 1970). Results showed that a decrease in distance caused a decrease in self-disclosure, most notably in the case of marked status differences. Along similar lines, Albert and Dabbs (1970) examined the effect of persuasive messages at different interpersonal distances. Their results demonstrate that when the interpersonal distance decreased, reactance increased and participants were less likely to accept persuasive messages.

Based on these findings, we expect that a reduction in interpersonal distance between physician and patient increases (negatively charged) feeling of confinement, thereby reducing perceived comfort and self-disclosure intentions. Conversely, a larger distance is expected to trigger (positively charged) feelings of freedom and spaciousness, in turn leading to increased self-disclosure and positively affecting perceived comfort. Hence:

H2A: There is a positive relationship between desk size and perceived comfort, which is mediated by perceived spaciousness.

H2B: There is a positive relationship between desk size and intended selfdisclosure, which is mediated by perceived spaciousness.

Obviously, personal differences can also influence the general tendency to disclose personal information, with shy or reserved people showing an overall lower willingness to disclose information (Riley, Cozby, White, \& Kjos, 1983). Hence, for explorative purposes, a measure indicative of reservedness was incorporated.

To test the hypotheses, interpersonal distance and room size were manipulated in a photograph of a consultation room, resulting in a 2 (interpersonal distance: small versus large) $\times 2$ (room size: small versus large) $\times 2$ (trait reservedness: low versus high) between-subjects design.

The use of photographic material in environmental research has been shown to accurately simulate real environments (Bateson \& Hui, 1992; Hendrick, Martyniuk, Spencer \& Flynn, 1977; Stamps, 1990). For instance, a meta- 
analysis by Stamps (1990) of research comprising both measurements obtained in situ and measurements obtained through photographic material revealed a 0.86 correlation.

\section{Study 3.1}

\section{Method}

Participants and procedure

A total of 110 participants (48 male and 62 female; mean age 53.11 years, SD 16.02) participated in the experiment. Participants were recruited by approaching passers-by in the center of a large town in the Netherlands with the request to take part in a study on their impression of healthcare environments. No rewards were given for participation. Participants were informed in the introduction that the purpose of the study was to extend knowledge about patients' judgments of consultation rooms. Next, they were presented with the following scenario:

"Imagine that you recently visited a general practitioner after experiencing palpitations. Following this consult, an appointment was scheduled with a cardiologist at the local hospital. Today is your first appointment with cardiologist Ben Jansen. During this session, you are asked to reveal information about yourself and your specific complaints."

The participants were subsequently presented with an image of one of the consultation rooms and asked to imagine themselves in the situation depicted. Then a questionnaire was presented, comprising the dependent variables and the reservedness scale. On completion, participants were thanked for their cooperation and were dismissed.

\section{Stimulus material}

In order to manipulate perceived spaciousness, interpersonal distance and room size were varied. For the construction of the four different variants, one template of a consultation room was digitally modified (see Figure 3.1). Room size was manipulated by adding a corner behind the table to create the illusion of a smaller room. In the image of the large room, no corners were visible, thus creating the illusion of a larger room. 

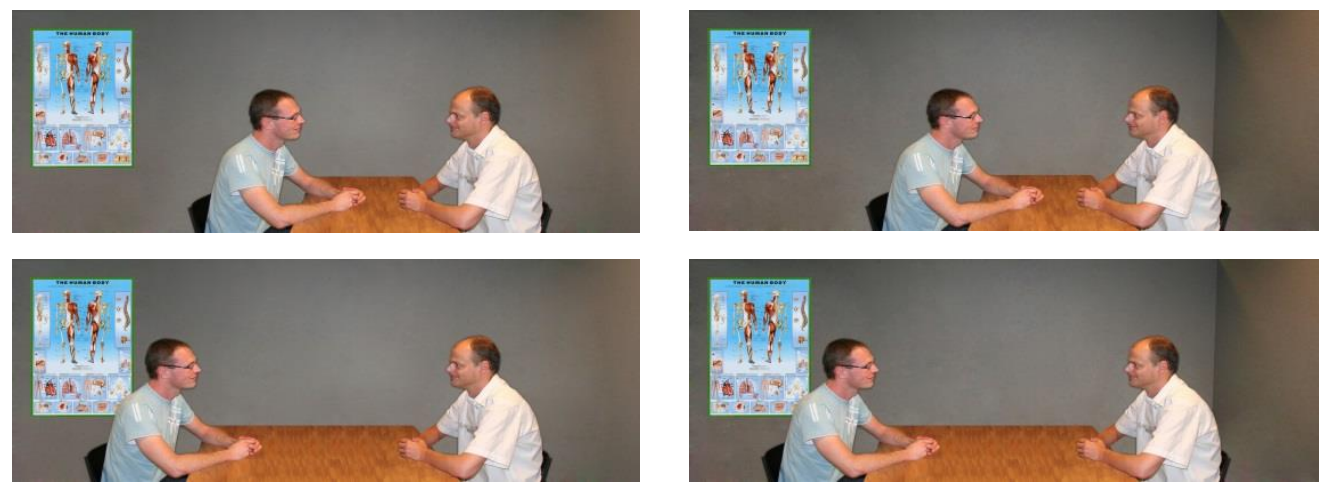

Figure 3.1. The four pictures of the different consultation rooms. Desk size is small for the upper two pictures, and large for the lower two pictures. Room size is small for the pictures on the right, and large for the pictures on the left.

In order to manipulate interpersonal distance, desk size was varied. A pilot study was conducted in which 12 participants were shown a series of desk sizes and asked to indicate what they considered to be a realistic small and a realistic large seating distance for patient-physician interaction during a consult. Based on these results, the desk sizes were set at $80 \mathrm{~cm}$ for the small distance and at $130 \mathrm{~cm}$ for the large distance.

\section{Measures}

Responses to all scales were recorded on seven-point rating scales.

Perceived spaciousness. Perceived spaciousness was measured using the items: "I would feel constricted in this room" (reverse coded), "I would feel confined in this room" (reverse coded), "I would have sufficient freedom of movement in this room" and "I would easily feel suffocated in this room" (reverse coded) $(M=4.19, S D=1.77, \alpha=.96)$.

Perceived comfort. Perceived comfort was measured using the items: "In this room I would feel at ease", "In this room I would feel unhappy" (reverse coded), "I would feel uncomfortable in this room" (reverse coded), and "This room would give me a pleasant feeling" $(M=3.59, S D=1.71, \alpha=.95)$.

Intended self-disclosure. Intended self-disclosure was measured using the items: "I would feel inhibited from speaking in this room" (reverse coded), "In this room I would feel able to speak freely", "I would feel uncomfortable to share personal information in this room" (reverse coded), and "It would be hard for me to talk about myself in this room" (reverse coded) $(M=4.51, S D=1.45, \alpha=$ $.92)$. 
Trait reservedness. Participants' dispositional reservedness was measured using a scale comprising 23 items $(M=3.28, S D=0.79, \alpha=.90$; based on the defensiveness subscale of the Dutch version of the Minnesota Multiphasic Personality Inventory-2, by Derksen, de Mey, Sloore \& Hellenbosch, 1993), measuring the extent to which participants are inclined to engage in social interactions with items such as "I am a very social person" and "I find it easy to talk with others in an easy and pleasant manner". Responses were recorded on seven-point rating scales, with higher scores indicating a higher level of reservedness in social interaction.

\section{Results}

Results were analyzed for gender and age, but since none proved significant there will be no further discussion of these variables. In the following, ANOVAs were used with desk size, room size and trait reservedness as independent variables and perceived spaciousness, intended self-disclosure, and perceived comfort as dependent variables. To analyze the reservedness scores, a median split was used $(M d n=3.30)$.

\section{Perceived spaciousness}

Desk size had an effect on perceived spaciousness $(F(1,106)=68.23, p<$ .001 , partial $\eta^{2}=.41$ ). In line with predictions, perceived spaciousness scores for the room with the large desk were higher $(M=5.20, S D=.18)$ than for the room with the small desk $(M=3.08, S D=.19)$, indicating that participants felt less restricted in the large desk size condition compared to the small desk size condition.

Room size also had an effect on perceived spaciousness $(F(1,106)=15.67, p$ $<.001$, partial $\left.\eta^{2}=.14\right)$. As expected, ratings for the large room $(M=4.65, S D=$ .18 ) were higher than ratings for the small room $(M=3.63, S D=.18)$, indicating that participants experienced more spaciousness in the large room than in the small room.

An interaction was obtained between desk size and room size $(F(1,106)=$ 4.38, $p=.039$, partial $\left.\eta^{2}=.04\right)$. In the small desk situation, the difference in mean perceived spaciousness scores for room size was significant, with respondents judging the large room as more spacious than the small room $(M=$ $3.86, S D=.26$ vs. $M=2.30, S D=.27 ; F(1,99)=17.46, p<.001)$. In the large desk situation, the difference in spaciousness scores was not significant (small desk: $M=4.96, S D=.25$ vs. large desk: $M=5.44, S D=.25 ; F(1,99)=1.83, p$ $=.180)$. 
An additional interaction was obtained between room size and trait reservedness $\left(F(1,106)=4.89, p=.029\right.$, partial $\left.\eta^{2}=.05\right)$. Respondents with low reservedness rated the large room higher on perceived spaciousness than the small room $(M=4.83, S D=.26$ vs. $M=3.24$, $S D=.25 ; F(1,99)=19.92, p$ $<.001$ ). Respondents with high reservedness rated both rooms alike (small room: $M=4.02, S D=.27$ vs. large room $M=4.47, S D=.25 ; F(1,99)=1.46, p$ $=.230)$.

\section{Intended self-disclosure}

Desk size had an effect on intended self-disclosure $(F(1,106)=24.25, p<$ .001 , partial $\eta^{2}=.20$ ). As predicted, the large desk yielded higher scores for intended self-disclosure $(M=5.09, S D=.17)$ in comparison to the small desk $(M=3.89, S D=.18)$. Room size also had an effect on intended self-disclosure $\left(F(1,106)=5.81, p=.018\right.$, partial $\left.\eta^{2}=.06\right)$. The large room triggered more intended self-disclosure $(M=4.78, S D=.17)$ than the small room $(M=4.18$, $S D=.18)$.

An interaction was obtained between room size and trait reservedness ( $F(1$, $106)=5.60, p=.020$, partial $\left.\eta^{2}=.05\right)$. Respondents with low reservedness showed a higher tendency to disclose information in the large room, compared to the small room $(M=5.23, S D=.26$ vs. $M=4.05, S D=.24 ; F(1,99)=11.94$, $p=.001)$. For respondents with high reservedness, this difference was not significant (small room $M=4.32, S D=.25$ vs. large room $M=4.33, S D=.24 ; F$ $<1, n s)$.

\section{Perceived comfort}

As predicted, analyses revealed a main effect of desk size on comfort ( $F$ ( 1 , 106) $=60.84, p<.001$, partial $\eta^{2}=.38$ ), indicating that comfort was higher in the large desk condition $(M=4.53, S D=.17)$ compared to the small desk condition $(M=2.56, S D=.18)$. In line with the results for perceived spaciousness and intended self-disclosure, the main effect for room size was significant $\left(F(1,106)=10.30, p=.002\right.$, partial $\left.\eta^{2}=.09\right)$, showing that participants felt more at ease in the large room $(M=3.95, S D=.18)$ than in the small room $(M=3.14, S D=.18)$.

In addition, an interaction was obtained between desk size and room size ( $F$ (1, $106)=5.69, p=.019$, partial $\left.\eta^{2}=.05\right)$. When desk size was small, there was a significant difference in comfort scores for the different room sizes $(M=1.85$, $S D=.26$ vs. $M=3.27, S D=.25 ; F(1,99)=14.93, p<.001)$, indicating that participants experienced the large room as more pleasing than the small room. In the large desk size condition, this difference did not reach significance (small room $M=4.42, S D=.25$ vs. large room $M=4.63, S D=.25 ; F<1$, ns). 
Finally, the interaction between room size and reservedness was significant ( $F$ $(1,106)=6.90, p=.010$, partial $\left.\eta^{2}=.07\right)$. Respondents low in reservedness gave higher comfort scores to the large room, as compared to the small room $(M=4.10, S D=.25$ vs. $M=2.63, S D=.24 ; F(1,99)=17.82, p<.001)$. Respondents high in reservedness rated the both rooms alike (small room $M=$ $3.65, S D=.27$ vs. $M=3.90, S D=.25 ; F<1, n s)$.

\section{Mediation analyses}

Mediation analyses, following the procedure outlined by Baron and Kenny (1986), were conducted in order to test whether the effects of room size and desk size on the dependent variables (i.e., perceived comfort and intended selfdisclosure) are indeed mediated by perceived spaciousness (see Figure 3.2). All reported regression coefficients are standardized.

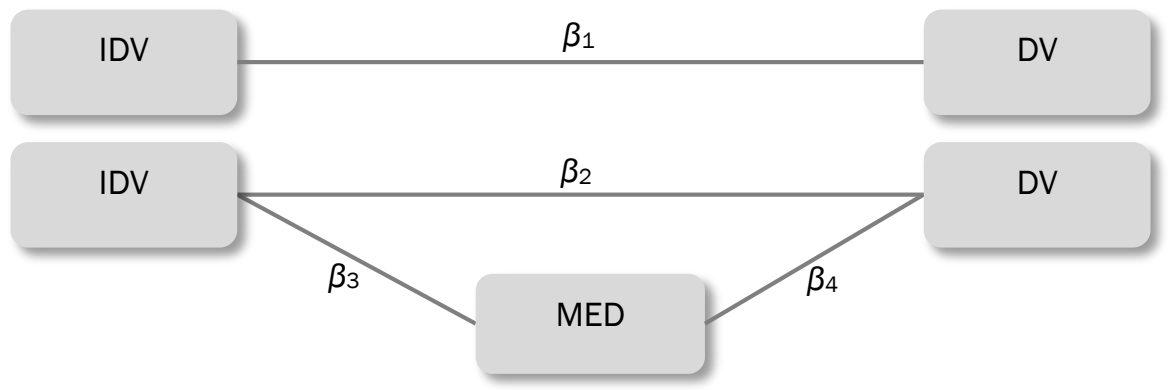

Figure 3.2. Mediation model, with room size or desk size as the independent variable (IDV), perceived spaciousness as the mediator (MED), and perceived comfort or intended self-disclosure as dependent variable (DV).

First we tested whether room size influences perceived comfort via perceived spaciousness (H1A). Analyses showed that the effect of room size on perceived comfort is significant ( $\beta=.24, p=.014$ ). Likewise, the effect of room size on perceived spaciousness (the mediator) reached significance $(\beta=.28, p=.004)$, as did the effect of perceived spaciousness (the mediator) on perceived comfort (the dependent variable) $(\beta=.94, p<.001)$. When both room size and perceived spaciousness were included in the model, the effect of room size on perceived comfort became non-significant $(\beta=-.02, p=.484)$, whereas the effect of perceived spaciousness on perceived comfort remained significant $(\beta=$ $.95, p<.001$ ). In addition, results of a Sobel test showed the indirect effect (of room size on comfort via perceived spaciousness) to be significant (Sobel $z=$ $2.93, p=.003)$. This indicates a mediating effect of perceived spaciousness on the relationship between room size and perceived comfort, and confirms hypothesis $1 \mathrm{~A}$. 


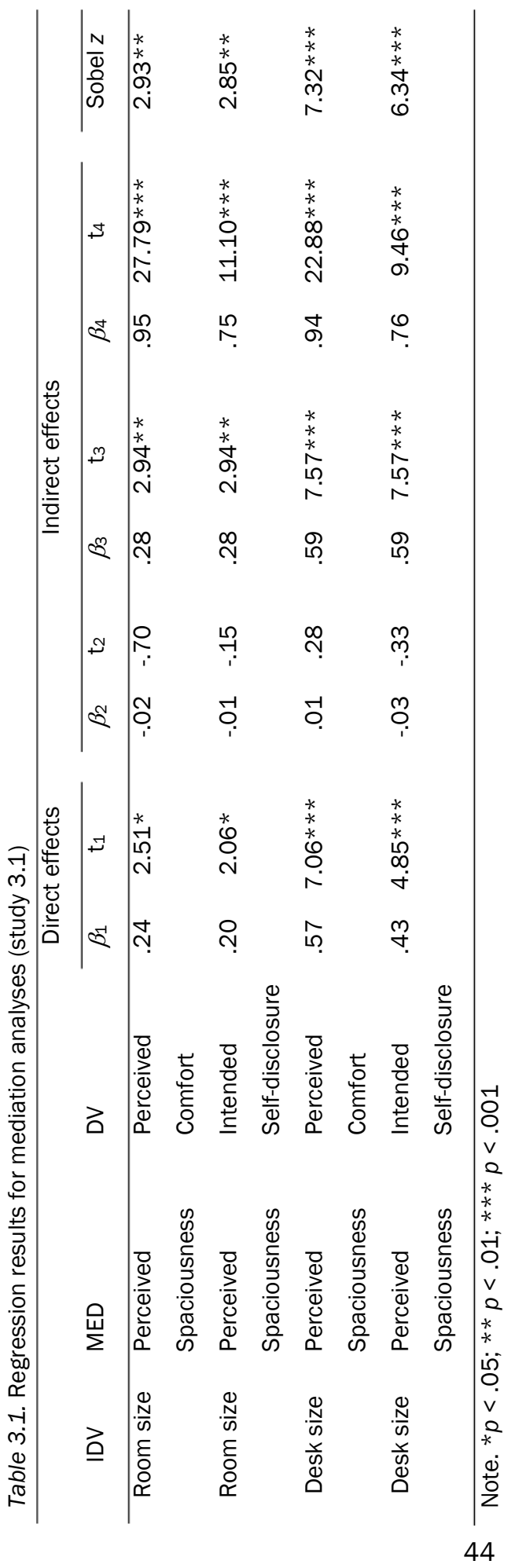


In order to test the mediation hypotheses $1 \mathrm{~B}, 2 \mathrm{~A}$ and $2 \mathrm{~B}$, similar analyses were conducted (see Table 3.1). Results confirm the predicted mediations and the role of perceived spaciousness in explaining effects of both desk size and room size on perceived comfort and intended self-disclosure.

\section{Discussion}

The results confirm our expectations concerning the influence of perceived spaciousness, thereby advancing knowledge about the ways in which interior and architectural factors impact patient experience. As such, perceived spaciousness can be considered a function of interior design (i.e., desk size or seating distance) and architectural design (room size). Most importantly, the reported results show that perceived spaciousness affects both perceived comfort and intended selfdisclosure. This finding is in line with findings reported in previous research (Jourard \& Friedman, 1970). Interestingly, individual differences were found to interact with architectural design. Participants with low reservedness were shown to be more sensitive to the architectural design of the room. These results do not justify any definite conclusions, but one could argue that people high in reservedness are more likely to feel threatened by social interactions and thus focus all their attention on the possible threat (i.e., the physician) thereby neglecting their surroundings.Although the reported results suggest that an increase in distance promotes self-disclosure, previous research showed that a reduction in interpersonal distance may also stimulate social interactions (Albas, 1991; Sommer, 1959). Even though care was taken to create a neutral scenario, perhaps the conversation was negatively interpreted. This might contribute to an overall negative feeling, increasing the need for spaciousness. Arguably, a more positively framed scenario might decrease the need for spaciousness, as suggested by findings by Schiffenbauer and Schiavo (1976) addressing the combined effect of interpersonal distance and the tone of conversation on judgments of others. Their results indicate that a small interpersonal distance promotes likability when the tone of the conversation is positive. When the tone of the conversation is negative, larger interpersonal distances are preferable. These findings suggest that in threatening situations, patients prefer more space as a means to (literally) keep the threat at a distance. In non-threatening situations, a smaller distance might be preferred because it enables more intimate and personal interaction. Based on these findings, we propose that the effects obtained in study 3.1 are qualified by the affective valence of the scenario. Hence:

H3A: The positive effect of room size on perceived comfort and intended selfdisclosure is moderated by the affective valence of the conversation. Specifically, these effects are more pronounced when the scenario is negatively, rather than positively, framed. 
H3B: The positive effect of desk size on perceived comfort and intended selfdisclosure is moderated by the affective valence of the conversation. Specifically, these effects are more pronounced when the scenario is negatively, rather than positively, framed.

In the second study, we thus manipulated the affective valence of the conversation in addition to room size and interpersonal distance. This resulted in a 2 (interpersonal distance: small versus large) $\times 2$ (room size: small versus large) $\times 2$ (affective valence of the conversation: positive versus negative) between-subjects design.

\section{Study 3.2}

\section{Method}

A total of 188 participants (66 male and 122 female; mean age 22.0 years; $S D=$ 3.27) participated in the second study. Participants were recruited by approaching passers-by on the campus of a Dutch university with the request participate in a short study on their impression of healthcare environments. All respondents were students enrolled in various (under)graduate programs at the university. The introduction to the experiment was identical to study 3.1. Next, either a positive scenario (a conversation with a doctor following a 'nothing to worry about' checkup was presented) or a negative scenario (a conversation following a more troublesome checkup) was presented. The scenario presented to the participants (translated from Dutch; manipulations displayed in bold typeface; wording used in the negative condition between brackets) stated:

"About eight weeks ago you visited your general practitioner because you experienced a skin irritation on your abdomen and back. You were referred to a dermatologist at the local hospital. In the following period, several medical tests took place and the dermatologist provided you with a zinc ointment to rub on the irritated parts of your body. The treatment appears (not) to work because you experience less itching (more itching) and the irritation has almost disappeared (seems to be getting worse). Last week you had a telephone consultation with your dermatologist. He informed you that the test results of the latest test are in and that it appears to be nothing serious (and that the results are inconclusive). Today you have an appointment with the dermatologist to discuss the test results and your experiences and have an additional check-up. You feel relieved (worried) because the itching and irritation pose no serious threat (the source of your 
complaints is unclear), and have almost disappeared (that it is hard to assess the seriousness of the health threat involved)."

The participants were then presented with an image of one of the consultation rooms (identical to study 3.1; see Figure 3.1) and asked to imagine themselves in the situation depicted. Next, the questionnaire - similar to study 3.1 - was presented, comprising the dependent variables perceived spaciousness $(M=3.45$, $S D=1.13, \alpha=.79)$, perceived comfort $(M=2.76, S D=1.06, \alpha=.76)$ and intended self-disclosure $(M=3.79, S D=1.27, \alpha=.87)$. On completion, participants were thanked for their cooperation and were dismissed.

\section{Results}

Results were analyzed for gender and age, but since none proved significant they will not be discussed further. Results were analyzed using ANOVAs with desk size, room size and affective valence of the conversation as the independent variables and perceived spaciousness, intended self-disclosure, and perceived comfort as the dependent variables.

\section{Perceived spaciousness}

Desk size had an effect on perceived spaciousness $(F(1,182)=4.68, p<.05$, partial $\left.\eta^{2}=.03\right)$. In line with the previous study, perceived spaciousness scores for the room with the large desk were higher $(M=3.63, S D=.11)$ than for the room with the small desk $(M=3.29, S D=.11)$, indicating that participants felt less restricted in the large desk size condition compared to the small desk size condition.

Room size also had an effect on perceived spaciousness $(F(1,182)=17.01, p<$ .001 , partial $\left.\eta^{2}=.09\right)$. As expected, the large room was rated higher on perceived spaciousness $(M=3.78, S D=.11)$ than the small room $(M=3.13, S D=.12)$, indicating that participants felt less restricted in the large room than in the small room. No further interactions were obtained.

Intended self-disclosure

Analyses yielded a significant main effect of room size on intended self-disclosure $(F$ $(1,182)=8.64, p<.01$, partial $\left.\eta^{2}=.05\right)$, indicating that the large room yielded higher scores for intended self-disclosure $(M=4.05, S D=.13)$ compared to the small room $(M=3.53, S D=.13)$. No significant main effect was obtained for desk size $(F<1, n s)$.

A three-way interaction was obtained between desk size, room size and the affective valence of the conversation $\left(F(1,182)=7.36, p<.01\right.$, partial $\left.\eta^{2}=.04\right)$. 
For the positively framed scenario, a significant effect was found in the large room condition (see Figure 3.3); the small desk yielded more intended self-disclosure than the large desk $(M=4.50, S D=.25$ vs. $M=3.48, S D=.27 ; F(1,182)=7.97$, $p=.005)$. In the small room condition, the difference between desk sizes did not reach significance. For the negatively framed scenario, no significant effects for desk size were obtained. Thus although hypotheses $3 \mathrm{~A}$ and $3 \mathrm{~B}$ are not confirmed, these findings do show that the affective valence of the conversation may qualify effects of room size and desk size.

\section{Perceived comfort}

Room size was found to have a significant effect on perceived comfort $(F(1,182)=$ $10.44, p=.001$, partial $\left.\eta^{2}=.05\right)$, indicating that participants rated the large room higher on comfort $(M=3.01, S D=.11)$ than the small room $(M=2.53, S D=.11)^{2}$. As in study 3.1, mediation analyses were conducted to test whether the effects of room size on both perceived comfort and intended self-disclosure were mediated by perceived spaciousness. Results showed this to be the case (perceived comfort: Sobel $z=3.85, p<.001$; intended self-disclosure: Sobel $z=3.71, p<.001$ ). As the main effects of desk size on perceived comfort and intended self-disclosure were not significant, no mediation analyses were conducted for the effects of desk size.

\section{Discussion}

The results of the second study are largely in line with the findings from study 1 by replicating the effects of room size on intended self-disclosure. However, the main effects of desk size were not significant in study 3.2. In addition, the affective valence did not qualify the effects of room size and desk size in the negative scenario. Nonetheless, affective valence did moderate the effects of room size and desk size in the positive scenario. More specifically, in the large room participants showed higher intentions to self-disclose in the small desk condition in comparison to the large desk condition.

\footnotetext{
2 As in study 3.1, mediation analyses were conducted to test whether the effects of room size on both perceived comfort and intended self-disclosure were mediated by perceived spaciousness. Results showed this to be the case (perceived comfort: Sobel $z=3.85, p<$ .001 ; intended self-disclosure: Sobel $z=3.71, p<.001$ ). As the main effects of desk size on perceived comfort and intended self-disclosure were not significant, no mediation analyses were conducted for the effects of desk size.
} 


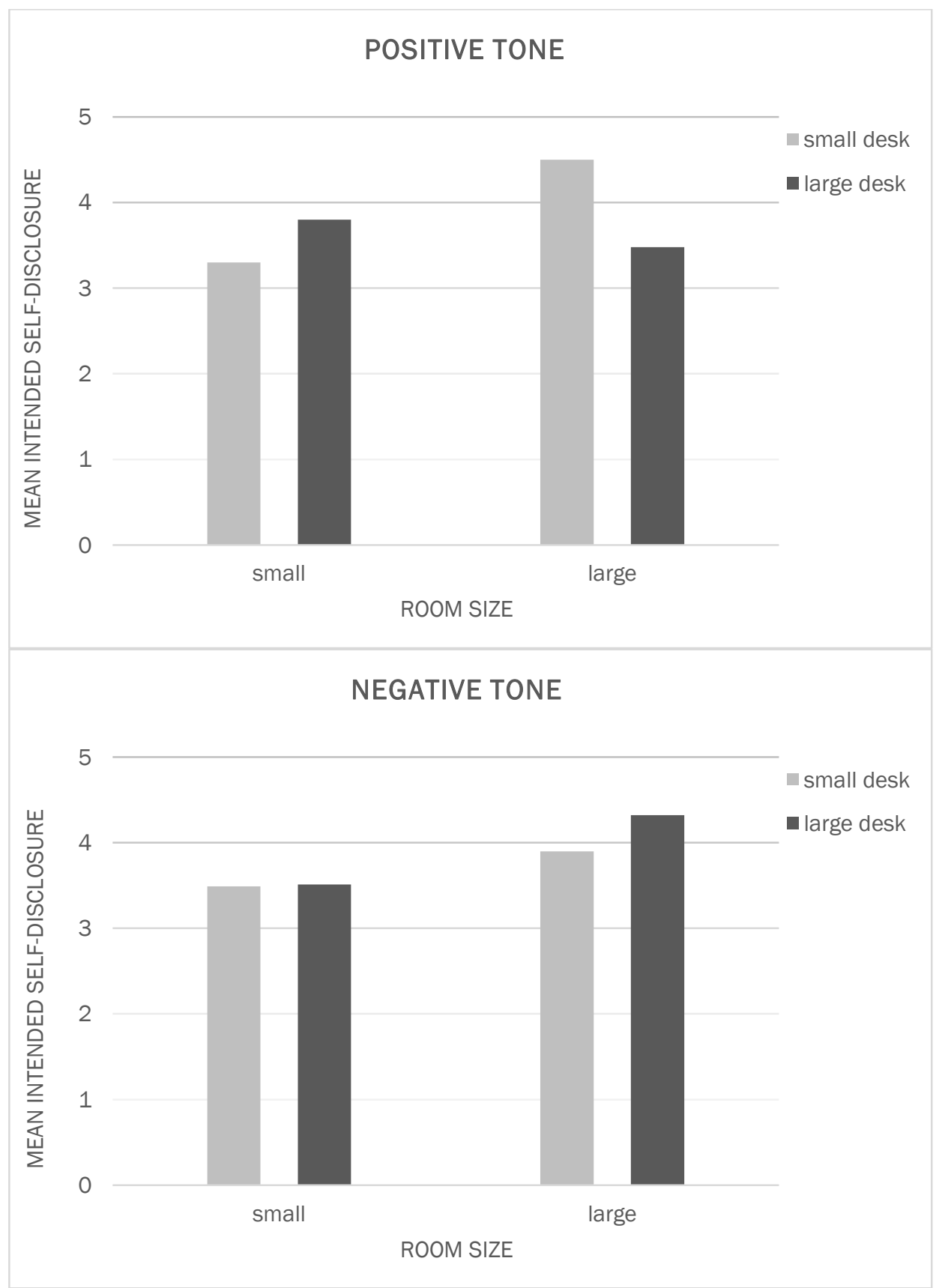

Figure 3.3. Three-way interaction between the affective valence of the conversation, room size, and desk size for intended self-disclosure, with the upper graph showing the positive affective valence and the lower graph showing the negative affective valence. 


\section{General discussion}

The results presented stress the importance of attending to spatial features of consultation rooms in patient-doctor interactions. The findings of the two studies indicate a general preference for more spaciousness on an architectural level (i.e., increased room size). However, the effects of desk size are not consistent across the two studies (they did not reach significance in study 3.2). Nevertheless, the effect of desk size did transpire in the obtained interaction in study 3.2. More specifically, the results show that patients value proximity or decreased distance when the situation is stress-free and the room is large. As for the non-significant effects of room size and desk size in the negative scenario, perhaps participants paid less attention to the environmental factors as a result of the threat presented in the scenario. Likewise, findings from study 3.1 showed that participants high in social anxiety (i.e., high reservedness) are also less sensitive to environmental factors. These overall findings indicate that anxiety (either resulting from personality as in study 3.1 or from the tone of the conversation as in study 3.2) may lead participants to focus more strongly on the content of the message rather than on the environment.

In accounting for the effects observed, of particular interest are the results of Schiffenbauer and Schiavo (1976) discussed earlier. Along similar lines, Greene (1977), studying interpersonal distance in a counseling setting as a function of feedback type, showed that when feedback was positive, a smaller distance invoked more positive affect and therapy loyalty in comparison to a greater distance. When feedback was neutral the situation was reversed and a greater distance was preferred - a finding in line with the results from study 3.1. This suggests that in non-threatening situations smaller distances might be preferred because they enable more personal interaction, a finding in line with studies suggesting that more personal or intimate environments promote self-disclosure (e.g., Miwa \& Hanyu, 2006).

With respect to managerial implications, effects and implications of interior design and atmospheric factors can be relatively easily incorporated in design guidelines for existing buildings. For instance, decisions regarding interpersonal distance and positioning of furniture are easily incorporated. Considering the large variation in situational contexts of conversations taking place in consultation rooms, a flexible environment that can be tailored to patients' needs is arguably preferable (i.e., extendible desks), to the extent that it allows physicians to 'adjust' perceived room spaciousness. It is an open question, however, to what extent attributions about the physician differ when patients feel that the distance between patient and physician is intentionally created by the physician. For instance, a physician intentionally 
moving his chair further away from the table may come across as distant or indifferent.

Field studies investigating actual patient behaviors in real environments representative of actual consultation rooms are necessary to obtain further insights into the workings of environmental factors. And, although our results indicate that relative differences in room size and desk size affect patient outcomes, they do not warrant any conclusions regarding absolute room size (and desk size). Nonetheless, it can be expected that extreme measurements (either very small or very large) are likely to result in negative patient experiences.

In follow-up studies, another factor that can be taken into consideration is the nonverbal behavior displayed by both physician and patient. Research suggests that people resort to nonverbal behaviors (e.g., avoiding or establishing eye contact, or turning one's body away from or towards the conversation partner) to maintain an 'intimacy equilibrium' during dyadic conversations (Argyle \& Dean, 1965; Patterson, 1973). Thus, in addition to verbal communication, nonverbal communication can also be used as a means to create and maintain the 'right' level of intimacy. Another factor that should be included in future studies is physician gender. Although in the experiments reported, no differences were observed between male and female participants, physician and patient gender were not explicitly taken into account as an experimental factor.

Awaiting follow-up research, the results presented meanwhile demonstrate the importance of controlled research addressing a neglected but potentially important and powerful dimension of healthcare environments. 
Chapter 3 demonstrated that both room size and desk size impact experienced spaciousness and, in turn, self-disclosure intentions during experiments using pictures of a simulated consult with a general practitioner. The findings show that increases in physical space facilitate self-disclosure and trigger a more positive affective experience. Chapter 4 investigates whether these effects can also be found when using an actual environmental setup, as opposed to a depicted scene, hence adding the possibility to measure actual self-disclosing behavior. The study reported investigates the effect of spaciousness impressions, again by manipulating room size and desk size, during interviews on intimate lifestyle-related topics. The results show that room size in particular affects self-disclosing behavior with increases in architectural space positively affecting self-disclosure. Interestingly, the effects obtained varied considerably across the different topics. Furthermore, nonverbal measures revealed that posture readjustments and refrains from establishing eye contact are used to counteract lack of sufficient space. 


\section{4}

\section{Room to move: On spatial constraints and self-disclosure during intimate conversations ${ }^{3}$}

3 Okken, V. S., van Rompay, T. J. L., \& Pruyn, A. (2013). Room to move: On spatial constraints and self-disclosure during intimate conversations. Environment \& Behavior, 45(6), 737-760. 
"Her apartment felt particularly small. It had a way of expending or contracting, depending on how the day went. Today it struck her as ludicrous, these little rooms in which she found herself living."

(Michael Cunningham, Specimen Days, 2005)

\section{Introduction}

Sharing personal information is essential to the development and maintenance of personal and professional relationships. Whether it involves communication between friends, colleagues or strangers, self-disclosure makes relationships not only interesting and engaging, it also provides communication partners with the necessary information required to respond to each other's needs. As such, selfdisclosure (i.e., the process of communicating personal information to another person; Chelune, 1975; Omarzu, 2000; Strassberg, Roback, D’Antonio, \& Gabel, 1977 ) is likewise essential to communication processes taking place in many service settings in which input from clients or patients is crucial to service providers, as is the case in counseling and healthcare settings (Cohen \& Schwartz, 1997; Hinson \& Swanson, 1993). In these settings, clients or patients may be asked to provide information on lifestyle, medical history and physical or psychological problems; information that enables caregivers to make an accurate diagnosis of the problems involved (Cegala, Gade, Lenzmeier Broz \& McClure, 2004). Although in such cases, patients usually take the initiative to seek help, many nonetheless find it embarrassing or troublesome to share their problems with a 'stranger', turning self-disclosure into a negatively laden experience.

Research shows that self-disclosure varies with person and conversation-related factors. For instance, it has been shown that the discloser's state of mind influences the likelihood of sharing personal information (Cunningham, Steinberg \& Grev, 1980; Forgas, 2011; Ignatius \& Kokkonen, 2007), with a positive mood increasing the disclosure tendency. In addition, recipient characteristics such as gender, age, and status influence self-disclosure, with disclosure more easily taking place towards women and between people of the same age and status (Chaikin \& Derlega, 1974; Cappella, 1981; Collins \& Miller, 1994), although with respect to the former, results of a meta-analysis indicate that sex differences in self-disclosure are smaller than expected (Dindia \& Allen, 1992). As for interpersonal feelings or emotions: trust, liking, and familiarity positively influence disclosure (Chaikin \& Derlega, 1974; Collins \& Miller, 1994; Mathews, Derlega \& Morrow, 2006; Rotter, 1980). 
Apart from varying with mood, relationship type and person characteristics, conversation characteristics may also facilitate or hinder self-disclosure. Generally, self-disclosure is more troublesome during conversations reflecting personal, intimate and, hence, potentially embarrassing topics such as personal fears, emotions, self-incriminating information and sexual behaviors (Altman \& Taylor, 1973; Howell \& Conway, 1990; Joinson, Paine, Buchanan \& Reips, 2008). For instance, Joinson et al. (2008) showed that self-disclosure decreases with increasing sensitivity of the personal information involved. Finally, self-disclosure may increase in order to reciprocate disclosure by the conversation partner, suggesting that individuals may seek equilibrium in terms of disclosure towards one another (Cozby, 1973; Ignatius \& Kokkonen, 2007; Morton, 1978).

In addition to such well-established influences, the environment in which conversations take place can also facilitate or hinder disclosure. For instance, various studies indicate that creating more comfortable or pleasurable environments (e.g., through lighting) stimulates self-disclosure, arguably because such environments make individuals feel comfortable and at ease (Chaikin, Derlega \& Miller, 1976; Gifford, 1988; Miwa \& Hanyu, 2006). Of particular relevance to current undertaking, another line of self-disclosure research hints at the importance of experienced spaciousness for stimulating self-disclosure (Okken, Van Rompay \& Pruyn, 2012; Sundstrom, 1975). For instance, Okken et al. (2012) showed that increases in room size positively affect self-disclosure intentions and the affective experience. Although research shows that self-reported self-disclosure tendencies can predict actual disclosing behavior (Halpern, 1977; Kahn, Lamb, Champion, Eberle \& Schoen, 2002), it is an open question whether actual (as opposed to intended) self-disclosure is sensitive to spatial constraints. Furthermore, effects of spatial factors may vary across topics with some topics 'requiring' more space to unfold than others. Therefore, this study investigates effects of spaciousness impressions on actual disclosing behaviors and participants' affective experiences during interviews on a variety of lifestyle-related topics.

\section{Spaciousness and self-disclosure}

Long-standing research findings testify to the importance of the physical environment for self-disclosure (Chaikin, Derlega \& Miller, 1976; Cohen \& Schwartz, 1997; Jourard \& Friedman, 1970; Lecomte, Bernstein \& Dumont, 1981; Sundstrom, 1975). For instance, a study by Sundstrom (1975), addressing the effect of room size on stress and self-disclosure, showed that limited space may induce crowding perceptions and as a result may decrease communicative behaviors. Jourard and Friedman (1970) studied the effects of interpersonal 
distance during interviews. Results of their study showed that when the physical distance between experimenter and participant decreases, so does the extent of self-disclosure. These findings indicate that spatial aspects of an environment influence disclosing behavior, and they suggest that spaciousness impressions (i.e., perceptions of feeling free or confined) may be triggered by environmental factors pertaining to positioning of furniture and inhabitants (e.g., interpersonal distance), and by architectural dimensions (i.e., room size). More recently, Okken, van Rompay and Pruyn (2012) studied the effects of both room size and interpersonal distance on self-disclosure intentions and affective experiences in experiments involving pictures of a simulated patient consult with a general practitioner. Their results show that both room size and desk size influence self-disclosure intentions and positive affect, and that these relationships are mediated by spaciousness perceptions. These findings suggest that increases in physical space may generate psychological space, in turn facilitating self-disclosure and triggering a more positive affective experience.

In line with these findings, research in environmental psychology (see Stamps, 2011) and consumer research (Levav \& Zhu, 2009; Meyers-Levy \& Zhu, 2007) also testifies to the beneficial effects of experienced spaciousness. For instance, Meyers-Levy and Zhu (2007) showed that a high, as opposed to a low, ceiling (also increasing spaciousness) may activate feelings of freedom, subsequently generating more creative strategies in a problem-solving task. Similarly, Levav and Zhu (2009) investigated the effects of spaciousness in store environments. They showed that narrow shopping aisles activated feelings of confinement, and that these negative feelings are counteracted by making more varied product choices (e.g., in western societies viewed as an act / expression of freedom). These combined findings suggest that restrained physical space may indeed invoke feelings of limited psychological space (cf. Okken et al, 2012), a proposition also in line with recent embodiment research showing that spatial properties influence people's reasoning about abstract, mental concepts (IJzerman \& Semin, 2010). A central assumption underlying current research holds that spatial constraints in counseling or health settings likewise generate a freedom-seeking tendency and, hence, a refusal to comply with behavioral norms (e.g., 'reactance'). In the context of a consult or interview, such a refusal should transpire in a lowered willingness to disclose personal information.

In sum, we argue that both architectural and interior design-related interventions (i.e., increases in room size or volume and interpersonal distance respectively) may increase experienced spaciousness and feelings of freedom, in turn leading to more self-disclosure. Conversely, limiting spaciousness may elicit feelings of restraint, in turn leading to a refusal to disclose information. Hence: 
H1a: Increases in room size induce feelings of spaciousness, thereby generating more self-disclosure.

$H 1 b$ : Increases in interpersonal distance induce feelings of spaciousness, thereby generating more self-disclosure.

In addition to the contents of self-disclosure (i.e., what do people say?), of equal importance is the affective state or the experienced ease of self-disclosure (i.e., how do participants experience the self-disclosure process?). As suggested by the foregoing, spaciousness perceptions are positively linked to the affective experience and perceived ease of self-disclosure. Hence:

H2a: Increases in room size positively influence both the affective experience and perceived ease of self-disclosure.

H1b: Increases in interpersonal distance positively influence both the affective experience and perceived ease of self-disclosure.

As discussed, ease of self-disclosure varies across topics and self-disclosure is particularly awkward during conversations on personal or intimate topics. In the healthcare or counseling context, topics of interest include physical or psychological health and health-related behaviors including substance intake and sexuality. Conceivably, for some topics (e.g., disclosure reflecting sexual behaviors) the physical presence of another person is potentially more threatening or awkward than for others (e.g., disclosure with respect to alcohol or drug intake) (cf. Howell \& Conway, 1990; Joinson, Paine, Buchanan \& Reips, 2008; Wiederman \& Sansone, 1999). Hence, for explorative and practical purposes (i.e., in healthcare and counseling settings, a large variety of topics may take center stage), a variety of topics were included in order to assess the relative importance of the spaciousness manipulations.

To test the hypotheses outlined and additional research questions, interview sessions on student lifestyle were arranged in two rooms that were identical apart from their measurements (i.e., room size), and desk selection (i.e., a small or large desk; interpersonal distance). This resulted in a 2 (room size: small versus large) by 2 (interpersonal distance: small versus large) between subjects design. 


\section{Study 4.1}

\section{Method}

\section{Participants}

A total of 86 participants (38 male, 48 female) were included in the study. Their mean age was 21.8 years $(S D=2.33)$. Participants were recruited by approaching passers-by on the campus of a Dutch university with the request to take part in a short interview concerning student lifestyle that was part of a large survey conducted by the university. All participants were students enrolled in various (under)graduate programs at the university.

\section{Procedure}

The participants were randomly assigned to one of the four conditions. Upon arrival in the building, they were asked to wait in the waiting area. After reading and signing a consent form for video recording of their interview, they were invited to enter the room. Since research suggests that self-disclosure more easily takes place towards women and between people of the same age and status (Chaikin \& Derlega, 1974; Cappella, 1981; Collins \& Miller, 1994), a female master student at the university served as the interviewer. She was not informed of the purpose of the experiment and all students that knew her were excluded from participation. The participants were interviewed according to a script, which was rehearsed by the interviewer in the weeks before the experiment. The interviewer was already present in the room and started the video recording before the participant entered the room. After entrance, the interviewer introduced herself and invited the participant to sit down in the chair after which she proceeded with the interview. Upon completion of the interview, a short questionnaire was administered (measuring spaciousness perceptions, affective experience, and perceived ease of self-disclosure) during which the participant was left alone in the room. After turning in the questionnaires, participants were thanked for their participation and dismissed.

\section{Independent variables}

The experiment took place on the campus of the university. In order to manipulate room size, two - otherwise identical - rooms of different sizes were used (see Figure 4.1 for impressions of the four experimental conditions). The small room was 16.1 square meters ( 2.80 wide $\times 5.75$ deep) and the large room was 19.78 square meters ( 4.30 wide $\times 4.60$ deep). Interpersonal distance was manipulated by varying the desk size. The interpersonal distance was $80 \mathrm{~cm}$ for the small desk size and $160 \mathrm{~cm}$ for the large desk size. A video camera was openly displayed in the corner of the room to record the interview. Lighting conditions in both rooms were measured at several different points inside the room in terms of luminance (small 
room: $M=643$ lux, range $=597-676$ lux vs. large room: $M=669$ lux, range $=590-$ 677 lux) and spectral distribution (small room: $M=2862 \mathrm{~K}$, range $=2849-2870 \mathrm{~K}$ vs. large room: $M=2856 \mathrm{~K}$, range $=2852-2869 \mathrm{~K}$ ). These differences are negligible (Kaufman, 1981). Identical decorative items were used in both rooms and their position did not change during the experiment.
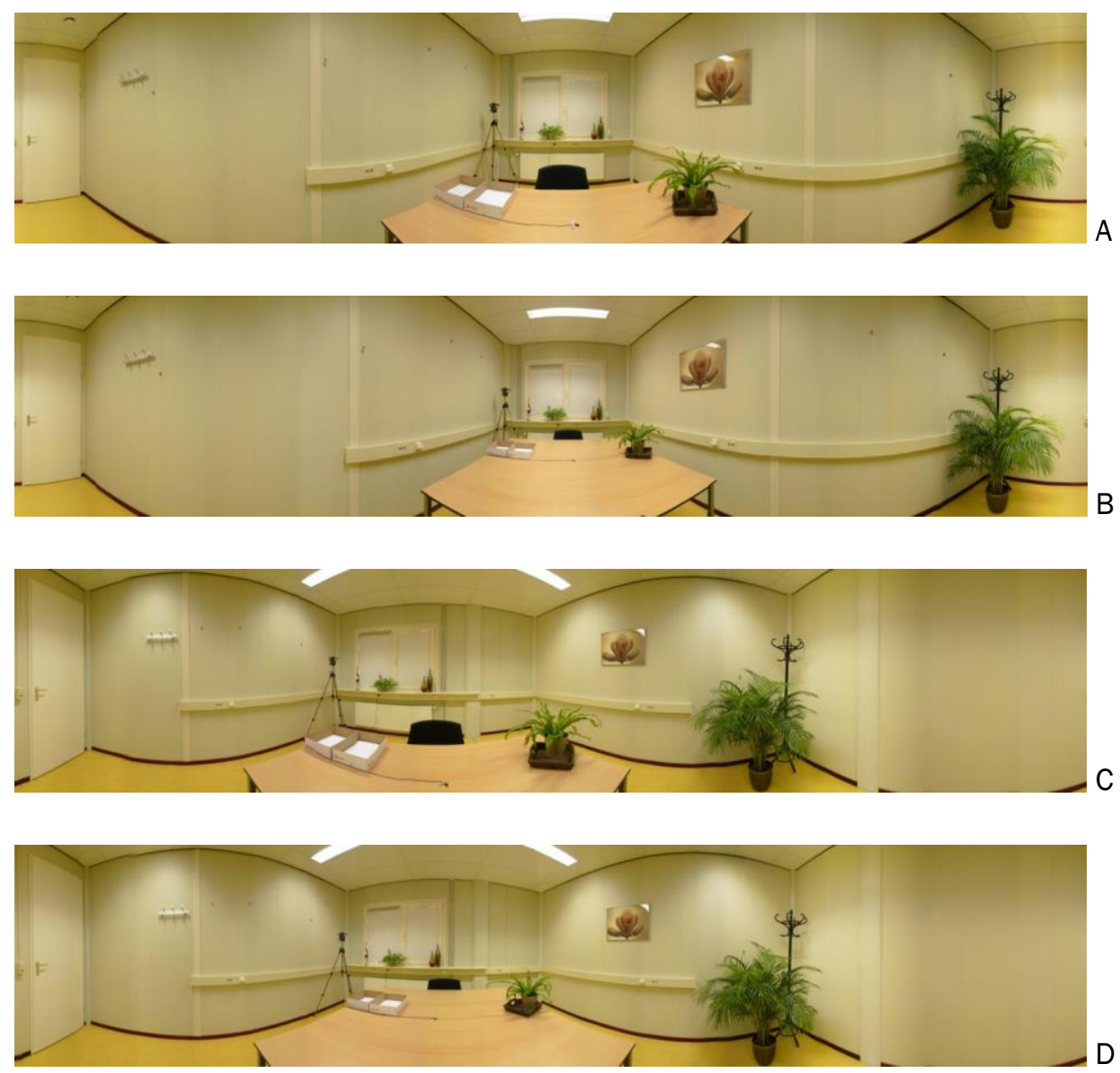

Figure 4.1. Panorama photos of the four conditions. Desk size is small for photos A and C, and large for photos $B$ and D. Room size is small for photos $A$ and $B$, and large for photos $C$ and D. 


\section{Questionnaire}

Perceived spaciousness. Perceived spaciousness was measured using the items: "I feel constricted inside this room", "I feel confined inside this room", "I have sufficient freedom of movement inside this room" and "I would easily feel suffocated inside this room" ( $\alpha=.84)$.

Perceived ease of self-disclosure. Perceived ease of self-disclosure was measured with the items: "I felt inhibited from speaking inside this room", "Inside this room I felt able to speak freely", "I felt uncomfortable in sharing personal information inside this room" and "It was hard for me to talk about myself inside this room" ( $\alpha=$ .83).

Affective experience. To measure participants' affective experience, an affectmeasure was used comprising the items: "Inside this room I feel at ease", "Inside this room I feel unhappy", "I feel uncomfortable inside this room" and "This room gives me a pleasant feeling" ( $\alpha=.84)$.

All questions were measured on five-point Likert-type rating scales.

\section{Self-disclosure measures}

Self-disclosure was measured by analyzing the answers to the questions asked during the interview. A variety of sensitive or intimate topics were selected showing an obvious match with the lifestyle-theme of the interview. Furthermore, the topic list for the interviews was based on previous research distinguishing between disclosed information in terms of sensitivity (Altman \& Taylor, 1973; Howell, \& Conway, 1990; Joinson, Paine, Buchanan \& Reips, 2008). The topics included: 'substance use' (i.e., alcohol and drug intake), 'sexuality' (i.e., attitudes about sex and mass-media), and 'emotions' (i.e., fear, insecurity and loneliness).

Substance use. Respondents were asked to describe a regular night out with their friends in terms of alcohol consumption and drug usage.

Sexuality. Respondents were asked to voice their opinion about displayed nudity and sexual behavior in the media, and to reflect on whether the media has an impact on their own sexual values and behaviors.

Emotions. Respondents were asked to describe situations in which they felt insecure, scared and lonely.

There were five measures for self-disclosure, derived from previous studies (Joinson, 2001; Omarzu, 2000). As an objective measure, word count and duration (in seconds) were measured. In addition, to gain more information about the qualitative aspects of self-disclosure, completeness (i.e., whether the participant gives a full answer to the question), self-reference (i.e., whether the participant relates the answer to him or herself), and intimacy (how intimate is the given answer) were assessed. 
To measure these qualitative aspects, recorded answers to the interview questions were scored on five-point rating scales. To determine the reliability of the ratings, a second coder re-coded a subset of the data independently using the same scales. The second coder was a behavioral scientist who was unaware of the purpose of the study. Most importantly, however, the camera was positioned so as to ensure that the raters would be blind to the conditions. The inter-coder reliability (Cohen's Kappa) varied from .70 to 1.00 indicating high inter-coder reliability.

\section{Nonverbal behavior.}

In order to obtain additional data on spaciousness-related behaviors, nonverbal behavior was coded. The measures included 'openness of posture', 'direction of bodily posture', 'leaning on the table', 'establishing eye contact', and 'bodily symptoms of distress'. Again, two independent coders viewed the recorded interview sessions and rated participants' non-verbal behaviors using five point rating scales. (Cohen's Kappa again varied from .70 to 1.00, indicating high intercoder reliability).

\section{Results}

Results were analyzed for gender and age using analysis of variance (ANOVA), but since none proved significant there will be no further discussion of these variables $(p>.10$ for all self-disclosure measures). A multivariate analysis of variance (MANOVA) was conducted, with room size and desk size as independent variables and the self-disclosure measures, perceived spaciousness, perceived ease of selfdisclosure, affective experience and the nonverbal behavior measures as dependent variables. The analysis yielded a significant multivariate effect of room size $\left(F(13,72)=2.33, p=.014\right.$, partial $\left.\eta^{2}=.29\right)$. Likewise, the multivariate effect of desk size was significant $\left(F(13,72)=3.33, p=.001\right.$, partial $\left.\eta^{2}=.39\right)$. In addition, the multivariate effect of the interaction between room size and desk size reached significance $\left(F(13,72)=2.00, p=.037\right.$, partial $\left.\eta^{2}=.26\right)$. Having established these effects, next the ANOVAs (comprising the same independent and dependent variables) are presented.

\section{Perceived spaciousness}

Room size had an effect on perceived spaciousness $(F(1,84)=6,60 ; p=.012$, partial $\left.\eta^{2}=.07\right)$. As expected, perceived spaciousness scores for the large room $(M$ $=5.81, S D=.17)$ were higher than for the small room $(M=5.19, S D=.17)$, indicating that participants experienced more spaciousness in the large room than in the small room. No main effect was found for desk size ( $F<1, n s)$.

An interaction was obtained between desk size and room size $(F(1,82)=9.03 ; p=$ .004 , partial $\eta^{2}=.10$ ) (see Figure 4.2). Further analysis of the simple main effects 
showed that for the large desk size, there was a significant difference in perceived spaciousness for the different room sizes (small room size $M=4.82, S D=.23$ vs. large room size $M=6.15, S D=.24 ; F(1,82)=16.15, p<.000$, partial $\left.\eta^{2}=.17\right)$, indicating that participants experienced the condition with the larger desk size as more spacious in the large room, as compared to the small room. In the small desk condition, this difference did not reach significance (small room $M=5.54, S D=.22$ vs. large room $M=5.48, S D=.23 ; F<1, n s$ ).

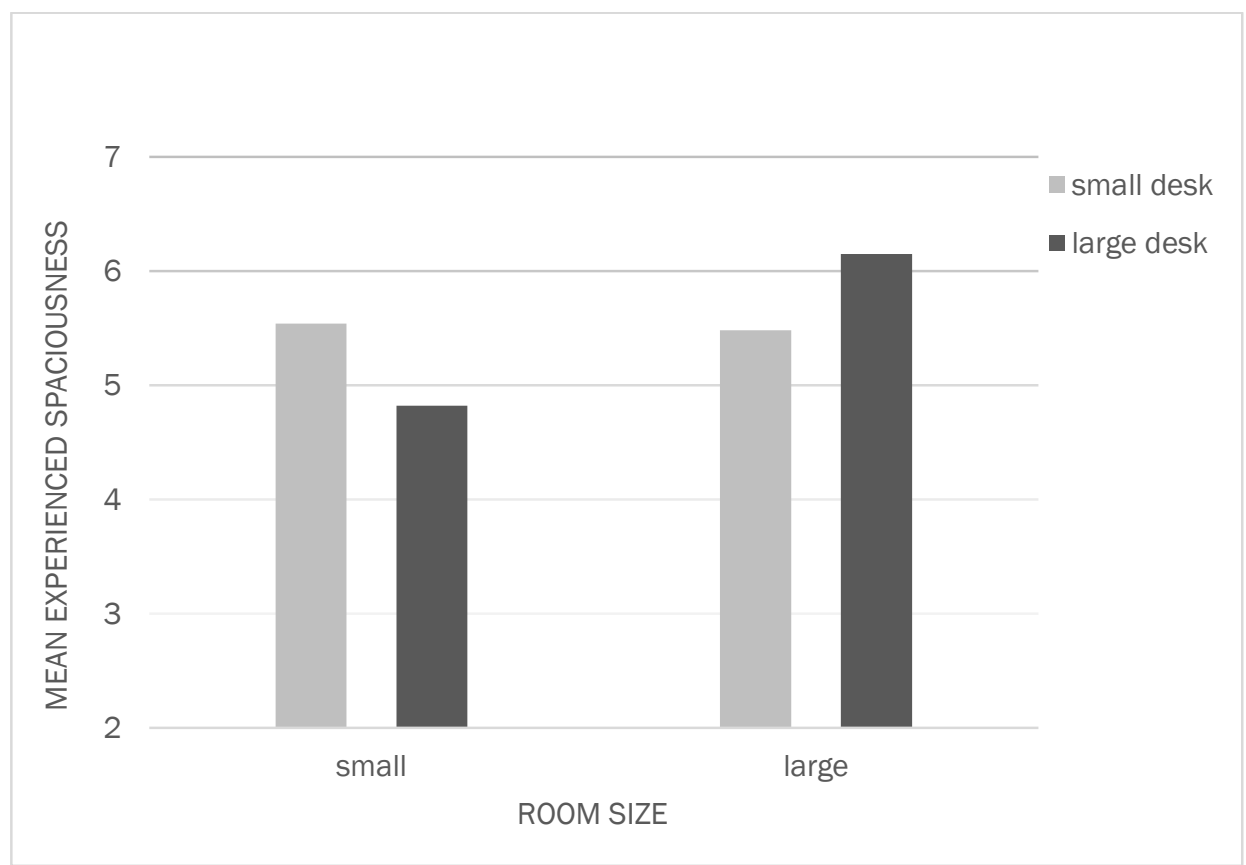

Figure 4.2. Interaction between room size and desk size for perceived spaciousness.

\section{Affective experience}

Perceived ease of self-disclosure. Room size had a significant effect on perceived ease of self-disclosure $\left(F(1,84)=4.23, p=.042\right.$, partial $\left.\eta^{2}=.05\right)$. The large room yielded higher scores for ease of self-disclosure $(M=5.01, S D=.14)$ in comparison to the small room $(M=5.50, S D=.15)$. No main effect was found for desk size $(F<$ $1, n s)$. The interaction between desk size and room size was also significant ( $F(1$, $82)=4.36 ; p=.040$, partial $\eta^{2}=.05$ ) (see Figure 4.3). Further analysis of the simple main effects showed that the positive effect of room size on self-disclosure was significant only in the large desk condition (small room $M=4.96, S D=.20$ vs. large room $M=5.79, S D=.21 ; F(1,82)=8.4, p=.005$, partial $\left.\eta^{2}=.09\right)$. In the small desk condition, the effect of room size was not significant (small room $M=$ $5.21, S D=.20$ vs. large room $M=5.20, S D=.20, F<1, n s)$. 


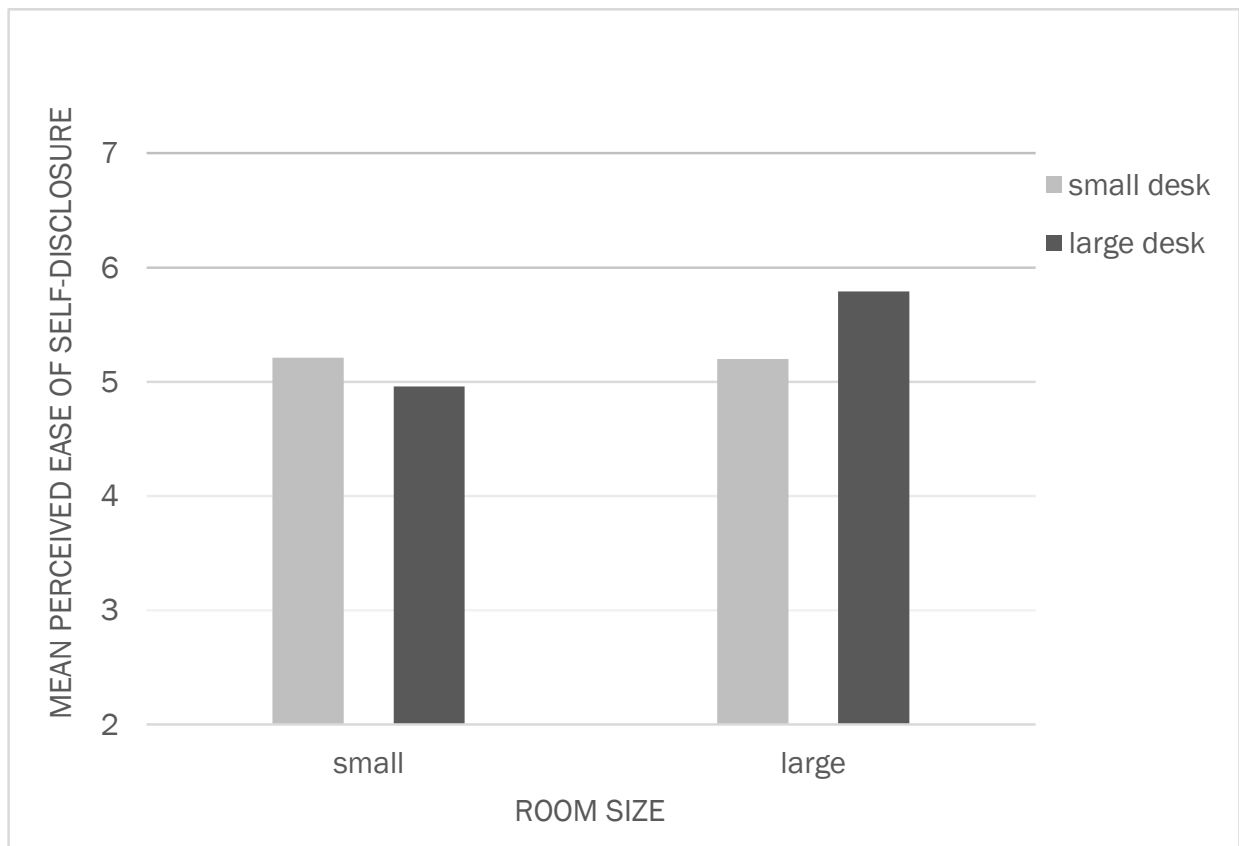

Figure 4.3. Interaction between room size and desk size for the perceived ease of selfdisclosure.

Affective experience. Room size had significant effect on affective experience ( $F$ $(1,84)=4.78, p=.032$, partial $\left.\eta^{2}=.05\right)$. The large room triggered a more positive affect $(M=5.21, S D=.16)$ than the small room $(M=4.73, S D=.15)$. For desk size and the room size $x$ desk size interaction no significant effects were obtained $(F<1$, $n s)$.

Mediation analyses, following the procedure outlined by Baron and Kenny (1986), were performed in order to examine whether the effects of room size and the room size $x$ desk size interaction on perceived ease of self-disclosure and affective experience are mediated by perceived spaciousness (see Figure 4.4). Starting with ease of self-disclosure, the effects of room size on perceived ease of self-disclosure $(\beta=.21, p=.047$ ) and on perceived spaciousness (mediator) were significant ( $\beta=$ $.27, p=.012$ ), as was the effect of perceived spaciousness on perceived ease of self-disclosure $(\beta=.59, p<.000)$. When both room size and perceived spaciousness were included in the model, the effect of room size on perceived ease of self-disclosure became non-significant $(\beta=.06, p=.541)$, whereas the effect of perceived spaciousness on perceived ease of self-disclosure remained significant ( $\beta$ $=.57, p<.000$ ). Results of a Sobel test show that the indirect effect is significant (Sobel $z=2.40, p=.008$ ). 


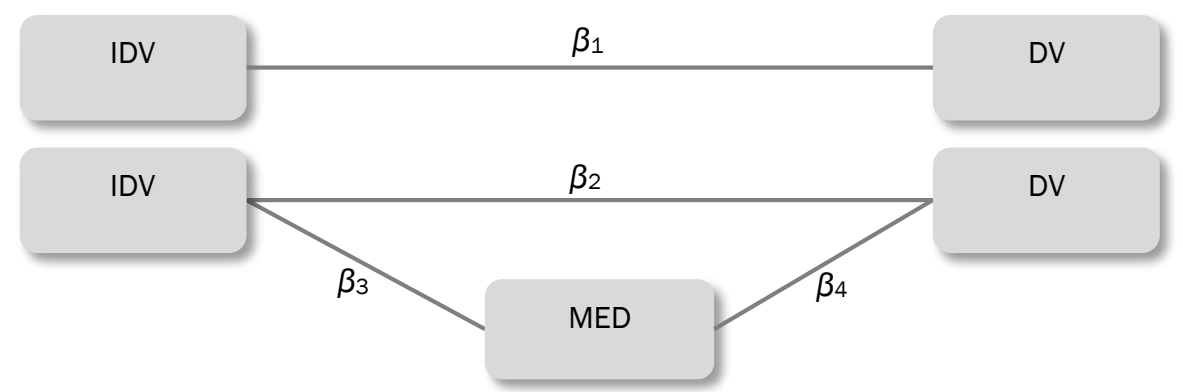

Figure 4.4. Mediation model, with room size or the room size $\mathrm{x}$ desk size interaction as the independent variable (IDV), perceived spaciousness as the mediator (MED), and selfdisclosure, perceived ease of self-disclosure and affect as dependent variable (VD).

In order to test whether the main effect of room size on affective experience is mediated by spaciousness, similar analyses were conducted (see Table 4.1). Taken together, these analyses confirm that people feel more at ease self-disclosing personal information and experience more positive affect in the large setting because it inspires spaciousness perceptions.

\section{Self-disclosure}

No overall effects on the self-disclosure measures surfaced across the different topics, suggesting that the effects of the space manipulations varied depending on the topic of conversation. Hence, separate analyses were conducted for each of the three topics: substance use, sexuality and emotions respectively.

Substance use. Room size had an effect on word count for the questions related to substance use $\left(F(1,83)=5.30, p=.024\right.$, partial $\left.\eta^{2}=.06\right)$. The amount of words used to answer the substance use questions was lower in the small room as compared to the large room. No effects surfaced on the other self-disclosure measures (see Table 4.2).

Sexuality. Desk size had an effect on word count for the questions related to sexuality $\left(F(1,83)=4.46, p=.038\right.$, partial $\left.\eta^{2}=.05\right)$. The number of words used was higher in the large desk condition compared to the small desk condition. Also, desk size had a marginal effect on the duration of the answer $(F(1,83)=2.91, p=$ .092, partial $\left.\eta^{2}=.03\right)$. Participants' answers took more time in the large desk setting compared to the small desk setting. Desk size had an effect on selfreference for the sexuality questions $\left(F(1,83)=5.19, p=.025\right.$, partial $\left.\eta^{2}=.06\right)$. Participants' answers were more self-related, and less other-related, at the large desk compared to the small desk. 


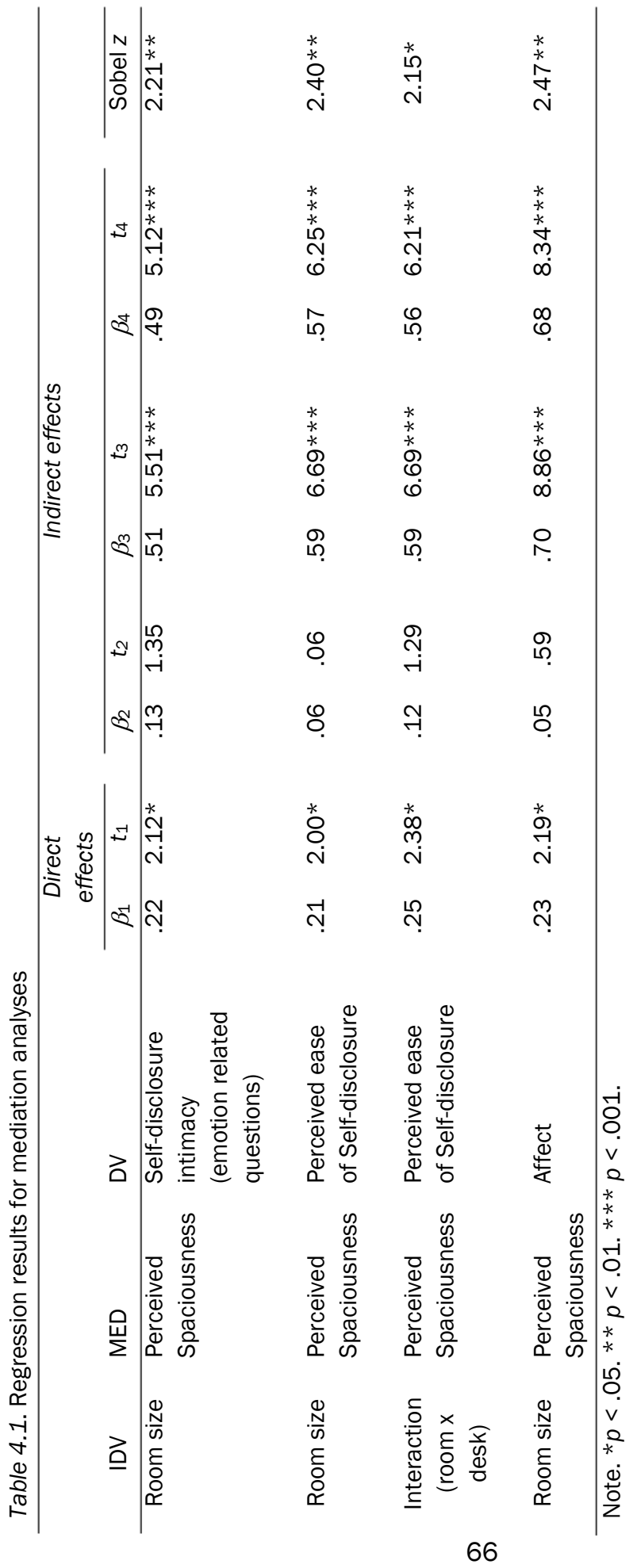


Emotions. Room size had a marginal effect on intimacy for the questions related to emotions $\left(F(1,84)=4.75, p=.020\right.$, partial $\left.\eta^{2}=.05\right)$. Answers were more intimate in the large room, as compared to the small room.

Mediation. In order to test whether the effects obtained for self-disclosure were mediated by spaciousness, mediation analyses were conducted (see Table 4.1). No overall mediating effects of perceived spaciousness were found. However, for the emotion questions, a significant mediation was found for the intimacy of the given answer. The effects of room size on intimacy $(\beta=.22, p=.035)$ and on perceived spaciousness ( $\beta=.20, p=.048$ ) were significant, as was the effect of perceived spaciousness on intimacy $(\beta=.51, p=<.000)$. When both room size and perceived spaciousness were included in the model, the effect of room size on intimacy became non-significant $(\beta=.13, p=.180$ ), whereas the effect of perceived spaciousness on intimacy remained significant $(\beta=.49, p<.000)$. Results of a Sobel test show that the indirect effect is significant (Sobel $z=2.21, p=.018$ ). In other words, respondents disclosed more intimate information in the large room because they perceive the room as more spacious.

\section{Nonverbal behavior}

Room size had an effect on direction of bodily posture $(F(1,84)=5.27, p=.024$, partial $\left.\eta^{2}=.06\right)$. Participants leaned more forwards in the large room $(M=1.93, S D$ $=.03)$ than in the small room $(M=2.05, S D=.03)$. Also, room size had an effect on openness of posture $\left(F(1,84)=5.56, p=.021\right.$, partial $\left.\eta^{2}=.06\right)$. Participants displayed a more open posture in the large room $(M=3.84, S D=.09)$ than in the small room $(M=3.51, S D=.09)$.

Desk size affected the extent to which participants leaned on the table $(F(1,84)=$ 40.02, $p<.000$, partial $\eta^{2}=.32$ ). Participants leaned more on the table when seated at a large desk $(M=2.61, S D=.12)$ compared to a small desk $(M=1.53$, $S D=.12$ ). 


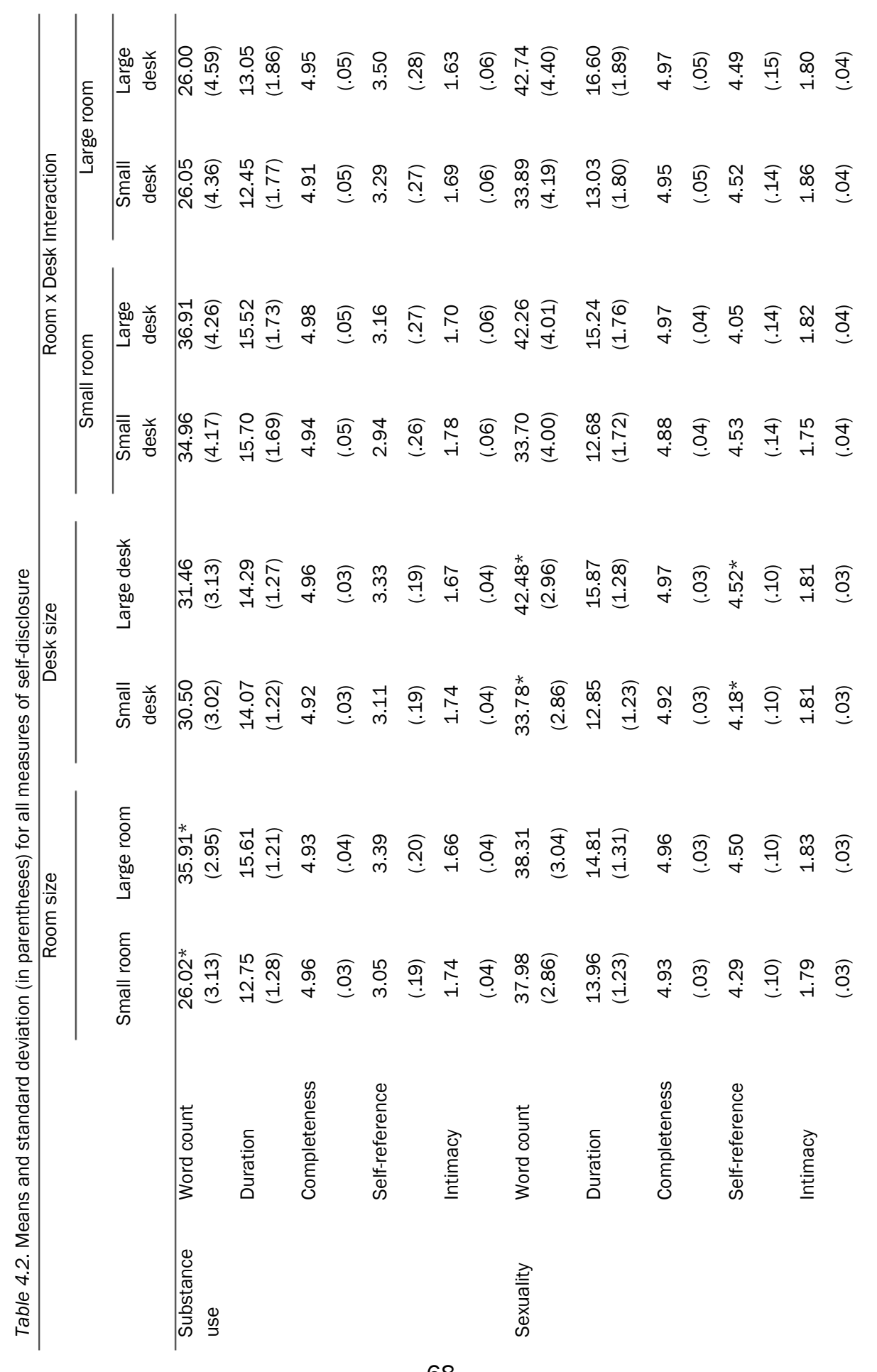




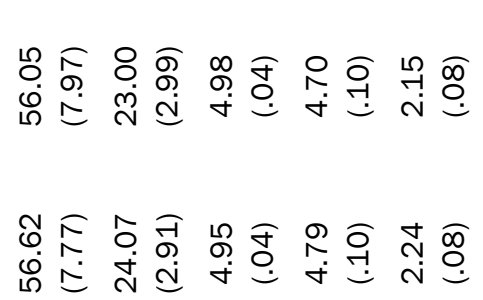

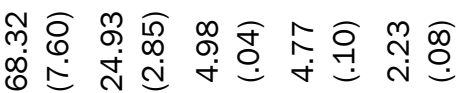

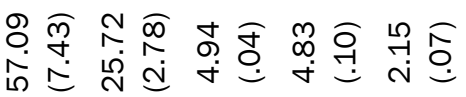

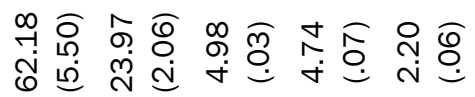

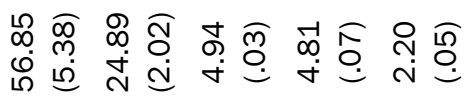

유 감 ชิ

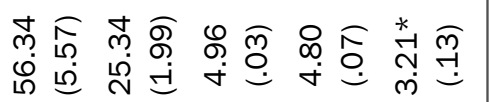

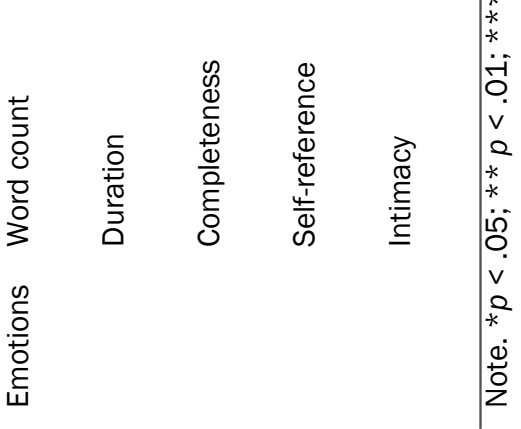


An interaction between room size and desk size was obtained for establishing eye contact $\left(F(1,81)=6.33 p=.01\right.$, partial $\eta^{2}=.07$ ) (see Figure 4.5). Further analysis of the simple main effects showed that the positive effect of room size on eye contact was only significant in the large room condition (small desk $M=3.57, S D=$ .10 vs. large desk $M=3.87, S D=.10 ; F(1,81)=4.09, p=.04$, partial $\left.\eta^{2}=.05\right)$. In the small room condition, no significant difference was found for the effect of desk size (small desk $M=3.90, S D=.10$ vs. large desk $M=3.69, S D=.10 ; F(1,81)=$ $2.32, p=.132$ ).

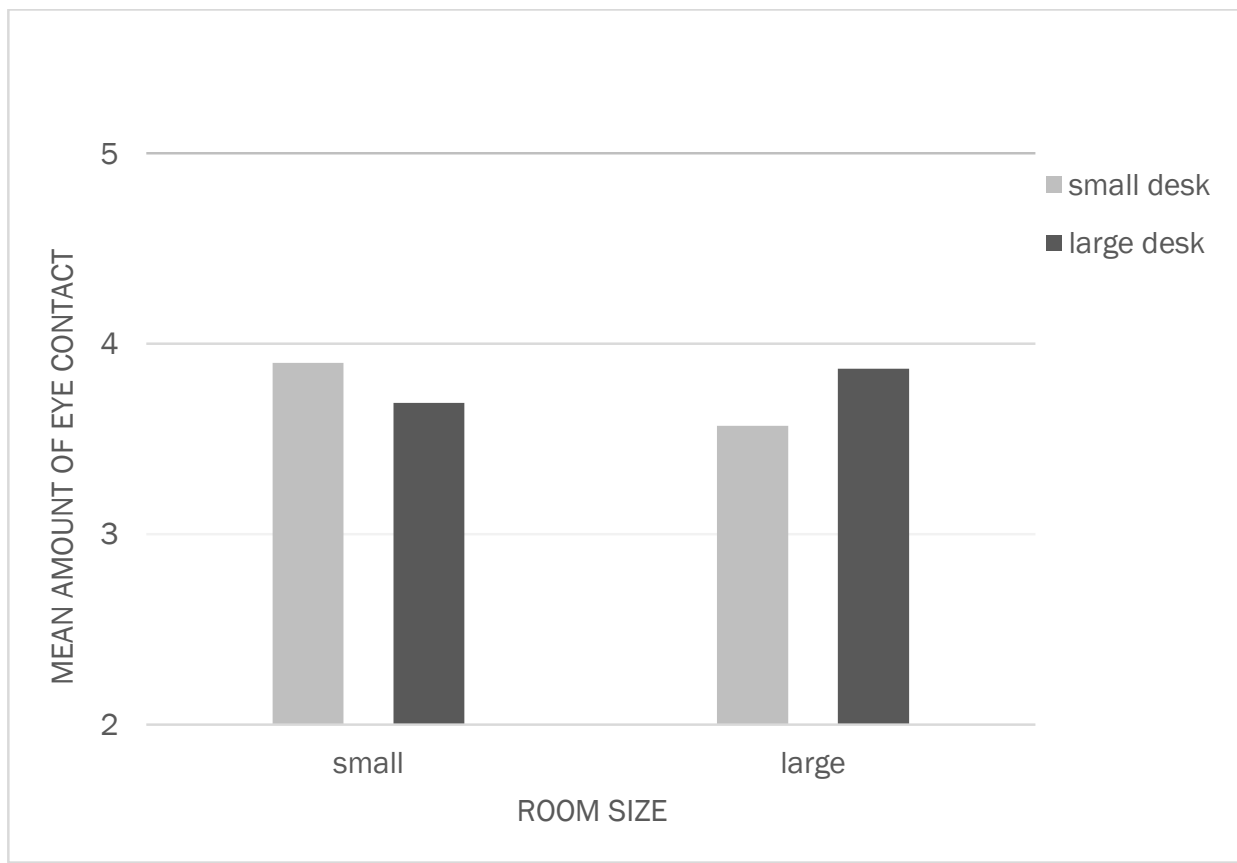

Figure 4.5. Interaction between room size and desk size for the amount of eye contact.

\section{Discussion}

The results presented confirm the importance of spaciousness manipulations with respect to the affective experience and the ease of self-disclosure. Desk size and room size in particular were found to impact spaciousness perceptions. Contrary to expectations, however, the effect of spaciousness did not transpire in the small desk setting. A possible explanation is that the small interpersonal distance directs the focus towards the interviewer and away from the architectural design of the room. In line with this notion, Albert and Dabbs (1970) showed that at a small interpersonal distance, people generally pay more attention to the physical appearance of the speaker than to the spoken message itself. Thus because the 
physical closeness of another person can pose a threat to peoples' personal space (Sommer, 2007), this may create 'tunnel vision' that makes factors such as the conversation and the environment much less relevant than the physical appearance and behaviors of the conversational partner (cf. Easterbrook, 1959; Friedman \& Förster, 2010).

In sum, the room size and interpersonal distance manipulations had an impact on both the affective experience and the ease with which participants self-disclose. Subsequent mediation analyses confirmed that spaciousness perceptions are at the basis of these effects. However, for the actual self-disclosure measures, the results paint a much less coherent picture; the effects varied considerably across the topics and relatively few effects were obtained. It thus appears that reported ease of self-disclosure and actual disclosing behavior might paint a different picture, or that enhanced ease of self-disclosure and accompanying positive affect do not necessarily translate into more objective measures such as duration and word count. Nonetheless, several findings merit further attention and suggest interesting avenues for follow-up research.

First of all, the results clearly stress the importance of taking into account the topic of conversation when studying self-disclosure as a function of the environmental setting. Most notably, in response to questions about substance use and emotions, room size was of primary importance, but when answering questions related to sexuality, desk size (i.e., interpersonal distance) appeared to be the critical factor. Arguably, when discussing activities involving a strong physical component, the physical closeness of another human being becomes salient and poses a greater threat, redirecting the focus towards this other person and away from environmental aspects such as room size (Easterbrook, 1959; Friedman \& Förster, 2010; Sommer, 2007). In addition, research indicates that discussing sexuality remains a personal, often taboo, topic that induces anxiety and embarrassment, especially in counseling settings (Joinson, Paine, Buchanan \& Reips, 2008; Wiederman \& Sansone, 1999).

Furthermore, the results suggest that depending on topic, different aspects of selfdisclosure take center stage. For the substance use questions, for instance, word count was affected by room size with more words being spoken in the large setting. A possible explanation holds that when discussing alcohol and drug intake, bragging may be involved (Jung, 2001, Martin \& Leary, 2001) and that respondents talk more extensively about their intake behaviors in a less restrictive (i.e., more spacious) environment. For sexuality, on the other hand, effects of desk size on word count, duration of the answer and self-reference surfaced. As mentioned previously, discussing sexual issues with an unknown person is very sensitive and likely influenced by the spaciousness of the situation (Joinson, Paine, Buchanan \& 
Reips, 2008; Wiederman \& Sansone, 1999). In addition to giving a more extensive answer, using more self-references can indicate an increased ease to discuss this taboo topic, while using more other-references may be considered an indication of avoidance. Finally, for the emotion questions, the intimacy of participants' responses was affected (with less intimate answers characterizing the small environment). This is likely due to the fact that both a small environment and the sharing of negative emotional experiences invoke feelings of intimacy (Howell \& Conway, 1990). This caused respondents in the small environment to limit the amount of intimate information and answer in more general terms to prevent the situation from getting too intimate or, as suggested by Levav and Zhu (2009) to regain their freedom.

Although such explanations are admittedly speculative, these results do show that measuring self-disclosing behavior with a single measure or failing to consider the conversational context may lead one to miss out on potentially interesting effects. Of course, the set of measures used in this research is far from exhaustive, and in other settings different or additional measures may turn out to be of relevance. For instance, 'level of detail' may be a relevant measure in conversations on therapy loyalty or occurrences of physical complaints, but also in eyewitness reports (a context in which self-disclosure is also of primary importance).

The effects observed for nonverbal behaviors further suggest that manipulations of interpersonal distance may evoke readjustments (e.g., leaning forward) to restore appropriate spacing between conversation partners. Interestingly, room size also affected nonverbal behaviors (i.e., leaning forward and alterations of posture). The reported results suggest that a larger room (i.e., the presence of more overall space) is an invitation to use or claim more space by leaning further forward and by adopting a more open bodily posture. In addition, participants established more eye contact in the large room, likewise suggesting an increased willingness to interact.

In the current study, a female master student conducted the interviews; a deliberate choice as research shows that disclosure more easily takes place towards women and between people of similar age or status (Chaikin \& Derlega, 1974; Cappella, 1981; Collins \& Miller, 1994). In line with earlier research (Dindia \& Allen, 1992), in this study no effects were found for gender and for age. However, this should not obscure that interviewer characteristics may affect self-disclosure tendencies. For instance, in health settings differences in expertise and authority between patient and physician may withhold patients to freely disclose personal information, as suggested by recent studies showing that both environmental preferences and displayed behaviors are influenced by the presence of a threatening person (Wyer \& Calvini, 2011). In addition, participants in the study described were all students and thus relatively young; participants of older age are 
perhaps more sensitive to characteristics such as gender of their conversation partner. Also, the used rooms were existing rooms on the campus of the university. Besides the difference in square footage, however, the settings used also varied to some extent in terms of proportion, with the small room being more rectangularshaped than the large room. We did not examine whether the proportions of the room influenced the effects obtained. However, considering the findings of Daves and Swaffer (1971), future research should examine the effects of room proportion on affective and behavioral outcomes. In addition, although the results presented in this paper indicate that relative differences in room size and desk size affect both affective and behavioral outcomes, they do not warrant any conclusions regarding absolute room size.

As for practical implications, the differences in effects per topic call for adopting a flexible environment (i.e., extendible desks) that can be easily altered to fit the needs of a large variation of conversations. To influence room size, room dividers may be used when resorting to another (smaller or larger) room is not an option. Furthermore, room layout and positioning of other furniture pieces can influence the amount of space available and in turn possibly influence self-disclosing behavior. Of interest in this context are the results of Stamps and Krishnan (2006) which show that spatial density (i.e., the number of objects present in a limited space) can also influence spaciousness perceptions and thus perhaps also self-disclosure, with the more furnished room invoking more spaciousness than the empty room. In addition, research indicates that dependent on the type of objects (e.g., professional versus decorative objects), environmental settings and their inhabitants may come across as more or less homelike or professional (Verhoeven, van Rompay \& Pruyn, 2007), a factor that may likewise influence self-disclosure (Gifford, 1988; Miwa \& Hanyu, 2006).

Furthermore, the results reported per topic indicate that the function of the room should be taken into account when designing and furnishing a room. For although room size was most important with respect to substance use and emotions, sexual self-disclosure was clearly affected by interpersonal distance, a clear indication that a relational or sex therapist might best keep in mind the importance of interpersonal distance when deciding on room layout and furniture selection. In light of this notion, of interest is the finding that alterations of posture (e.g., leaning on the table) may be used to adjust interpersonal distance. Clearly this is something that physicians and counselors should be aware of, especially when discussing more intimate topics for which a large interpersonal distance is desirable.

Finally, in addition to objectively manipulating available space, room atmospherics (which allow for easy and flexible adjustments) can also be used to create illusions of a larger or smaller room and, hence, may in turn influence self-disclosure. In line 
with this suggestion, both Gifford (1988) and Miwa and Hanyu (2006) showed that lighting conditions can affect disclosure tendencies. In light of the findings presented in this paper, of particular interest are effects of atmospheric variables such as lighting and color that have been shown to affect spaciousness perceptions (Stamps, 2011). Arguably, bright colors or lighting conditions may foster the impression of a spacious environment, thereby promoting self-disclosure. Awaiting future research addressing these and other variables, the results of the present study confirm the importance of a neglected environmental variable with respect to a key facet of interpersonal behavior. 
The previous chapters, Chapter 3 and 4, demonstrated beneficial effects of a more spacious environment on self-disclosure. Moreover, they show that variables on both an architectural level and an interior design level can be used to alter the degree of perceived spaciousness of an environment. Chapter 5 investigates whether, besides such tactile elements, room atmospherics can also be used to impact self-disclosure intentions via perceived spaciousness. The experiment reported investigates whether room brightness can be used to create the illusion of a more spacious (or less spacious) environment without manipulating the actual measurements of the room. The results of this study confirm that room brightness impacts affective experiences and self-disclosure intentions via experienced spaciousness. In addition, it was found that this relationship is influenced by psychological circumstances, with a more pronounced need for space when in an anxious state of mind. Finally, the results show that perception of the depicted physician is also influenced by the brightness manipulation. 


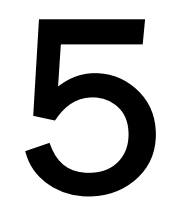

\section{When the world is closing in: Effects of}

\section{perceived room brightness and communicated threat during patient-physician interaction ${ }^{4}$}

4 Okken, V. S., van Rompay, T. J. L., \& Pruyn, A. Th. H. (in press). When the world is closing in. Effects of perceived room brightness and communicated threat during patient-physician interaction. Health Environments Research and Design Journal. 
"I think night time is dark so you can imagine your fears with less distraction."

(Calvin in The Indispensable Calvin and Hobbes, Bill Watterson, 1992)

\section{Introduction}

Imagine yourself entering an unfamiliar room for the first time. Whether it is a colleague's apartment, a private physician's practice or a counseling environment, you can often instantly tell whether the room provides you with enough 'breathing space' or 'freedom of movement'. Dependent on such impressions, you are likely to feel 'confined', 'secure' or 'lost', and you may feel the urge to stay, explore the space, interact with others, or, alternatively, leave as soon as possible. Sometimes, however, the room that was spacious on one occasion may feel less 'roomy' on another, or put differently, the walls that once were at a safe distance now suddenly are 'closing in'. What this example suggests is that perceptions of spaciousness are not only the resultant of architecture and interior design, but vary with the visitor's state of mind as well.

In line with this example, research shows that spatial aspects of environmental settings play an important part in influencing affective experiences and behaviors. Sundstrom (1975) showed that limited physical space induces crowding perceptions, which in turn may decrease communicative behavior. Okken, Van Rompay and Pruyn (2012) examined the effects of limited space during patientphysician interaction by altering room size and interpersonal distance. Their results showed that participants who felt physically restricted expressed a lowered willingness to self-disclose. From a practical point of view, however, manipulations related to architectural or interior design elements are often troublesome; room size is usually fixed, and factors such as room layout and furniture selection are not always under the control of healthcare providers either. Of particular interest to current undertaking, however, is the finding that atmospheric variables such as color and lighting may also foster perceptions of spaciousness (Stamps, 2011) and related feelings (Akalin-Baskaya \& Yildirim, 2007) and behaviors (Baron, Rea \& Daniels, 1992). Examining the effects of such environmental influences in a counseling or healthcare context can possibly provide more easily adaptable and flexible tools to create the 'right' atmosphere. In turn, this may improve the diagnostic process by increasing patients' active participation during conversations with their physician. Hence, in current research we addressed the relationship between perceived room brightness (fostering perceptions of a more or less spacious environment) on the one hand and experienced affect and behavioral intentions on the other. More specifically, to extend knowledge about the influence of the physical environment on patient-physician communication, this study 
investigated effects of perceived brightness on the disclosure of personal information during a simulated patient-physician conversation. In addition, we studied whether effects of perceived brightness are qualified by the patient's state of mind, and more in particular, with the extent to which a patient feels threatened or relieved.

\section{Environmental factors and spaciousness perceptions}

Research suggests that perceived spaciousness is important to inhabitants across environmental settings because it inspires feelings of freedom. For instance, Meyers-Levy and Zhu (2007) showed that a high, as opposed to a low, ceiling activated feelings of freedom and spaciousness and more creative strategies for problem solving tasks. In a similar fashion, Levav and Zhu (2009) examined the effects of experienced spaciousness in store environments and showed that narrow aisles activate feelings of confinement, which consumers counteracted by making more varied product choices. These findings suggest (in line with embodiment research; IJzerman \& Semin, 2010; Williams \& Bargh, 2008) that restraining physical space invokes feelings of limited psychological space. In turn, these negative feelings of restraint may cause reactance, emerging as a refusal to cooperate or comply with behavioral norms or to display expected behavior.

In addition to variations related to tangible or physical parameters, these perceptions may also vary with room atmospherics such as color (Acking \& Küller, 1972; Kwallek, 1996; Oberfeld, Hecht \& Gamer, 2010; Yildirim, Akalin-Baskaya \& Hidayetoglu, 2007) and lighting (Durak, Olgonturk, Yener, Guvenc \& Gurcinar, 2007; Flynn, Spencer, Martyniuk \& Hendrick, 1973; Hidayetoglu, Yildirim \& Akalin, 2012; Manav 2007). For instance, Acking and Küller (1972), who repainted dayrooms in a hospital in different colors, found that a white room was judged as more open, compared to a light-green and a dark-green room. In line with these findings, Kwallek (1996) revealed that a white wall color received the highest spaciousness scores, in comparison to darker colors such as green and red. Oberfeld, Hecht and Gamer (2010) showed that not only a brighter wall color, but also brighter ceiling increases perceived spaciousness. Also, the relationship between lighting and perceived spaciousness has received considerable attention. Flynn, Spencer, Martyniuk and Hendrick (1973) showed that spaciousness judgments differed significantly for rooms with different lighting conditions. More specifically, lighting all four walls (compared with overhead lighting merely lighting the center of the room) induced greater feelings of spaciousness. Durak et al. (2007) varied room brightness and found that the brighter condition was judged as more spacious. Finally, Manav (2007) investigated lighting conditions in an office setting and 
showed that brighter lighting conditions received higher scores for comfort and spaciousness compared to more dimmed conditions.

These findings suggest that spaciousness perceptions vary depending on lighting conditions and color selection. Nevertheless, understanding of how and why atmospherics influence brightness perceptions is still limited. In addition, atmospheric factors such as color and lighting are complex stimuli, making it hard to pinpoint what exactly accounted for the effects observed (cf. Valdez \& Mehrabian, 1994). Arguably, perceived room brightness is a key variable in explaining effects of room atmospherics, with brighter surroundings conveying the impression of a more spacious environment. The rationale behind this line of reasoning holds that a brighter, as opposed to a darker, environment provides higher levels of perceptual clarity and that increases of perceptual clarity make an environment come across as more spacious (cf. Flynn, Spencer, Martyniuk \& Hendrick, 1973). This is in line with findings of Hidayetoglu, Yildirim and Akalin (2012), showing that a brightly lit environment positively affects perceptual clarity of the environment and facilitates navigation and way finding therein. Also, when looking at our own experience we find that we can better survey our environment during daytime, and that we can see more of our surroundings in daylight compared to nighttime.

\section{Perceived brightness and self-disclosure}

Previous research indicates that variations in lighting conditions affect selfdisclosure (e.g., Gifford, 1988; Miwa \& Hanyu, 2006). For instance, Gifford (1988) showed that bright lighting stimulated both general and intimate communication of participants. In this paper it is argued that effects of brightness on self-disclosure are mediated by spaciousness perceptions with brighter surroundings creating the illusion of a more spacious environment. As physical freedom triggers positively charged perceptions of psychological freedom, people are more likely to cooperate with requests (cf. Meyers-Levy \& Zhu, 2007; Levav \& Zhu, 2009), and hence may self-disclose more easily. Darker lighting conditions, on the other hand, may cause (negatively charged) feelings of insufficient space and reduced freedom, in turn increasing reactance and hence lowering the willingness to self-disclose. Hence the first hypothesis states:

H1: An increase in perceived brightness induces perceptions of spaciousness $(\mathrm{H} 1 \mathrm{a})$, thereby generating more positive affect $(H 1 b)$ and enhancing self-disclosure intentions $(H 1 c)$. 
However, as suggested, in some cases darker surroundings may promote selfdisclosure, arguably because they create a more intimate environment (Miwa \& Hanyu, 2006). In addition, research suggests that feelings of reduced spaciousness may be preferable in terms of affect and behavior when the conversational context is stress-free. For instance, Greene (1977) showed that when receiving positive feedback, a smaller distance between conversation partners invoked more positive affect. Furthermore, Schiffenbauer and Schiavo (1976) showed that a smaller interpersonal distance promotes likeability during a positive conversation, whereas during a negative conversation, a larger distance invokes a more positive response. Finally, a study by Dosey and Meisels (1969) showed that participants maintain a larger distance between themselves and an interviewer in high-stress, as opposed to low-stress, situations. In line with these findings, Okken, van Rompay and Pruyn (2012) showed that during a positive conversation the need for space is less pronounced than during a negative conversation.

At this point, one could wonder whether a non-threatening situation merely reduces spaciousness needs or whether a patient's state of mind influences spaciousness perceptions in the first place. Although this question has not yet been tested explicitly in the context of healthcare services, the idea that the state of mind influences environmental perception is commonly accepted in other, related areas of research. For instance, results of Hui and Bateson (1991) indicate that experiencing more control makes a service environment seem less crowded (cf. Baum, Fisher \& Solomon, 1981). Inspired by research indicating that one's state of mind steers environmental perception, here it is argued that:

H2: Effects of perceived brightness on perceived spaciousness $(H 2 a)$, affective experience $(\mathrm{H} 2 b)$ and intended self-disclosure $(\mathrm{H} 2 \mathrm{c})$ are more pronounced during a threatening conversation, as opposed to a non-threatening conversation.

Finally, research indicates that effects of environmental variables can translate to respondents' judgments of other persons present in the room. For instance, a classic study of Maslow and Mintz (1956) showed that people consider faces more attractive when presented in an aesthetically pleasing, as opposed to an ugly, room. Similar results were found by Teven and Comadena (1996), who studied the effect of the aesthetic quality of a teacher's office on, among others, evaluations of teacher credibility and communication style. Results showed that room aesthetics positively influenced credibility ratings and translated to more positive judgments of communication style. These findings suggest that positive affect inspired by room atmospherics may positively influence person perception. Hence, for explorative purposes we will test the prediction that: 
H3: The positive effects induced by room brightness translate to higher ratings of physician likeability.

To test the hypotheses outlined above, perceived brightness was manipulated using pictures of a consultation room, and the level of communicated threat by constructing two variants of a scenario (i.e., a low threat and a high treat scenario), resulting in a 2 (perceived brightness: bright vs. dark) x 2 (communicated threat: low vs. high) between-subjects design.

\section{Study 5.1}

\section{Method}

Participants and procedure

A total of 90 participants (33 male, 57 female; mean age 20.94 years, $S D=2.25$ ) participated. They were recruited by approaching passers-by on the campus of a Dutch university with the request to participate in a study on their impression of healthcare environments. All participants were students enrolled in various (under)graduate programs at the university. In the introduction, participants were informed that the purpose of the study was to extend knowledge about patients' judgments of consultation rooms of general practitioners and specialists in hospitals. Next, they were presented with one of two possible scenarios varying in communicated threat. The low threat condition presented a conversation with a doctor following a 'nothing to worry' checkup, whereas the high threat condition presented a conversation following a more troublesome checkup. A manipulation check confirmed the intended difference between the scenarios $(F(1,89)=15.31$; $p<.001$ ) displayed below (Note: Manipulations are displayed in bold typeface; wording used in the high threat condition are between brackets).

About eight weeks ago you visited your general practitioner because you experienced skin irritations on your abdomen and back. You were referred to a dermatologist at the local hospital. In the following period, several medical tests took place and the dermatologist provided you with a zinc ointment to rub on the irritated parts of your body. The treatment appears (not) to work because you experience less itching (more itching) and the irritation has almost disappeared (seems to increase). Last week you had a telephone consultation with your dermatologist. He informed you that the test results of the latest test are in and that it appears to be nothing serious (and that the results are inconclusive). Today you have an appointment with the dermatologist for a discussion of the test results, your experiences and an additional check-up. You feel relieved (worried) because 
the itching and irritation pose no serious threat (the source of your complaints is unclear), and have almost disappeared (that it is hard to assess the seriousness of the health threat involved).

Next, the participants were presented with a picture of one of two possible consultation rooms and asked to imagine themselves in the situation depicted (see Figure 5.1). In order to manipulate perceived brightness, one template of a consultation room was used, of which the brightness of the back wall was modified. A pilot study was conducted in which 10 participants were shown a series of pictures with different brightness values and asked to indicate what they considered a realistic setting for a patient-physician conversation. Based on these results, one picture was selected for the bright condition and one picture for the dark condition. The difference in brightness value was sixty percent (RGB values dark vs. bright: $137,133,129$ vs. $189,185,180)$. In order to control for a possible confound of aesthetic impression, participants of the pilot study were also asked to judge the aesthetics of the pictures. Results showed that the selected pictures do not differ in this regard $(F<1, n s)$. Next, the questionnaire was presented, comprising the dependent variables perceived spaciousness, affective experience and intended self-disclosure. Upon completion of the questionnaire, participants were thanked for their cooperation and dismissed.
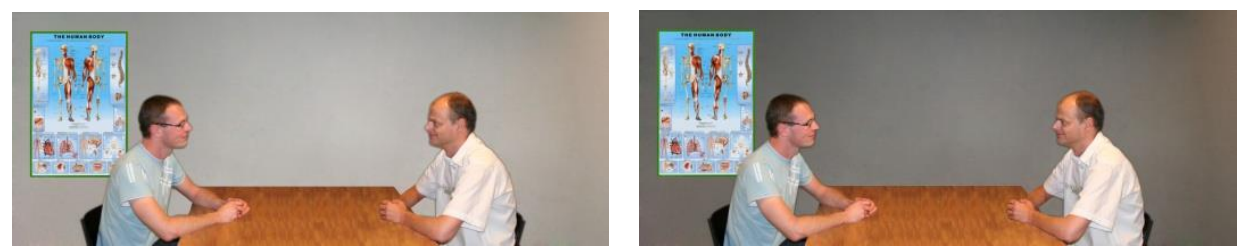

Figure 5.1. The two pictures of the different consultation rooms. Brightness is high for the left picture, and low for the right picture.

\section{Measures}

Responses to all scales were recorded on seven-point rating scales.

Perceived spaciousness. Perceived spaciousness was measured using the items: "I would feel constricted inside this room" (reverse coded), "I would feel confined inside this room" (reverse coded), "I would have sufficient freedom of movement inside this room", and "I would easily feel suffocated inside this room" (reverse coded) $(\alpha=.68)$.

Affective experience. To measure affective experience, a measure was used comprising the items: "Inside this room I would feel at ease", "Inside this room I would feel unhappy" (reverse coded), "I would feel uncomfortable inside this room" (reverse coded) and "This room would give me a pleasant feeling" ( $\alpha=.73)$. 
Intended self-disclosure. Intended self-disclosure was measured using the items: "I would feel inhibited from speaking inside this room" (reverse coded), "Inside this room I would feel able to speak freely", "I would feel uncomfortable in sharing personal information inside this room" (reverse coded) and "It would be hard for me to talk about myself inside this room" (reverse coded) $(\alpha=.82)$.

Liking. To measure the patient's judgment of the physician in terms of liking, a measure was used comprising the items: "This physician is unkind" (reverse coded), "This physician is involved", "This physician is empathetic" and "This physician is unfriendly" (reverse coded) $(\alpha=0.77)$.

\section{Results}

Analyses of variance (ANOVA) were conducted with perceived brightness and communicated threat as the independent variables and perceived spaciousness, affective experience and intended self-disclosure as the dependent variables. Results were analyzed for gender and age using analysis of variance, but since none proved significant there will be no further discussion of these variables $(p>$ .10 for all measures).

\section{Perceived spaciousness}

No main effect was found for perceived brightness $(F(1,88) 1.14, p=.289$, partial $\left.\eta^{2}=.01\right)$. Communicated threat, on the other hand, was found to have a significant effect on perceived spaciousness $\left(F(1,88)=4.52, p=.036\right.$, partial $\left.\eta^{2}=.05\right)$, indicating that the low threat condition triggered more perceived spaciousness than the high threat condition (Table 5.1).

Interestingly, and in line with expectations $(\mathrm{H} 2 \mathrm{a})$, an interaction was obtained between communicated threat and perceived brightness $(F(1,86)=4.43, p=$ .038, partial $\eta^{2}=.05$ ) (Figure 5.2). For the high threat condition, the difference in mean scores for perceived brightness was significant, with participants judging the brighter room as more spacious than the darker room $(F(1,86)=4.75, p=.032$, partial $\left.\eta^{2}=.05\right)$. For the low threat condition, this difference was not significant $(F<$ $1, n s$ ). Hence, perceived brightness only affected spaciousness perceptions (in the predicted direction) in the high threat condition. 


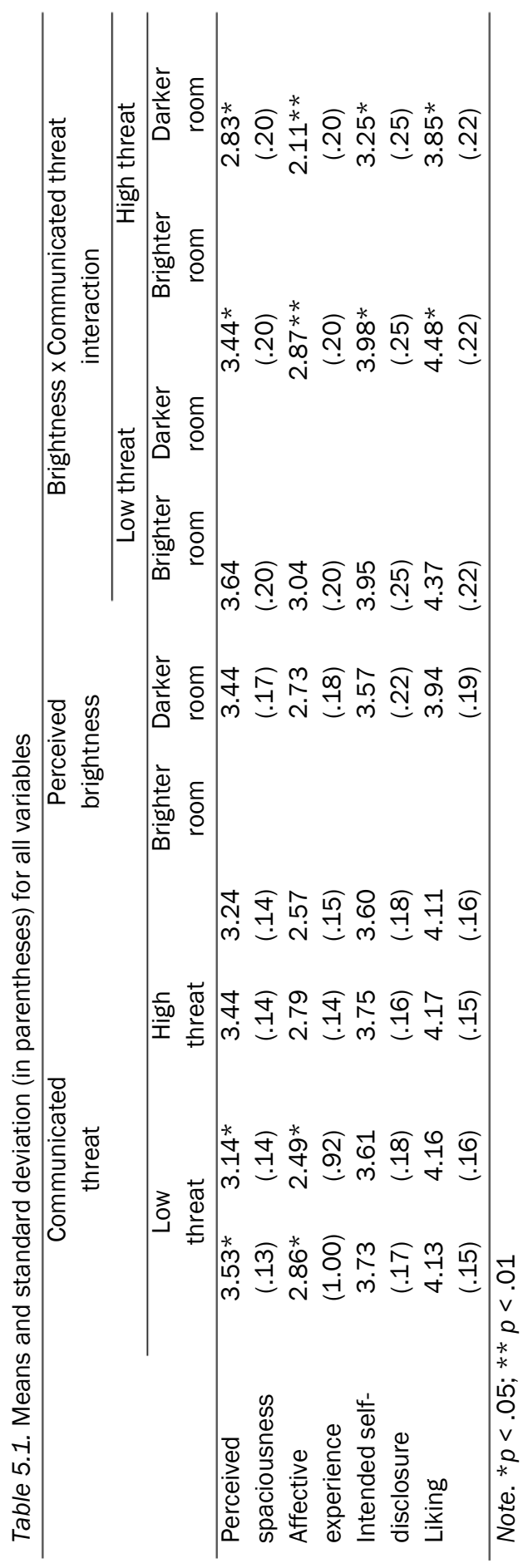




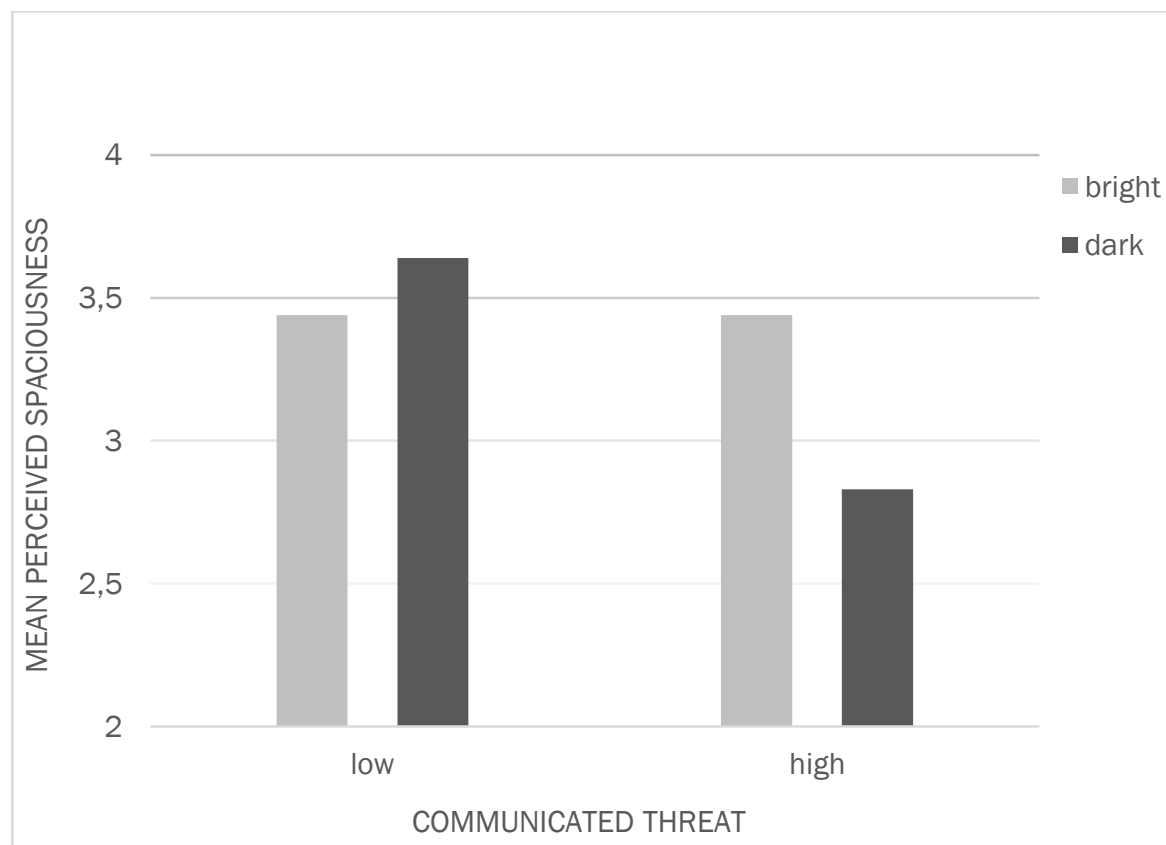

Figure 5.2. Interaction between brightness and communicated threat for perceived spaciousness scores.

Affective experience

Again, no main effect was found for perceived brightness $(H 1 b)(F(1,88)=1.35, p$ $=.249$, partial $\left.\eta^{2}=.01\right)$. Communicated threat, again, had a significant effect on the affective experience $\left(F(1,88)=4.02, p=.048\right.$, partial $\left.\eta^{2}=.05\right)$, indicating that the low threat condition generated more positive affect compared to the high threat condition.

Similar to the results for perceived spaciousness, an interaction was obtained between communicated threat and perceived brightness $(F(1,86)=7.30, p=$ .008 , partial $\eta^{2}=.08$ ) (Figure 5.3). For the high threat condition, the difference in mean affective experience scores for perceived brightness was significant, with participants experiencing more positive affect in the brighter room $(F(1,86)=7.04$, $p=.009$, partial $\left.\eta^{2}=.08\right)$. For the low threat condition, the difference in mean affective experience scores for perceived brightness was not significant $(F(1,86)=$ 1.26, $p=.264$, partial $\eta^{2}=.01$ ).

To test whether spaciousness perceptions can account for the latter interaction, analyses of covariance were conducted. Following the procedure of Baron and Kenny (1986) these analyses should show (in addition to yielding the effects described above) that the interaction effect between the two independent variables 
(perceived brightness and communicated threat) on the dependent variable (affective experience) should weaken when the mediator (perceived spaciousness) is included as a covariate in an ANCOVA. In addition, the effect of the mediator on the dependent variable should be significant. Analyses following these outlines show that the effect of the perceived brightness $x$ communicated threat interaction indeed becomes non-significant $\left(F(1,85)=3.53, p=.064\right.$, partial $\left.\eta^{2}=.04\right)$, while the influence of perceived spaciousness is significant $(F(1,85)=24.37, p<.001$, partial $\left.\eta^{2}=.22\right)$. In other words, in the high threat condition, the brighter room generated more positive affect because participants experience it as being more spacious.

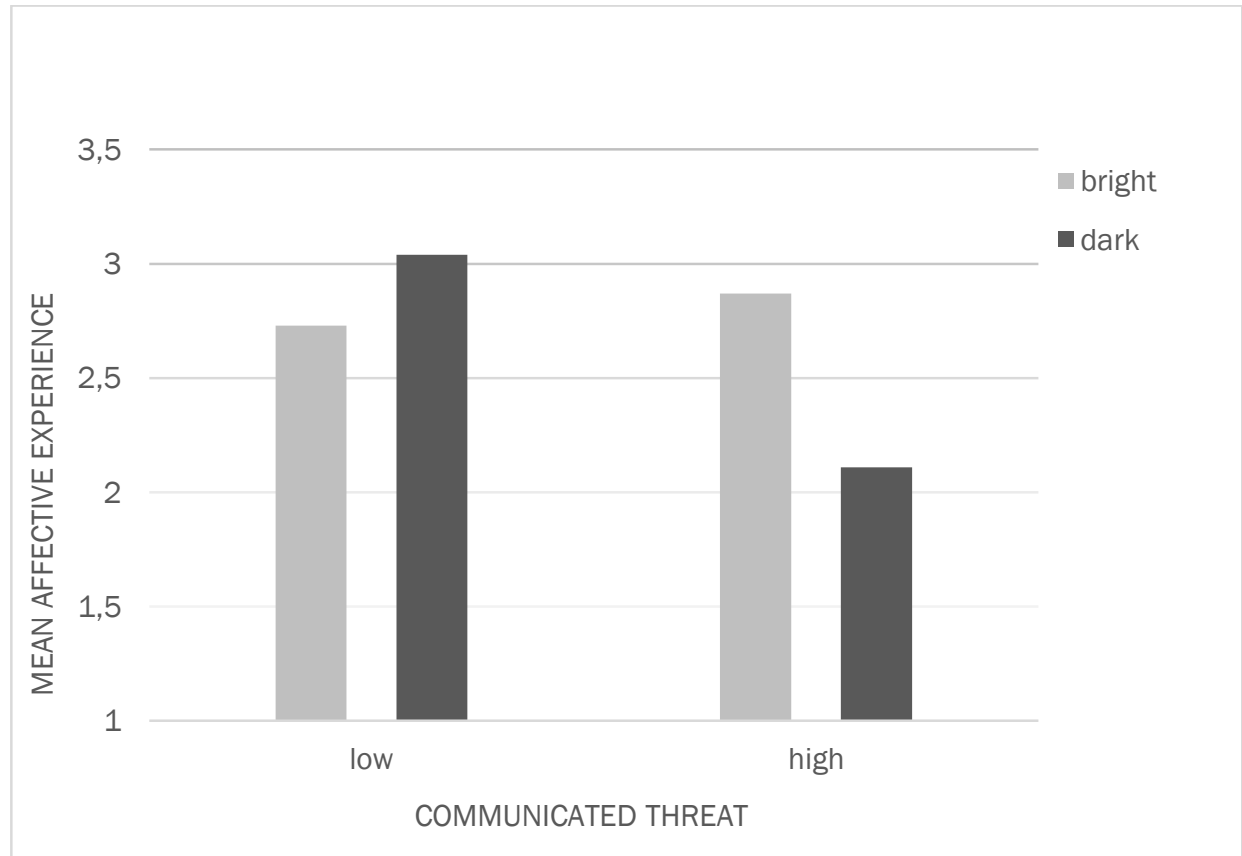

Figure 5.3. Interaction between brightness and communicated threat for affective experience scores.

Intended self-disclosure

No main effects were obtained for either room perceived brightness $(F<1, n s)$ or communicated threat $(F<1, n s)$. However, the interaction between communicated threat and perceived brightness was significant $(F(1,86)=5.45, p=.022$, partial $\eta^{2}=.06$ ) (Figure 5.4). Similar to the interactions above, in the high threat condition, the difference in mean scores for perceived brightness was significant, with participants having a higher intention to self-disclose in the brighter room $(F(1,86)$ $=4.37, p=.039$, partial $\left.\eta^{2}=.05\right)$. For the low threat condition, this difference was not significant $\left(F(1,86)=1.40, p=.240\right.$, partial $\left.\eta^{2}=.02\right)$. 


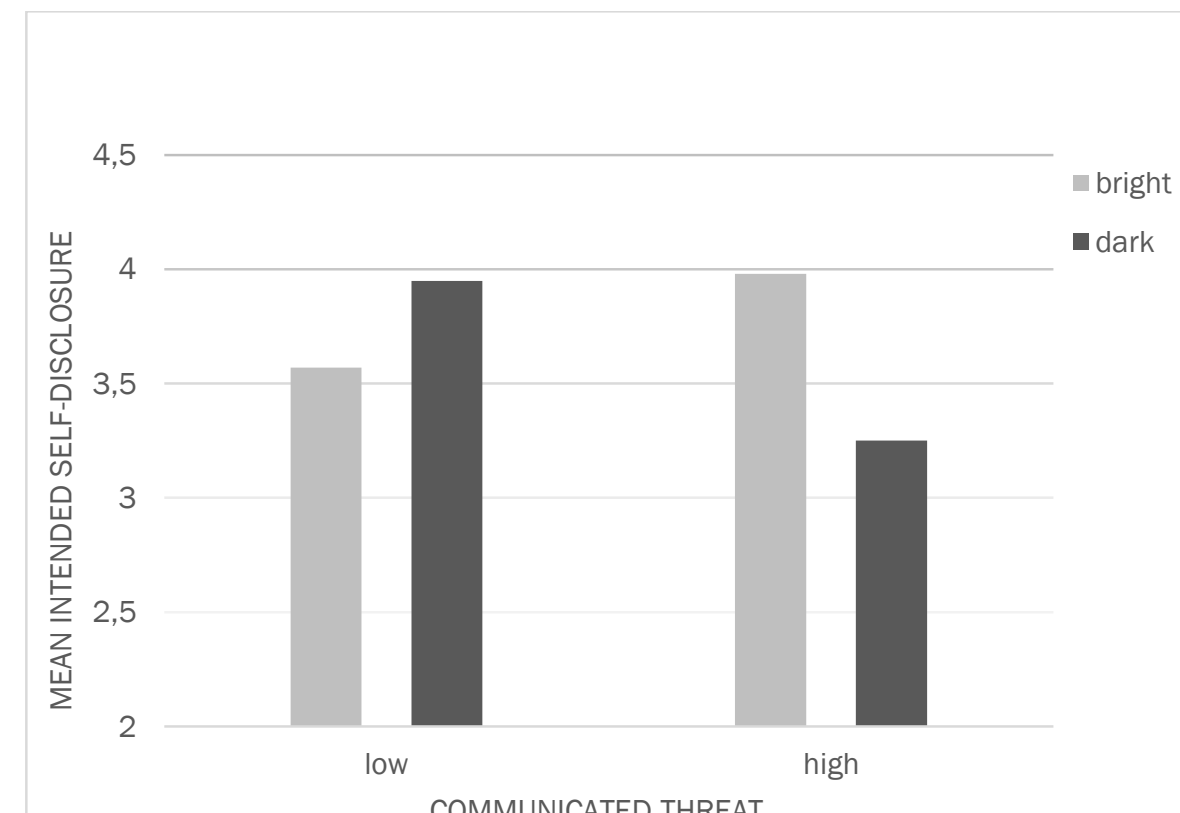

Figure 5.4. Interaction between brightness and communicated threat for intended selfdisclosure scores.

Again, we tested whether spaciousness perceptions underlie the obtained interaction between perceived brightness and communicated threat. When perceived spaciousness was included in the model, the influence of the perceived brightness $\mathrm{x}$ communicated threat interaction became non-significant $(F(1,85)=$ 2.76; $p=.100$, partial $\eta^{2}=.03$ ), while the influence of perceived spaciousness was significant $\left(F(1,85)=11.45 ; p=.001\right.$, partial $\left.\eta^{2}=.12\right)$. In other words, in the high threat condition, participants disclose more information in the brighter room because they experience more spaciousness.

Similar analyses were conducted to test whether the relationship between the perceived brightness $\mathrm{x}$ communicated threat interaction and intended selfdisclosure was mediated by affective experience. However, the mediating effect of this variable was non-significant $(p=.335)$.

\section{Liking}

No main effects were found for brightness $(F<1, n s)$ and communicated threat $(F<$ $1, n s)$. An interaction was obtained between communicated threat and brightness ( $F$ $(1,86)=6.42, p=.013$, partial $\eta^{2}=.07$ ) (Figure 5.5). For the high threat condition, the difference in mean scores for brightness was significant. With participants judging the physician more positive in the brighter room $(F(1,86)=4.26, p=.042$, 
partial $\left.\eta^{2}=.05\right)$. For the low threat condition, this difference was not significant ( $F$ $(1,86)=2.26, p=.136$, partial $\left.\eta^{2}=.03\right)$.

This time however, the effect of the brightness $x$ communicated threat interaction remained significant $\left(F(1,85)=4.56 ; p=.036\right.$, partial $\left.\eta^{2}=.05\right)$ when inserting perceived spaciousness as a covariate, and the influence of perceived spaciousness was non-significant $\left(F(1,85)=2.62 ; p=.110\right.$, partial $\left.\eta^{2}=.03\right)$. This indicates that the relationship between the room brightness $x$ communicated threat interaction and physician judgment is not mediated by perceived spaciousness.

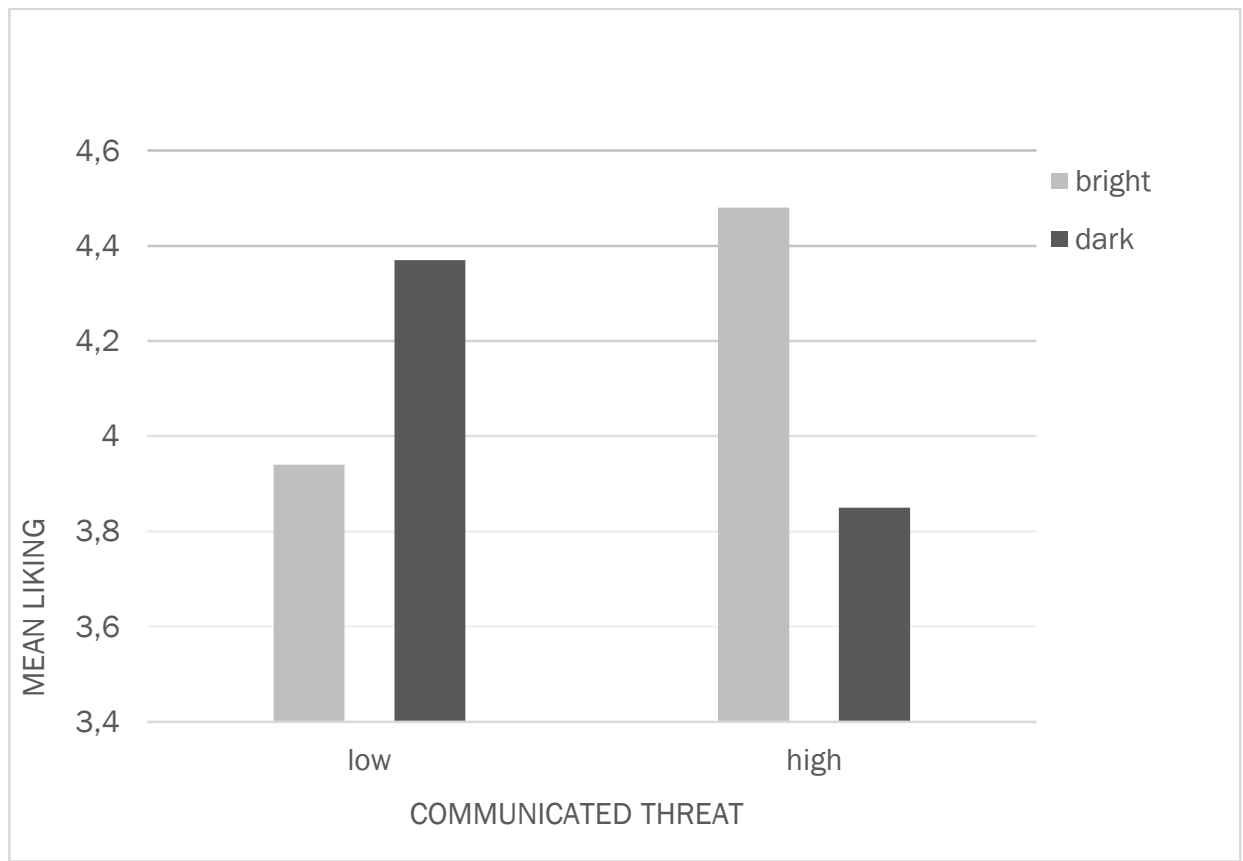

Figure 5.5. Interaction between brightness and communicated threat for liking.

It was examined whether the affective experience mediated the brightness $x$ communicated threat interaction. Insertion of the affective experience as a covariate revealed a significant effect of this mediator $(F(1,85)=6.19 ; p=.015$, partial $\left.\eta^{2}=.07\right)$. In addition, the brightness $x$ communicated threat interaction became non-significant $\left(F(1,85)=3.27 ; p=.074\right.$, partial $\left.\eta^{2}=.04\right)$, indicating that the relationship between room brightness $\mathrm{x}$ communicated threat and physician judgment is mediated by positive affect. In other words, in the high threat condition, participants judged the physician more positive in the brighter room because they experience more positive affect. 


\section{General discussion}

The results presented first and foremost show that effects of room atmospherics in the healthcare context vary depending on the patient's state of mind; no main effects were obtained for perceived brightness. However, when taking into account communicated threat, a relationship surfaced between perceived brightness and the outcome measures; in a threatening conversation, perceived brightness positively influenced perceptions of freedom, generated more positive affect, and a higher willingness to self-disclose. In a worry-free conversation, participants were unaffected by the brightness manipulation. These combined findings are in line with previous research indicating that people value more space particularly when they perceive the situation as threatening (Albas \& Albas, 1989; Dosey \& Meisels, 1969; Greene, 1977). In line with this emphasis on people's need for space, results further showed that the interactive effects of perceived brightness and communicated threat on both the affective experience and intended self-disclosure are mediated by perceived spaciousness.

In addition, the results revealed a significant main effect of communicated threat on perceived spaciousness. This finding provides strong evidence for the claim that environmental perception (and related affective and behavioral measures) is very much shaped by psychological circumstances; participants in the high threat condition actually perceived the room as less spacious compared to participants in the low threat condition. This finding suggests that depending on one's mindset, walls that appear at a safe and comforting distance in a joyous, relaxed situation may indeed seem to be 'closing in' when threat comes to the fore and anxiety takes over.

In line with reactance theory (e.g., Levav \& Zhu, 2009), our results show that a room that comes across as less spacious not only invokes less positive judgments, but also decreases self-disclose intentions. Hence, displaying a lower self-disclose intention can be seen as a form of reactance to a 'space invasion'. This is in line with results of Albert and Dabbs (1970), who studied the effect of interpersonal distance on attitude change. Their results show that when interpersonal distance decreases, the amount of reactance increases, transpiring in a lowered willingness to accept persuasive messages. Generally, reactance can be expected to surface in a refusal to comply with (implicit) requests, in our study the physician's 'request' to self-disclose information that allows for an accurate diagnosis and a fitting treatment or procedure.

Additionally, our results show that room brightness may also steer physician perceptions, a finding in line with previous research (e.g., Campbell, 1979; Maslow \& Mintz, 1956; Schiffenbauer \& Schiavo, 1976; Teven \& Comadena, 1996; Van 
Rompay \& Tanja-Dijkstra, 2010). Specifically, our results show that a brighter room makes the physician come across as more likeable. This relationship was not mediated by perceived spaciousness, but rather by the affective experience. It should be noted however that the mediation analyses presented across the variables by no means rule out additional mediators (especially when taking into account that the interaction terms remained marginally significant after insertion of the mediator as a covariate). For instance, earlier research suggests that prototypicality plays an important role in determining likeability-outcomes, with physicians displayed in more prototypical offices coming across as more positive (Swan, Richardson \& Hutton, 2003; Ward, Bitner \& Barnes, 1992). Alternatively, brightness may induce competence perceptions, perhaps generating more trust and hence more self-disclosure.

\section{Limitations}

The main limitation of the current study is that visual displays were used to represent the environments and that participants were not physically 'submerged' in an actual setting. However, the use of photographic material in environmental research has been shown to accurately simulate real environments (Bateson \& Hui, 1992; Hendrick, Martyniuk, Spencer \& Flynn, 1977; Stamps, 1990). A metaanalysis of research using both measurements obtained in actual environments and measurements obtained through photographic material revealed a 0.86 correlation (Stamps, 1990). Likewise, Okken, Van Rompay and Pruyn (2012) showed that reactions to limited space did not differ across simulated settings (i.e., photographic material) and actual environments. Of course, in order to enhance applicability of our findings and to allow recommendations on specific brightness levels (i.e., absolute values as opposed to relative differences in brightness levels), follow up studies are required.

Another point of attention is that in the current study no actual patients participated. Although one can safely assume that the students in our study have experiences visiting a general practitioner or specialist (and can draw on these encounters to imagine themselves in the scenarios described in our study), field studies examining actual behaviors of patients with actual (situation-specific) fears and worries in real environments are needed to further increase knowledge about the influence of environmental factors. Furthermore, although analyses did not reveal age and gender-related differences, it should be noted that participants in our study were all students similar in age, cultural background and education. Hence, our findings do not rule out that differences related to these factors play a role with different, or less homogeneous, target groups. For instance, concerning cultural background, Hofstede and colleagues have extensively documented differences across cultures with respect to variables such as power distance (i.e., the degree to which less powerful members of a society accept and expect that 
power is distributed unequally) and masculinity-femininity (e.g., Hofstede, Hofstede and Minkov, 2010). For instance, the masculinity side of the latter dimension represents a preference in society for achievement, and assertiveness and thus reflects the extent to which society at large is competitive. Its opposite, femininity, stands for a preference for cooperation and caring, and this reflects a more consensus-oriented society. Arguably, self-disclosure comes more natural and easy in the latter type of society, similar to how, on an individual level, self-disclosure is sometimes said to come easier for women (Dindia \& Allen, 1992).

Furthermore, although the results presented in this paper indicate that relatively small differences in perceived brightness influence both affective experiences and behavioral intentions, no conclusions can be drawn about specific brightness settings (apart from the obvious prediction that extreme values are likely to induce negative effects).

\section{Implications for Future Research and Practice}

Our findings show that altering the atmospherics can be used as a tool to improve the affective quality of the environment. In small environments in particular, perceived spaciousness can be increased by increasing room brightness (as our findings suggest). In addition to room brightness, previous research showed that lighting may likewise affect self-disclosure intentions (although the line of reasoning proposed in this paper has not been tested in relation to lighting) (Gifford, 1988). In addition to such atmospheric (non-tangible) variables, research suggests that material aspects of built environments may also affect spaciousness perceptions and can thus be used to improve the spatial ambiance of the environment. For instance, Stamps and Krishnan (2006) investigated the influence of wall texture or roughness on perceived spaciousness and showed that spaciousness perceptions differed across (otherwise identical) rooms varying in wall texture. Finally, environmental features such as furniture selection and positioning (i.e., layout) within the room can also affect spaciousness perceptions (e.g., with a greater interpersonal distance as the resultant of furniture layout enhancing spaciousness impressions and hence self-disclosure intentions).

Regardless of the environmental factor under discussion, however, it is most important to realize that such effects are very much dependent on the patient's state of mind. It could even be argued that as familiarity and intimacy with a physician increase in the course of a treatment, a more intimate setting might even generate positive effects, as also suggested by research showing that dim lighting may increase self-disclosure (Miwa \& Hanyu, 2006). And although effects of perceived brightness were non-significant in the low-threat conversation, the results across the dependent variables (see Figure 5.2) tentatively suggest that patients may prefer a less spacious setting when emotions such as relieve and happiness, 
rather than anxiety and fear, take over. Arguably then, physicians might benefit from means that allow for flexible adaptation of room atmospherics. For instance, usage of a dimmer switch in consultation rooms could enable the physician to adjust lighting conditions to the type of conversation at hand, using brighter lighting when high anxiety or stress levels can be expected (i.e., first visits, discussing results of medical tests, etc) and dimmed lighting for low-stress situations.

Based on the observation that such adjustments are particularly called for when patients face worries and anxiety, and that such a state of mind is common in many healthcare environments (e.g., visits to one's physician are usually not stress-free), our findings are particularly relevant in the healthcare context. Their importance is further stressed by research demonstrating that more active participation of patients during interactions with physicians (implying more self-disclosure) improves the effectiveness of medical consultations (Zandbelt, Smet, Oort, Godfried \& De Haes, 2007), and that patient satisfaction, adherence and medical outcomes fare well by increased self-disclosure (Harrington, Noble \& Newman, 2004). These combined findings underline the importance of attending to patients' affective needs and creating a soothing environment. Additionally, this type of knowledge can also be put to use in other types of services in relation to which creating a pleasant (service) environment is considered important. This also follows from research examining effects of spatial density and experienced spaciousness in retail environments, showing that creating open spaces (Haytko \& Baker, 2004; Van Rompay, Galetzka, Pruyn \& Moreno-Garcia, 2008) may boost shopping satisfaction.

Awaiting future research addressing these and related issues, the findings presented are a first step towards unraveling how environmental and psychological variables conjointly influence affective experiences and related behaviors. 
Chapter 5 demonstrates that, besides tactile factors on architectural and interior design level, room atmospherics are also able to influence spaciousness perceptions. Moreover, the results shed more light on the impact of room brightness on our affective experience and communicative behavior. Chapter 6 examines the impact of room brightness on online self-disclosure (study 6.2). In addition, the two studies reported investigate whether online self-disclosure is affected by on-screen spaciousness perceptions, which was varied by manipulating website design. The results of the studies show that spaciousness perceptions activated by both the physical environment and website design positively influence self-disclosure. Furthermore, it was shown that negative influences stemming from the physical environment (i.e., room brightness), over which online service providers have little to no control, may be counteracted or at least reduced by the use of a more spacious website design. 


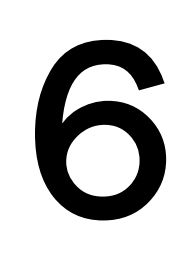

A spacious view: Influences of the physical
and online environment on self-disclosure 
"Happiness can be found, even in the darkest of times, if one only remembers to turn on the light."

(Albus Dumbledore in Harry Potter and the prisoner of Azkaban, J. K. Rowling,

1999)

\section{Introduction}

The process of communicating personal information about oneself to another individual is called self-disclosure (Strassberg, Roback, D'Antonio \& Gabel, 1977). Self-disclosure is of importance in many different service environments. Whether talking to a physician, meeting a wedding planner, getting a mortgage on your house or going through a loved one's final wishes with a mortician, information disclosure by consumers is a prerequisite for optimized service delivery. During such dyadic conversations, there are many factors that influence self-disclosure levels such as consumer traits (e.g., shyness, introversion, reservedness), the conversation itself (e.g., sensitive topics, level of reciprocity), and the environment in which the conversation takes place (Cozby, 1973; Ignatius \& Kokkonen, 2007; Okken, Van Rompay \& Pruyn, 2012; Omarzu, 2000). Of special importance to current undertaking is the relationship between spaciousness perceptions and selfdisclosure, with previous research suggesting that physical space may generate psychological space, subsequently enhancing self-disclosure (Okken et al., 2012).

In addition to face-to-face communication, the internet provides us with a virtual environment in which we can interact with others: the online environment. New websites that offer ways to interact with other internet users (i.e., social network sites, forums, and blogs) emerge on a daily basis. Besides offering an easy way to stay in touch with friends and family, the internet is also a territory well explored by service providers. With this trend, the amount of self-disclosure on the internet is steadily increasing. For instance, online consults with a doctor or counselor, interaction with other forum members, and filling out extensive profiles on online dating sites are nowadays common activities. While self-disclosure often occurs in verbal form during dyadic or group conversations, writing or typing personal information (with the intention to 'share' with others) is also a type of self-disclosure (Ignatius \& Kokkonen, 2007), and both types have been used in self-disclosure research (Barak \& Gluck-Ofri, 2007; Gifford, 1988; Joinson, 2001; Joinson, Paine, Buchanan \& Reips, 2008; Jourard \& Friedman, 1970). 
Although the impact of environmental factors on self-disclosure during face-to-face communication is well established (Jourard \& Friedman, 1970; Gifford, 1988; Miwa \& Hanyu, 2006; Okken, Van Rompay \& Pruyn, 2012; Sundstrom, 1975), it is an open question whether environmental factors that influence self-disclosure during face-to-face interaction, can also influence online communication. In addition, spaciousness perceptions may be activated through website design. For instance, similar to how interpersonal distance between conversation partners and room brightness may influence self-disclosure in face-to-face consults (Gifford, 1988; Miwa \& Hanyu, 2006), these factors also figure in the online environment (e.g., screen brightness and perceived distance of an online service provider).

The foregoing suggests that spaciousness perceptions might ensue from both the physical and the online environment (i.e., the computer screen). However, the latter prediction has not been tested so far. Hence, in study 6.1, we will first test whether spaciousness perceptions activated through website design can influence selfdisclosure. In study 6.2, the interaction between both types of factors will be explored. To this end, both room brightness and website design were manipulated in order to study effects on actual disclosing behaviors and affective experiences of participants during computer-mediated social interaction. Before presenting the details of these studies, first a brief overview of relevant research is presented next.

\section{Environmental influences and self-disclosure}

Research shows that environmental factors such as room architecture and interior design factors such as desk size can be used to stimulate or hinder self-disclosing behavior (Okken, Van Rompay \& Pruyn, 2012; Sundstrom, 1975). Furthermore, room atmospherics have been shown to influence disclosing tendencies in simulated environments (Gifford, 1988; Miwa \& Hanyu, 2006). One possible explanation of why the environment influences our feelings and behavior holds that creating a comfortable or pleasant environment can facilitate self-disclosure, arguably because such environments make people feel comfortable and at ease, which in turn increases disclosure tendencies (Chaikin, Derlega \&Miller, 1976; Gifford, 1988; Miwa \& Hanyu, 2006). For instance, Gifford (1988) studied the effects of lighting and room decor on interpersonal communication. His results show that brighter lighting and a more home-like decor made respondents feel more comfortable, which in turn stimulated both general and intimate communication.

However, creating a pleasant environment can be considered challenging when taking into account that experienced pleasure is determined by several different 
factors inside the room, ranging from large-scale furniture objects to small decorative or personal objects (Verhoeven, Van Rompay \& Pruyn, 2007). In addition, pleasure is subject to individual differences and largely depends on personal taste and distaste of, for instance, different decorative styles and color schemes, making it hard to pinpoint which factors within an environment should be attended to. In this paper it is argued that environmental factors affect selfdisclosure via experienced spaciousness, a more straightforward concept that has also been shown to influence self-disclosure in the health context (cf. Okken, Van Rompay \& Pruyn, 2012; Sundstrom, 1975).

\section{Spaciousness and the physical environment}

Sundstrom (1975) studied the effects of room size and showed that limited space can induce crowding perceptions, which in turn may decrease the willingness to communicate. In addition, Okken, Van Rompay and Pruyn (2012) investigated the effects of both room size and interpersonal distance on self-disclosure intentions and affective experiences in experiments using pictures of a simulated patient consult with a general practitioner. The results of these experiments show that both increases in room size and desk size (i.e., interpersonal distance) may positively influence self-disclosure tendencies and affective experiences (i.e., experienced pleasantness of the disclosure episode), and that these relationships are mediated by experienced spaciousness. These findings suggest that increases in physical space generate feelings of enhanced psychological space, which in turn induce selfdisclosure and create a more positive affective experience.

A deeper understanding of how such spaciousness perceptions influence behavior follows from a study by Meyers-Levy and Zhu (2007), who investigated the effects of ceiling height. They showed that a heightened ceiling (i.e., a more spacious setting) activates feelings of freedom, which in turn generates more creative strategies in problem-solving tasks. In a similar vein, Levav and Zhu (2009) examined the effects of experienced spaciousness in store environments. Their results showed that reducing the width of shopping aisles activated feelings of confinement, and that these negative feelings led consumers to restore 'freedom' (i.e., reactance) by making more varied product choices (which can be seen as an act / expression of freedom in western societies). These findings suggest that restraining physical space indeed invokes feelings of limited psychological space (cf. Okken, Van Rompay \& Pruyn, 2012), which is in line with embodiment research showing that spatial properties can frame people's reasoning about more abstract, mental concepts (IJzerman \& Semin, 2010; Williams \& Bargh, 2008). 
In addition to variations related to the actual measurements and physical parameters of the environment, spaciousness perceptions may also vary with room atmospherics such as color. For instance, Acking and Küller (1972), who repainted dayrooms in a hospital to investigate the influence of wall color, found that a white room was judged as most open, compared to a light-green and a dark-green room. Comparable results were found by Kwallek (1996), who studied the influence of color in an office environment and showed that a white wall color (in comparison to green and red wall colors) received the highest spaciousness scores. Furthermore, results of Oberfeld, Hecht and Gamer (2010) showed that not only a brighter wall color, but also a brighter ceiling color positively affected experienced spaciousness.

Besides color schemes, lighting conditions have also been shown to affect spaciousness perceptions. For example, Flynn, Spencer, Martyniuk and Hendrick (1973) showed that spaciousness judgments differed significantly for rooms with varying lighting conditions. Their results showed that lighting all four walls (as compared with overhead lighting that merely lights the center of the room) induced greater feelings of spaciousness. In line with these findings, Durak, Olgonturk, Yener, Guvenc and Gurcinar (2007) found that brighter room lighting conditions were judged as more spacious. Finally, Manav (2007) investigated lighting conditions in an office setting and showed that brighter lighting conditions yielded higher scores for both comfort and spaciousness compared to more dimmed conditions. When considering these effects, the question rises to what extent spaciousness perceptions play a role in online environments.

\section{Spaciousness and the online environment}

Although the influence of website design on self-disclosing behavior is an unexplored research area, results from online consumer research provide evidence that website design can impact emotions, attitudes and behavior (Dailey, 2004; Eroglu, Machleit \& Davis, 2001; Kim, Fiore \& Lee, 2007; Koo \& Ju, 2010; Mandel \& Johnson, 2002; Van Rompay, De Vries \& Van Venrooij, 2010). For instance, Dailey (2004) proposed that web design elements that threaten consumers' feelings of control inspire psychological reactance and in turn lead to negative emotions and attitudes towards the website and site avoidance behavior. In addition, Koo and Ju (2010) examined the effect of website design on emotions and online shopping intentions. Their results showed that the use of graphics and more distinctive colors on websites may evoke more pleasure and arousal compared to less use of graphics and colors. Furthermore, research on print advertising shows that white space is used in advertisements to increase attention and focus on product and the brand name. Interestingly, however, results show that the use of white space may 
also lead to more positive consumer evaluations (Olson, Pracejus \& O'Guinn, 2012; Pracejus, Olsen \& O'Guinn, 2006; Strong, 1926), presumably because they trigger spaciousness perceptions.

Foregoing suggests that in addition to impacting online shopping behaviors, website design may also influence self-disclosing behavior during an online disclosure task. Based on results of research examining environmental influences on self-disclosure during face-to-face interaction, we expect that website design elements such as screen brightness, website layout and perceptions of interpersonal distance onscreen may likewise impact spaciousness perceptions, and in turn self-disclosure, during online interaction. Building on findings showing that interpersonal distance impacts experienced spaciousness (cf. Okken, Van Rompay \& Pruyn, 2012; Sundstrom, 1975), in study 6.1, we hypothesize that a more spacious website design can be created by manipulating the perceived distance between service provider and the webcam. A larger perceived distance will increase spaciousness perceptions, in turn leading to a more positive experience and generating more selfdisclosure. Conversely, a smaller interpersonal distance (i.e., a service provider presented more 'up close' on the screen) will bring about the impression of a less spacious, more restricting website design, decreasing spaciousness perceptions and in turn limiting self-disclosure and creating negative affect. Hence:

H1A: A larger perceived distance will induce more spaciousness, thereby generating more self-disclosure than a smaller perceived distance.

H1B: A larger perceived distance will induce more spaciousness, thereby creating a more positive experience than a smaller perceived distance.

In addition, based on findings showing that atmospherics such as color and lighting can impact spaciousness perceptions (cf. Flynn, Spencer, Martyniuk \& Hendrick, 1973; Kwallek, 1996; Manav, 2007; Oberfeld, Hecht \& Gamer, 2010), it is predicted that website spaciousness also varies as a function of screen brightness. A brighter background will increase spaciousness perceptions, in turn leading to a more positive experience and generating more self-disclosure. Conversely, a darker background will decrease spaciousness perceptions, in turn leading to a less positive experience and to less self-disclosure. Hence:

H2A: A brighter background will induce more spaciousness, thereby generating more self-disclosure than a darker background.

H2B: A brighter background will induce more spaciousness, thereby creating a more positive experience than a darker background.

Furthermore, research suggests that the need for more spacious surroundings is higher in stressful situations than in stress-free situations (Dosey \& Meisels, 1969; 
Greene, 1977; Okken, Van Rompay \& Pruyn, 2012; Schiffenbauer \& Schiavo, 1976). For instance, results of Schiffenbauer and Schiavo (1976) show that during negative conversations a larger interpersonal distance induces more likeability than a smaller interpersonal distance, whereas during positive conversations, a smaller interpersonal distance yields a more positive response. This is in line with findings of Okken, Van Rompay and Pruyn (2012), showing that the need for space is less pronounced during a positive conversation, as opposed to a negative conversation. Based on these findings we expect that a negatively toned conversation is perceived as more threatening, either because the negative information consists of critique (Greene, 1977; Schiffenbauer \& Schiavo, 1976) or because it presents a personal risk (Okken, Van Rompay \& Pruyn, 2012). Therefore we hypothesize that when a more serious threat is introduced, the need for spaciousness will be higher than when the threat is less serious. Hence:

H3A: The effects of the perceived distance on the amount of self-disclosure and affective experience will be more pronounced when the communicated threat is low, as opposed to high.

$H 3 B$ : The effects of the background brightness on the amount of self-disclosure and affective experience will be more pronounced when the communicated threat is low, as opposed to high.

To test the hypotheses outlined, an online disclosure task was constructed consisting of an artificial online consult with the general practitioner's office of the local university. During this online consult perceived spaciousness was manipulated by altering the interpersonal distance (i.e., the size of the depicted person) and the brightness of the background of the screen. In addition, during the introduction of the experiment, the level of communicated threat was manipulated. This resulted in a 2 (interpersonal distance: small versus large) by 2 (background brightness: high versus low) by 2 (communicated threat: high versus low) between subjects design.

\section{Study 6.1}

A total of 93 participants (40 male and 53 female; mean age 21.72 years, SD = 2.26) participated in the experiment. The participants were all (under)graduate students of a Dutch university. At the start of the experiment, participants were informed that the purpose of the study was to extend knowledge about students' lifestyle and coping with stress. It was explained that the study was commissioned by the general practitioner's office of the university and also served as a trial to improve the online consult. Next, some practical information was given about how to answer the questions during the online consult. The layout of the online consult 
was roughly based on the layout of Windows Messenger, with a chat screen displaying the message of the other person, an interaction screen where the participants could enter their message and a picture of the other person (Figure 6.1). To make the chat more convincing, the screen also showed a status bar similar to the Windows Messenger program, containing messages such as "busy", "typing" and "connecting".

After the practical information was given, a button appeared to make a connection. Next, the picture of the employee of the general practitioners office appeared on the screen and an introduction appeared in the chat screen, which could be either neutral or high threat. After the introduction, a series of stress-related questions appeared one by one. Each question required an answer and pressing 'Enter'. After the last stress-related question, a final message appeared of the employee of the general practitioners office to thank the participant and explaining that pressing the button would disconnect them from the online consult and would forward them to a short evaluation of the new online consult layout. The evaluation consisted of seven-point scale items to measure perceived spaciousness, perceived ease of selfdisclosure and manipulation checks. After this short evaluation, participants were thanked for their participation and were free to leave.

\section{Independent variables}

The presented introduction to the experiment either stated that the survey was part of a large student lifestyle research project (neutral threat) or that the survey was conducted to investigate the prevalence and severity of stress and stress-related illnesses amongst students since there seems to be a high threat of stress-related illnesses for students (high threat). The scenario presented to the participants (translated from Dutch; manipulations are displayed in bold typeface; wording used in the high threat condition are between brackets) stated:

"Hello, my name is Lisanne and I work at the campus UT general practitioners office. As part of a periodical study I would like to ask you some questions about stress. It is very important for us to gain information about how students deal with feelings and stress. The study is part of an annual evaluation of lifestyle amongst our patients and the data will be saved in our database. (Recent results show that students are coping with much stress, which is bad for their health in the short term but especially in the long term. Stress can cause chronic diseases to the heart and arteries. Therefore, we find it important to gain information about how much stress students have to endure.) Your participation is anonymous. Before we continue, I would like to mention that answering the questions is not compulsory. Let's start with some short questions, ok?" 
Perceived spaciousness was manipulated in two ways; by altering the size of the person in the picture (i.e., interpersonal distance) and by altering the brightness of the background. In order to manipulate the interpersonal distance, a picture was used of a general practitioners office, in which a staff member was depicted. The size of the person in the picture was manipulated using Adobe Photoshop. A pilot study was conducted in which 15 participants were shown a series of photos with varying sizes of the depicted person. The participants were asked whether the picture was realistic as a screenshot taken with a webcam and to judge at what distance this person was sitting from her computer screen (and webcam). All questions were measured on seven-point Likert-type rating scales. Based on these results the two pictures were selected (see Figure 6.1) that received similar scores for realism $(t<1, n s)$ but differed for the judged distance $(M=2.27, S D=1.00$ vs. $M=5.60, S D=.91 ; t=9.75 ; d f=28 ; p<.000)$. During the experiment, the distance between the computer screen and the respondents was carefully monitored and kept constant.

In order to manipulate the brightness of the background of the screen, two different background colors were used. In order to create these different background colors, the brightness level was varied. A pilot study was conducted with 12 respondents, who were shown a series of screen backgrounds on a computer screen that differed for brightness. They were asked to judge the background brightness on a sevenpoint Likert-type rating scale and to indicate whether this background was suitable to use as a computer screen background. Based on these results, a dark (RGB = $146,175,100)$ and a bright $(R G B=146,175,200)$ background were chosen (see Figure 6.1) that differed significantly in brightness scores $(M=4.25, S D=1.14$ vs. $M=5.50, S D=1.00 ; t=2.86 ; d f=22 ; p=.009$ ) but received similar scores for suitability $(t<1, n s)$.

\section{Measures}

A seven-point Likert-type scale was used (1: "strongly disagree" to 7: "strongly agree") to measure the constructs.

\section{Self-disclosure.}

Self-disclosure was measured by analyzing the answers to the stress-related questions. The topics of the questions were: occurrence of stress, causes of stress, effects of stress, worrying about personal health and stress-reducing activities. There were two measures for self-disclosure: word count and the amount of topics mentioned during the answer. 

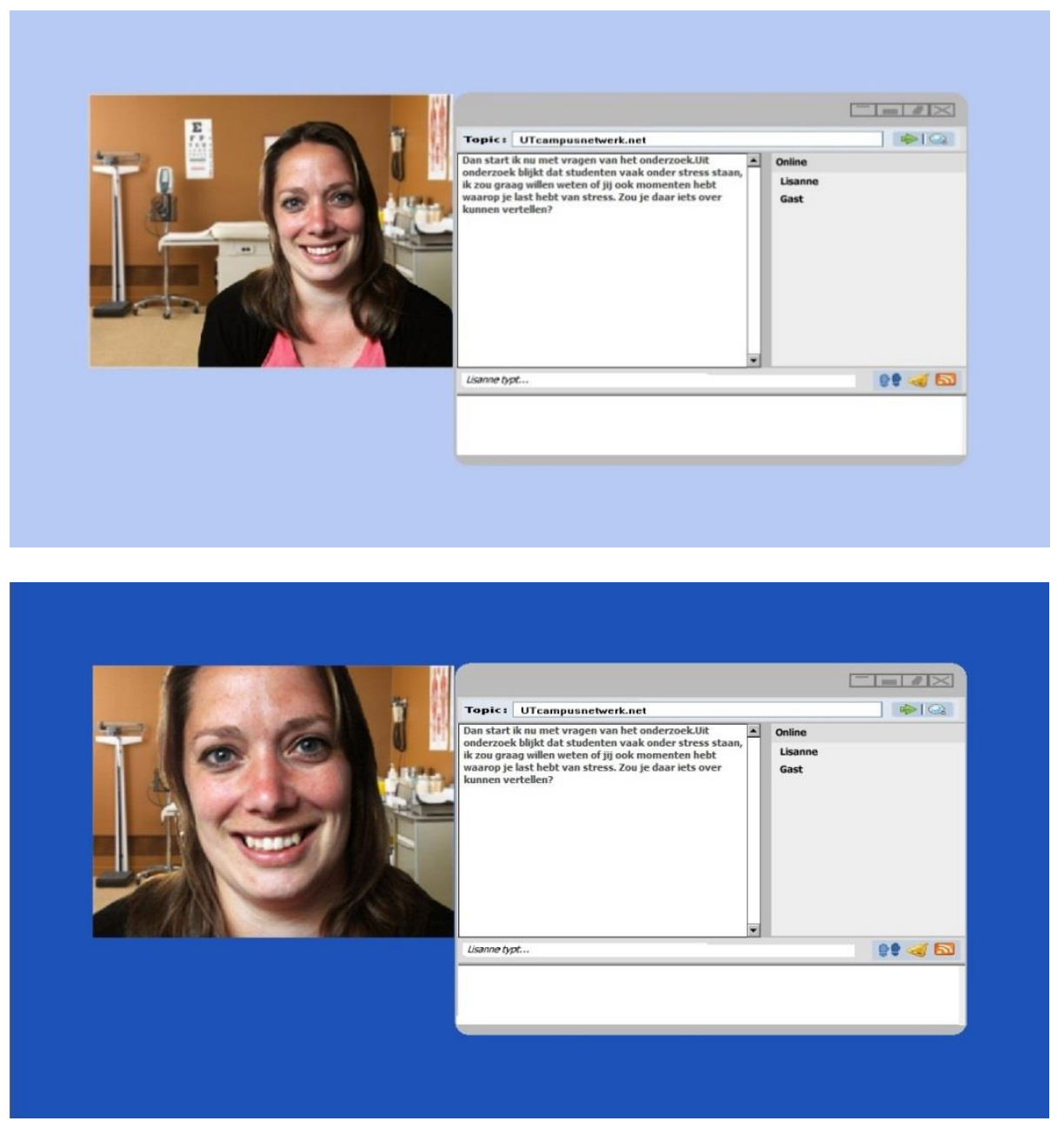

Figure 6.1. Screenshots of the experiment showing the manipulations of both background brightness and interpersonal distance. Background brightness is high for the upper (RGB = $146,175,200)$ and low for the lower picture $(R G B=146,175,100)$. Interpersonal distance is large for the upper picture and small for the lower picture.

\section{Perceived spaciousness.}

Perceived spaciousness was measured using the items: "I felt constricted inside this room" (reverse coded), "I felt confined inside this room" (reverse coded), "I had sufficient freedom of movement inside this room", and "I felt suffocated inside this room" (reverse coded) $(\alpha=.80)$. 
Affective experience.

To measure patient well-being, an affect-measure was used comprising the items: "Inside this room I felt at ease", "Inside this room I felt unhappy" (reverse coded), "I felt uncomfortable inside this room" (reverse coded), and "This room gave me a pleasant feeling" $(\alpha=.83)$.

Perceived ease of self-disclosure.

The perceived ease of self-disclosure was measured using the items: "I felt inhibited from speaking inside this room" (reverse coded), "Inside this room I felt able to speak freely", "I felt uncomfortable sharing personal information inside this room" (reverse coded), and "It would be hard for me to talk about myself inside this room" (reverse coded) $(\alpha=.86)$.

\section{Results}

A multivariate analysis of variance (MANOVA) was conducted with background brightness, interpersonal distance and communicated threat as independent variables, and with perceived spaciousness, affective experience, perceived ease of self-disclosure, word count and amount of topics as dependent variables. The analysis yielded a significant effect of background brightness $(F(5,81)=3.24, p=$ .010 , partial $\left.\eta^{2}=.16\right)$. Also, the multivariate effect of the interaction between background brightness and communicated threat reached significance $(F(5,81)=$ 2.60, $p=.031$, partial $\eta^{2}=.14$ ). In addition, the multivariate effect of the three-way interaction between background brightness, interpersonal distance and communicated threat was significant $\left(F(5,81)=3.07, p=.014\right.$, partial $\left.\eta^{2}=.16\right)$. Having established these effects, next the ANOVAs (comprising the same independent and dependent variables) are presented. An overview of all means and standard deviations of the found effects is given in Table 6.1. 


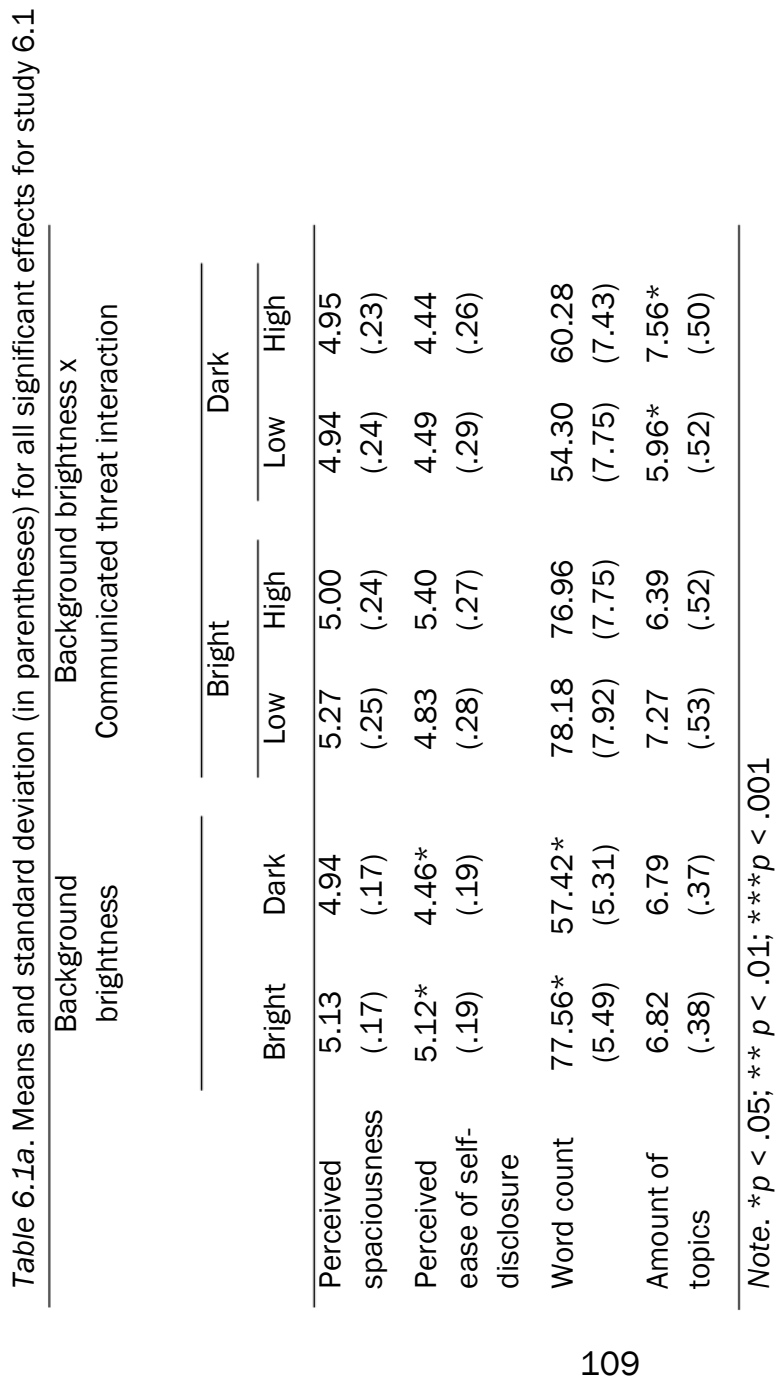




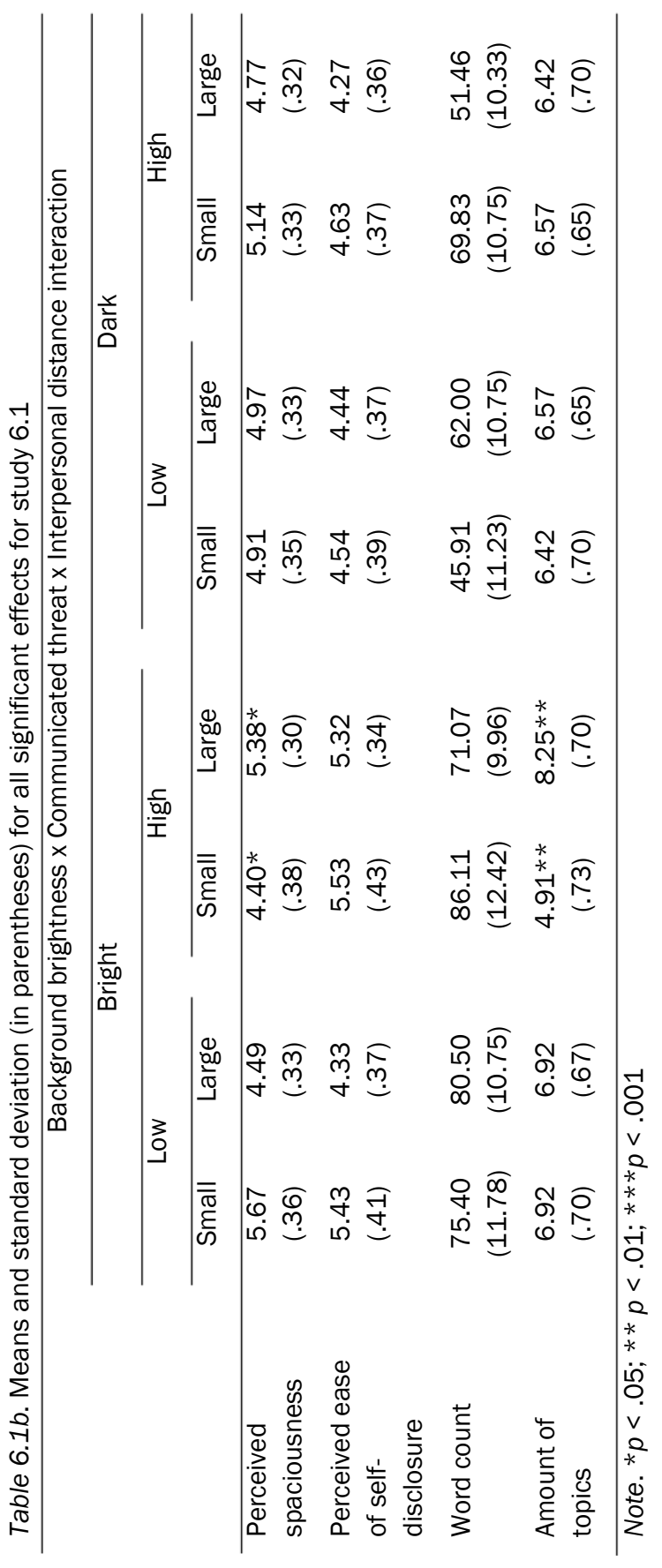




\section{Perceived spaciousness}

No main effects or two-way interactions were found for perceived spaciousness. A three-way interaction was obtained between background brightness, interpersonal distance and communicated threat $\left(F(1,85)=4.90, p=.030\right.$, partial $\eta^{2}=.06$ ) (see Figure 6.2). For the bright background, a significant effect was found for the high threat condition; the large interpersonal distance yielded higher spaciousness scores than the small interpersonal distance (large distance $M=5.38, S D=.30$ vs. small distance $M=4.40, S D=.38 ; F(1,85)=3.93, p=.049$, partial $\left.\eta^{2}=.05\right)$. For the low threat condition, the difference did not reach significance (large distance $M$ $=4.94, S D=.33$ vs. small distance $M=5.67, S D=.36 ; F(1,85)=2.16, p=.146$, partial $\left.\eta^{2}=.03\right)$.

For the dark background, no significant effects were obtained for perceived spaciousness (For the low threat condition: $M=4.97, S D=.33$ vs. $M=4.91, S D=$ $.35 ; F<1$, ns. For the high threat condition: $M=4.77, S D=.32$ vs. $M=5.14, S D=$ $.33 ; F<1, n s)$

\section{Perceived ease of self-disclosure}

Background brightness had a significant effect on the perceived ease of selfdisclosure $\left(F(1,91)=6.06, p=.016\right.$, partial $\left.\eta^{2}=.06\right)$. The bright background yielded higher scores for the ease of self-disclosure $(M=5.12, S D=.19)$ in comparison to the dark background $(M=4.46, S D=.19)$.

\section{Self-disclosure}

Word count. Background brightness had an effect on the overall word count ( $F(1$, $91)=6.95, p=.010$, partial $\left.\eta^{2}=.07\right)$. Over all answers, the amount of words used was higher in the condition with the bright background $(M=77.56, S D=5.49)$ compared to the condition with the dark background $(M=57.42, S D=5.31)$. 


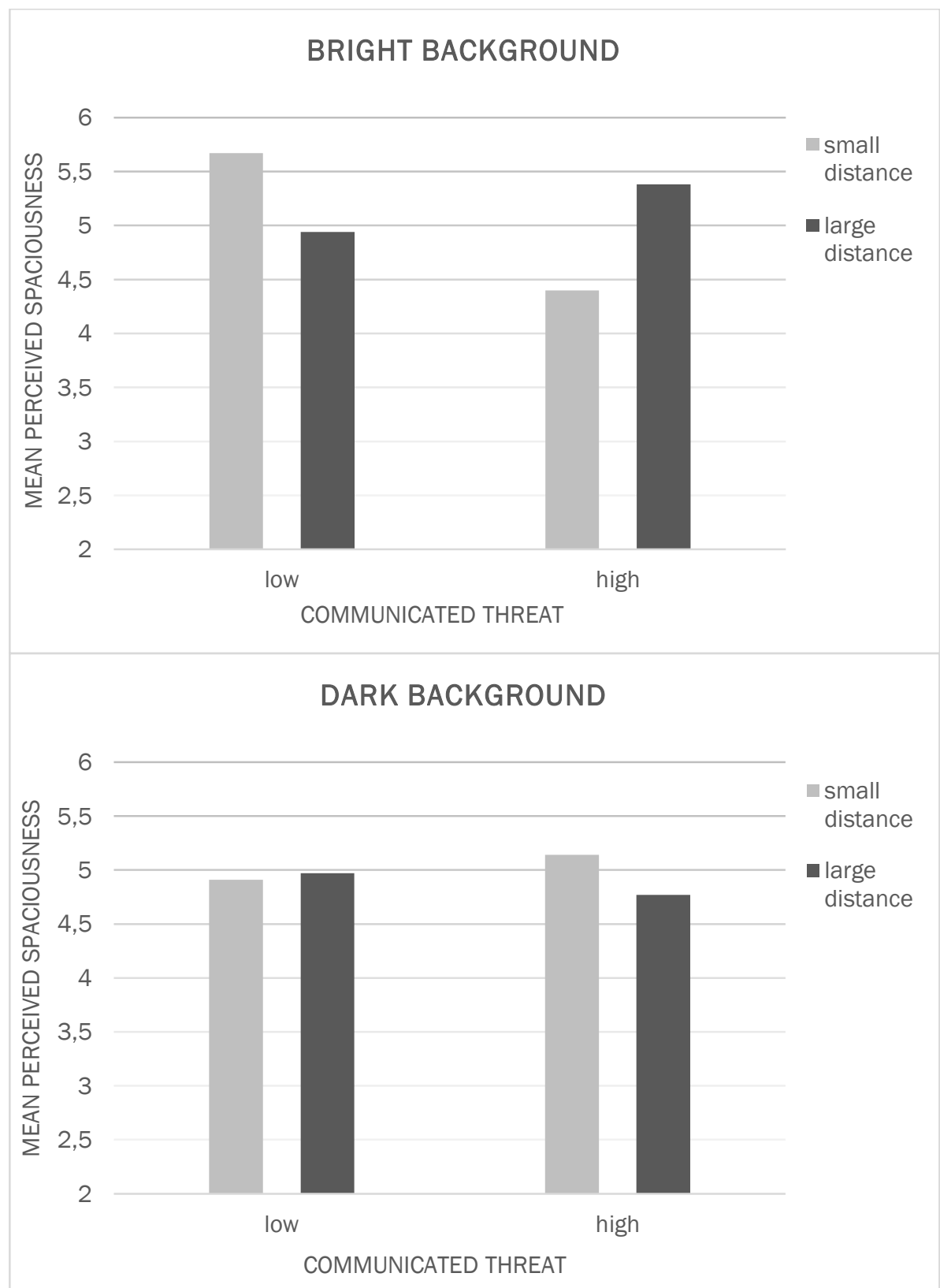

Figure 6.2. Three-way interaction between background brightness, communicated threat and interpersonal distance for perceived spaciousness, with the upper graph showing the bright background and the lower graph showing the dark background. 
Amount of topics. For the amount of topics, a significant interaction was obtained between background brightness and communicated threat $(F(1,89)=5.76, p=$ .018 , partial $\left.\eta^{2}=.06\right)$. For the dark background, there was a significant difference for communicated threat (low threat $M=5.96, S D=.52$ vs. high threat $M=7.56$, $S D=.50 ; F(1,89)=4.96, p=.029$, partial $\left.\eta^{2}=.05\right)$. For the light background, this difference did not reach significance (low threat $M=7.27 S D=.53$ vs. high threat $M=6.39 S D=.52 ; F(1,89)=1.41, p=.239$, partial $\left.\eta^{2}=.02\right)$. However, this interaction was qualified by a three-way interaction $(F(1,85)=7.85, p=.006$, partial $\eta^{2}=.09$ ) (see Figure 6.3). For the bright background, a significant effect was found for the high threat condition; in the large interpersonal distance condition more different topics were discussed than in the small interpersonal distance (large distance $M=8.25, S D=.70$ vs. small distance $M=4.91, S D=.73 ; F(1,85)=$ 10.93, $p=.001$, partial $\eta^{2}=.11$ ). For the low threat condition (see Figure 6.3, bottom panel), the difference did not reach significance (large distance $M=6.92$, $S D=.67$ vs. small distance $M=6.92, S D=.70 ; F<1, n s)$. For the dark background, no significant effects were obtained for the number of topics (For the low threat condition: small distance $M=6.42, S D=.70$ vs. large distance $M=6.57$, $S D=.65 ; F<1$, ns. For the high threat condition: $M=6.57, S D=.65$ vs. $M=6.42$, $S D=.70 ; F(1,85)=3.87, p=.05$, partial $\left.\eta^{2}=.03\right)$

\section{Discussion}

The results show that both perceived spaciousness and the self-disclosure measures are affected by the on-screen manipulations. The results largely show that a more spacious website design stimulates more self-disclosure compared to a less spacious or more confining design. This is in line with findings of research examining effect of physical environmental manipulations such as interpersonal distance (Jourard \& Friedman, 1970; Okken, Van Rompay \& Pruyn, 2012) and lighting conditions (Gifford, 1988) on self-disclosure.

In line with expectations, the results suggest that spaciousness perceptions take on a more important role under high threat circumstances. This finding is in line with previous research showing that under high threat or negative circumstances, people prefer larger interpersonal distances and show more reactance when this interpersonal distance decreases (Albert \& Dabbs, 1970; Greene, 1977; Okken, Van Rompay \& Pruyn, 2012; Schiffenbauer \& Schiavo, 1976). Overall these findings suggest that under high threat conditions, a larger interpersonal distance or a more spacious set-up makes respondents feel more at ease and more willing to cooperate, implying that they prefer to physically keep the threat at bay. 


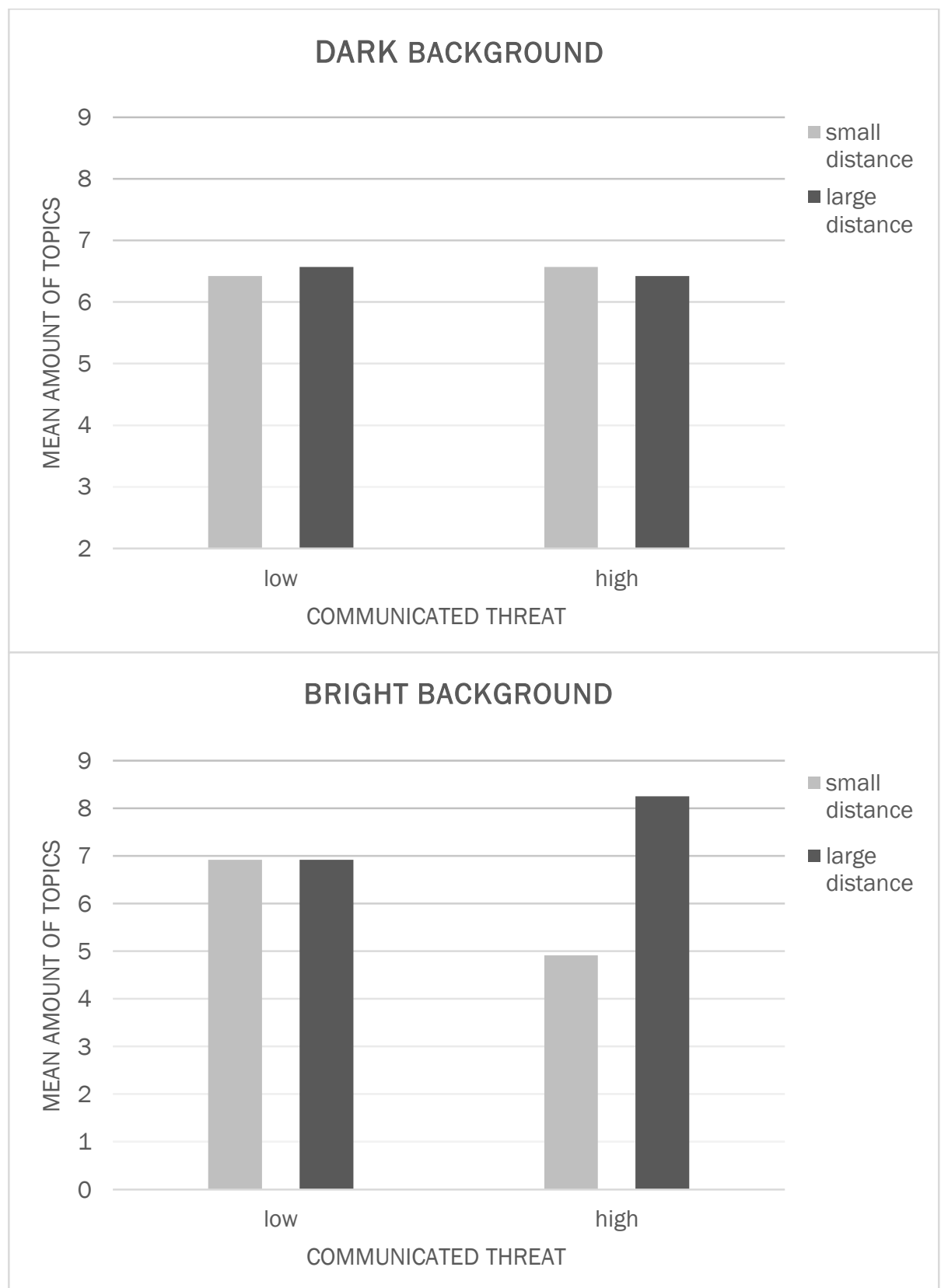

Figure 6.3. Three-way interaction between background brightness, communicated threat and interpersonal distance for the amount of topics, with the upper graph showing the bright background and the lower graph showing the dark background. 
Although similar patterns were found for the three-way effects of perceived spaciousness and self-disclosure, no mediating effect was found for perceived spaciousness. Therefore, the main hypothesis of the study could not be confirmed. Looking back on the items used to measure the spaciousness construct, all items reflect spaciousness perceptions as triggered by the physical environment. This could have steered the respondents' attention towards the physical environment surrounding them and away from the on-screen manipulations. Following this line of reasoning, these items may have been less appropriate as a measure for an online environmental manipulation. During online interaction, one is not physically surrounded by the online environment but merely looking at and interacting with a computer screen.

Arguably, in the online context experienced spaciousness can be conceptualized as the extent to which a user feels that the website offers room or space for selfexpression. With respect to website design, spaciousness perceptions may foremost relate to the actual distance between elements, and thus by the amount of white space in between, creating either the impression of a spacious and open, or alternatively, a closed and limiting design. Similar to how physical aspects influence self-disclosure, we expect that:

H4A: A spacious website design induces more perceived room for self-expression, thereby generating more self-disclosure than a constricting website design.

H4B: A spacious website design induces more perceived room for self-expression, thereby invoking less reactance than a constricting website design.

H4C: A spacious website design induces more perceived room for self-expression, thereby creating a more positive experience than a constricting website design.

At the same time however, the environment in which consumers access the internet may also impact consumer behavior. As mentioned earlier, research shows that physical environmental factors such as room lighting and color usage can influence spaciousness perceptions (cf. Flynn, Spencer, Martyniuk \& Hendrick, 1973; Kwallek, 1996; Manav, 2007; Oberfeld, Hecht \& Gamer, 2010). Therefore, in the second study, the effect of the physical surrounding is also examined. Based on the findings reported in the introduction and the results of study 6.1, we expect that room atmospherics impact self-disclosure via feelings of spaciousness. More specifically, when respondents are prompted to self-disclose in an online task, a brightly-lit room will induce feelings of spaciousness, thereby creating a more positive affective experience, invoking less reactance, and enhancing selfdisclosure. Conversely, a reduction of room brightness will increase feelings of confinement, in turn creating a less positive affective experience, thus invoking more reactance and a lowered willingness to self-disclosure. Hence: 
H5A: Brighter lighting conditions induce feelings of spaciousness, thereby generating more self-disclosure than dimmed lighting conditions.

H5B: Brighter lighting conditions induce feelings of spaciousness, thereby invoking less reactance than dimmed lighting conditions.

H5C: Brighter lighting conditions induce feelings of spaciousness, thereby creating a more positive experience than dimmed lighting conditions.

To test the hypotheses outlined, an online disclosure task was set up consisting of answering multiple personal open-ended and multiple choice questions to create an extensive personal profile for an alleged new social network that offers the possibility to meet new friends and potential partners. The computer tasks were arranged in two rooms that were identical apart from room brightness. This resulted in a 2 (room brightness: bright versus dimmed) by 2 (website design: spacious versus constricting) between subjects design.

\section{Study 6.2}

\section{Method}

\section{Participants}

A total of 92 participants (28 male, 64 female) were included in the study. Their mean age was 20.7 years $(S D=3.76)$. The study took place at a Dutch university. Participants could sign up for the study via an online system of the university. All participants were students enrolled in various (under)graduate programs at the university and received course credits for their participation.

\section{Procedure}

The participants were randomly assigned to one of the four conditions and were guided into one of the two experimental rooms. At the start of the experiment, participants were informed that the purpose of the study was to evaluate a new social network that offers you the option to create an extensive profile about yourself and get matched to possible friends and partners based on the information you provide. Next, some practical information was given about the use of the website and their tasks to test the website and evaluate it. At this point participants were instructed to give detailed answers to the questions and anonymity was guaranteed. After this, participants were invited to go to the website and create a profile by providing information and answer several questions concerning their personal appearance, their personality, their lifestyle and their dating preferences. These questions were used to measure self-disclosing behavior. Subsequently, participants were presented with a website-evaluation questionnaire comprising the 
other dependent measures. After completion of the experiment, participants were thanked for their participation and dismissed.

\section{Independent variables}

Two identical rooms were used on the campus of the university. In order to manipulate room brightness, covers that varied in transparency were placed over the ceiling lights, creating a bright and a dimmed room (bright room $M=179.6$ lux vs. dimmed room $M=20.2$ lux). A pilot study was conducted in which 26 participants were invited into one of the rooms and asked to evaluate the room on a seven-point Likert-type scale on several aspects, including room brightness. The results of the pretest confirmed that the covers had the desired effect on room brightness (bright room $M=4.46, S D=.77$ vs. dimmed room $M=3.92, S D=.76$; $t$ $=2.69 ; d f=24 ; p=.013)$.

To manipulate website design, two different screen designs were used (see Figures $6.4 \mathrm{~A}$ and $\mathrm{B}$ ). Following results on print advertisements showing that a more open or broad layout leads to a more positive evaluation (Olson, Pracejus \& O'Guinn. 2012; Pracejus, Olsen \& O'Guinn. 2006; Strong, 1926), the amount of white space was manipulated, thereby altering the broadness / narrowness of the screen-layout. For this purpose, the brightness (light grey vs. black), thickness (thin vs. thick) and size of the windows (large vs. small) were varied. The size of the answering field however remained constant across all designs so as to ensure that any effects would not be the resultant of having literally less space to provide an answer.

A pilot study was conducted in which 16 respondents were shown the series of 16 screen designs and asked to judge the website design in terms of spaciousness (i.e., broadness / narrowness), visual clarity and suitability for a website. All questions were measured on seven-point Likert-type rating scales. Based on the results of the pretest, two designs were selected that differed in terms of spaciousness $(M=5.13, S D=.81$ vs. $M=6.81, S D=.54 ; t=6.94 ; d f=30 ; p<$ $.001)$ but received similar scores for both visual clarity $(t<1, n s)$ and suitability for a website $(t=1.36 ; d f=30 ; p=.185)$.

\section{Questionnaire}

A seven-point Likert-type scale was used (1: "strongly disagree" to 7: "strongly agree") to measure the concepts of the study.

Experienced spaciousness. Experienced spaciousness was measured using the items: "I feel constricted inside this room" (reverse coded), "I feel confined inside this room" (reverse coded), "I have sufficient freedom of movement inside this room" and "I would easily feel suffocated inside this room" (reverse coded) ( $\alpha=$ $.87)$. 
Affective experience. To measure participants' affective experience, an affectmeasure was used comprising the items: "Inside this room I feel at ease", "Inside this room I feel unhappy" (reverse coded), "I feel uncomfortable inside this room" (reverse coded) and "This room gives me a pleasant feeling" ( $\alpha=.85$ ).

Room for self-expression. The experienced room for self-expression on the website was measured using the items: "I had sufficient space to show who I am", "I felt limited by the size of the field" (reverse coded) and "I had insufficient space to answer the question" (reverse coded) $(\alpha=.75)$.

Website aesthetics. Website aesthetics was measured using the items: "I find this website attractive", "This website has a nice design" and "I find this website uninteresting" (reverse coded) $(\alpha=.87)$.

Perceived ease of self-disclosure. Perceived ease of self-disclosure was measured with the items: "I felt inhibited from discussing personal information" (reverse coded), "I felt able to speak freely about myself", "I felt uncomfortable in sharing personal information" (reverse coded) and "It was hard for me to talk about myself" (reverse coded) $(\alpha=.89)$.

\section{Behavioral measures}

Self-disclosure measures. Self-disclosure was measured by analyzing the answers to the open ended questions. The topics of the questions were: own appearance, own personality, alcohol and drug usage, describing their ideal partner in terms of appearance and personality and their opinion about cheating while in a relationship. There were three measures for self-disclosure: word count and the amount of topics mentioned during the answer (Joinson, 2001; Omarzu, 2000). The third measure was derived from Joinson, Paine, Buchanan and Reips (2008) and consisted of a series of questions varying in sensitivity of which the amount of provided answers was counted. Participants could respond to each of the items either by providing an answer or by checking the box "I prefer not to say". The used items were: "Are you left or right handed", "Do you have a donor card", "Do you donate to charities", "Are you religious", "How many nights a week do you go out", "How many serious relationships did you have since the age of 18", "How long is your longest relationship to date", "How many different sexual partners have you had", "Of what gender were your sexual partners". 

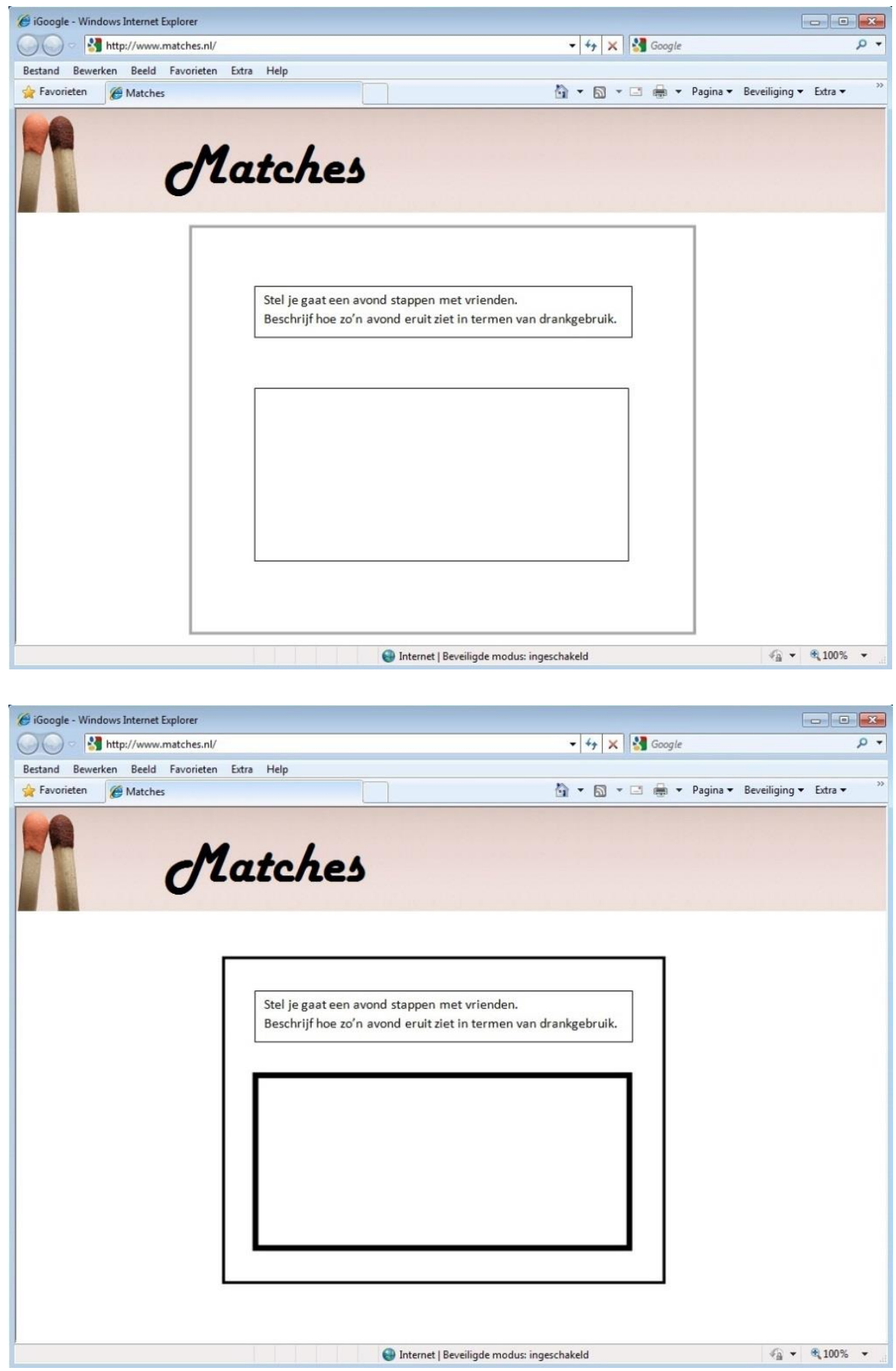

Figures $6.4 A$ and $B$. Pictures of the two used website designs. Website design was spacious for the upper picture and constricting for the lower picture. 
Reactance behavior. To measure reactance behavior, respondents were asked whether they were willing to participate in a follow-up study concerning the social network and whether they were willing to participate in future studies concerning online environments $(r=.52)$. Affirmative responses to these questions indicate less reactance.

\section{Results}

The results were analyzed for both gender and age using analysis of variance (ANOVA), but since none proved significant, there will be no further discussion of these variables ( $p>.10$ for all measures). A multivariate analysis of variance (MANOVA) was conducted, with room brightness and website design as independent variables, and with the self-disclosure measures, reactance behavior, experienced spaciousness, affective experience, room for self-expression, website aesthetics and perceived ease of self-disclosure as dependent variables. The analysis yielded a significant effect of room brightness $(F(10,79)=3.80, p<.000$, partial $\left.\eta^{2}=.33\right)$. Likewise, the multivariate effect of website design was significant $\left(F(10,79)=2.39, p=.016\right.$, partial $\left.\eta^{2}=.23\right)$. In addition, the multivariate effect of the interaction between room brightness and website design reached significance $\left(F(10,79)=2.76, p=.006\right.$, partial $\left.\eta^{2}=.26\right)$. Having established these effects, next the ANOVAs (comprising the same independent and dependent variables) are presented. An overview of all means and standard deviations of the found effects is given in Table 6.2.

\section{Experienced spaciousness}

Room brightness had an effect on experienced spaciousness $(F(1,90)=5.99, p=$ .016, partial $\eta^{2}=.14$ ) (See Table 6.2). As expected, experienced spaciousness scores for the bright room were higher than for the dimmed room. Additionally, website design also had an effect on experienced spaciousness $(F(1,90)=14.24$, $p<.000$, partial $\left.\eta^{2}=.06\right)$. The spacious website design yielded higher scores for experienced spaciousness compared to the constricting design. 


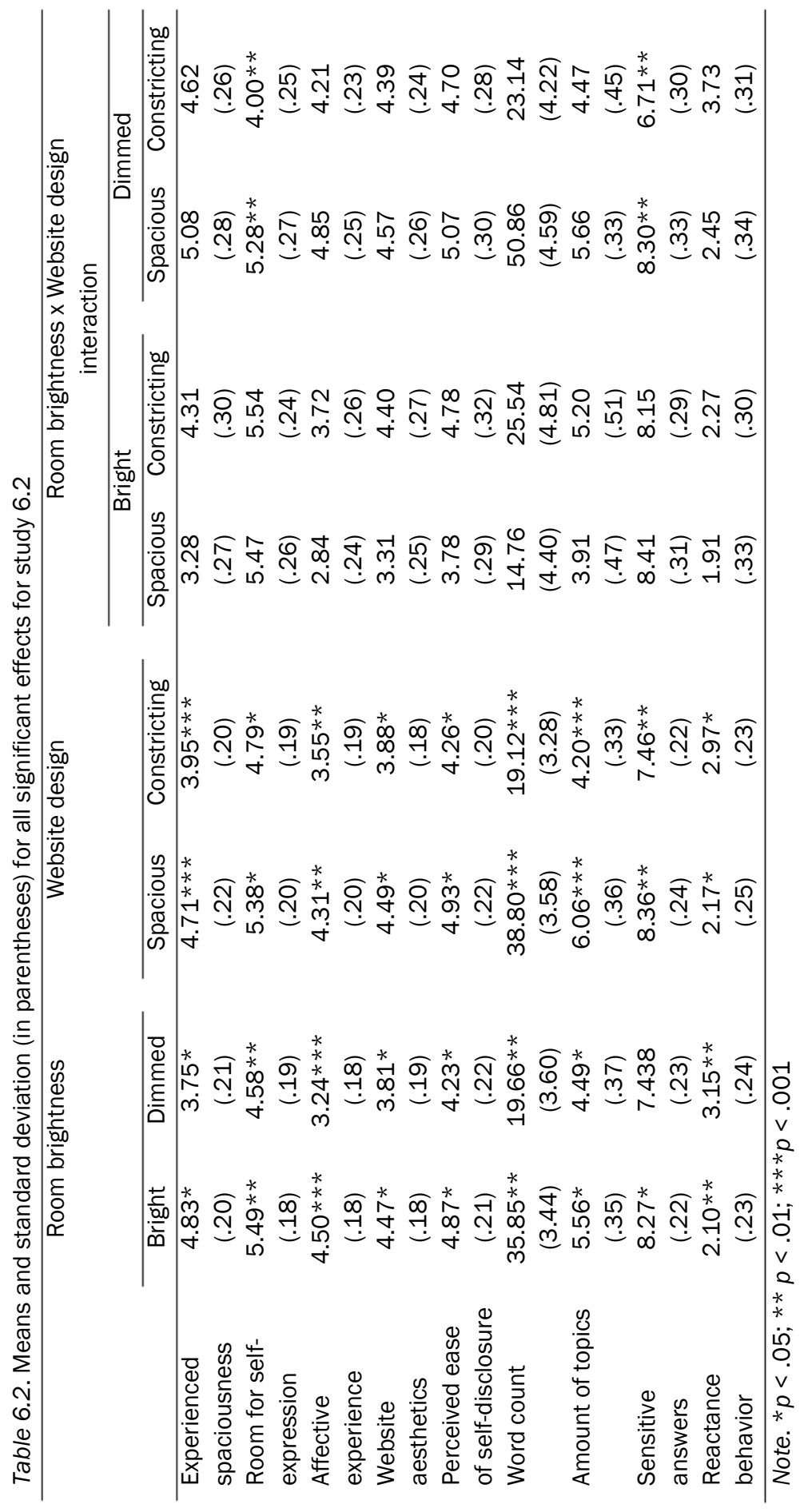




\section{Room for self-expression}

Again, room brightness had an effect on room for self-expression on the website ( $F$ $(1,90)=11.82, p=.001$, partial $\eta^{2}=.12$ ). Self-expression was easier in the bright room as compared to the dimmed room. In addition, website design had an effect on room for self-expression $\left(F(1,90)=4.662, p=.033\right.$, partial $\left.\eta^{2}=.05\right)$. Selfexpression was easier when the design was spacious than when the design was constricting. Also, an interaction was found between room brightness and website design $\left(F(1,88)=6.96, p=.010\right.$, partial $\eta^{2}=.07$ ) (see Figure 6.5). Further analysis of the simple main effects showed that in the dimmed room, there was a significant difference in room for self-expression for the different website designs ( $F$ $(1,88)=12.48, p=.001$, partial $\left.\eta^{2}=.12\right)$, indicating that self-expression was easier for the spacious than for the restricting design. In the bright room, this difference did not reach significance $(F<1, n s)$.

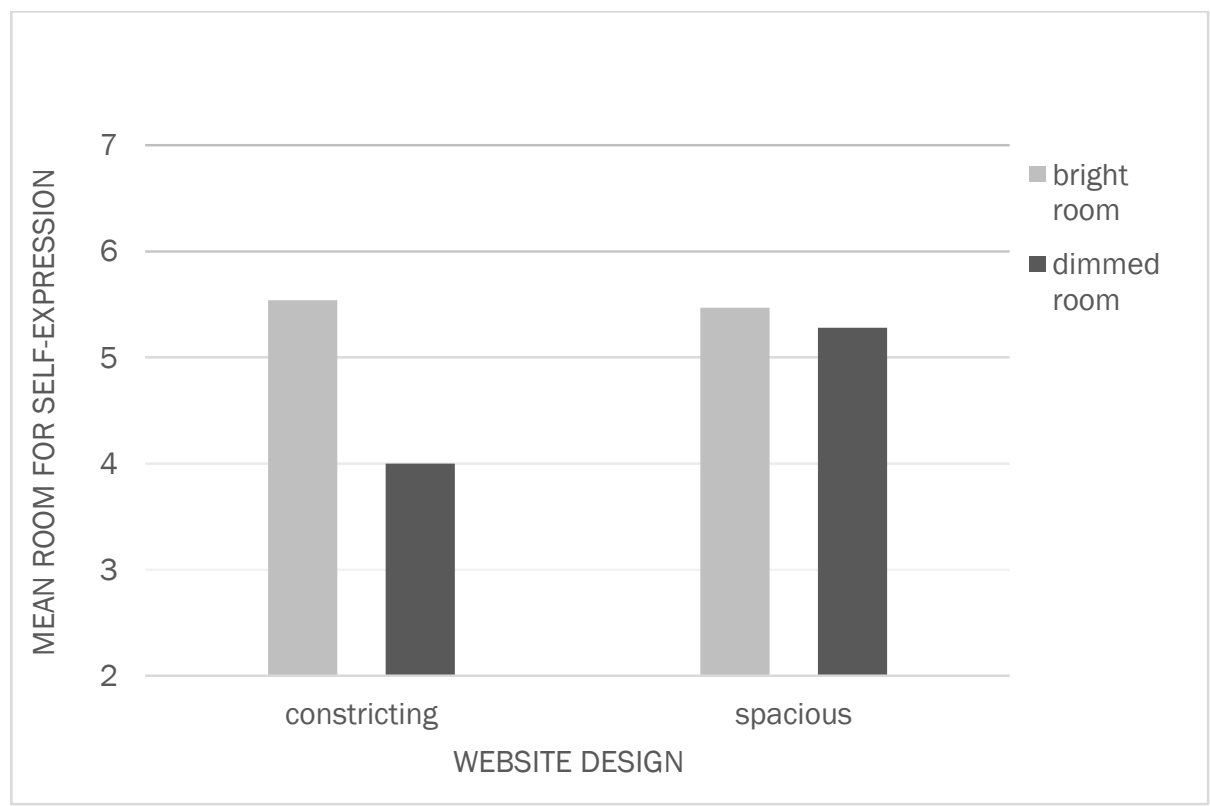

Figure 6.5. Interaction between room brightness and website design for room for selfexpression.

\section{Affective experience}

Similar to the results for perceived spaciousness and room for self-expression, room brightness yielded a significant effect on the affective experience $(F(1,90)=24.88$, $p<.000$, partial $\eta^{2}=.22$ ). The bright room triggered more positive affect than the dimmed room. In addition, website design had an effect on the affective experience $\left(F(1,90)=7.61, p=.007\right.$, partial $\left.\eta^{2}=.08\right)$. The spacious design triggered more positive affect than the constricting design. 


\section{Website aesthetics}

Room brightness had an effect on website aesthetics $(F(1,90)=6.34, p=.014$, partial $\left.\eta^{2}=.07\right)$. The bright room triggered more positive affect than the dimmed room. Furthermore, website design had an effect on website aesthetics $(F(1,90)=$ $5.27, p=.024$, partial $\left.\eta^{2}=.06\right)$. The spacious design triggered more positive affect than the constricting design.

\section{Perceived ease of self-disclosure}

Again, room brightness had an effect on perceived ease of self-disclosure $(F(1,90)$ $=4.48, p=.037$, partial $\left.\eta^{2}=.05\right)$. In the bright room the self-disclosure was perceived as easier than in the dimmed room. Website design also had an effect on perceived ease of self-disclosure $\left(F(1,90)=4.93, p=.029\right.$, partial $\left.\eta^{2}=.05\right)$. The spacious design triggered more ease of self-disclosure than the constricting design.

\section{Self-disclosure}

Word count. Room brightness had an effect on word count $(F(1,90)=10.57, p=$ .002 , partial $\eta^{2}=.11$ ). The amount of words used to answer the questions was higher in the bright room than in the dimmed room. In addition, the effect of website design on word count was significant $\left(F(1,90)=16.46, p<.000\right.$, partial $\left.\eta^{2}=.16\right)$. The amount of words used to answer the questions was higher when the website had a spacious design than when the website had a constricting design.

Amount of topics. The effect of room brightness on amount of topics reached significance $\left(F(1,90)=4.31, p=.041\right.$, partial $\left.\eta^{2}=.05\right)$. A larger number of topics was discussed in the bright room compared to the dimmed room. Furthermore, website design had an effect on the amount of topics $(F(1,90)=14.54, p<.000$, partial $\left.\eta^{2}=.14\right)$. The spacious design yielded a larger number of topics than the constricting design.

Sensitive answers. Room brightness had an effect on the number of sensitive answers that were provided $\left(F(1,90)=6.73, p=.011\right.$, partial $\left.\eta^{2}=.07\right)$. More sensitive answers were given in the bright room, compared to the dimmed room. Again, website design also had an effect on disclosure prompted by sensitive items $\left(F(1,90)=7.72, p=.007\right.$, partial $\left.\eta^{2}=.08\right)$. More sensitive answers were provided when the website had a spacious design than when the website had a constricting design. Additionally, an interaction was obtained between room brightness and website design $\left(F(1,88)=4.77, p=.032\right.$, partial $\left.\eta^{2}=.05\right)$ (see Figure 6.6). Further analysis of the simple main effects showed that in the dimmed room, there was a significant difference for the amount of sensitive answers $(F(1,88)=12.95, p=$ .001 , partial $\left.\eta^{2}=.13\right)$. In the bright room, no such effects were obtained $(F<1, n s)$. 


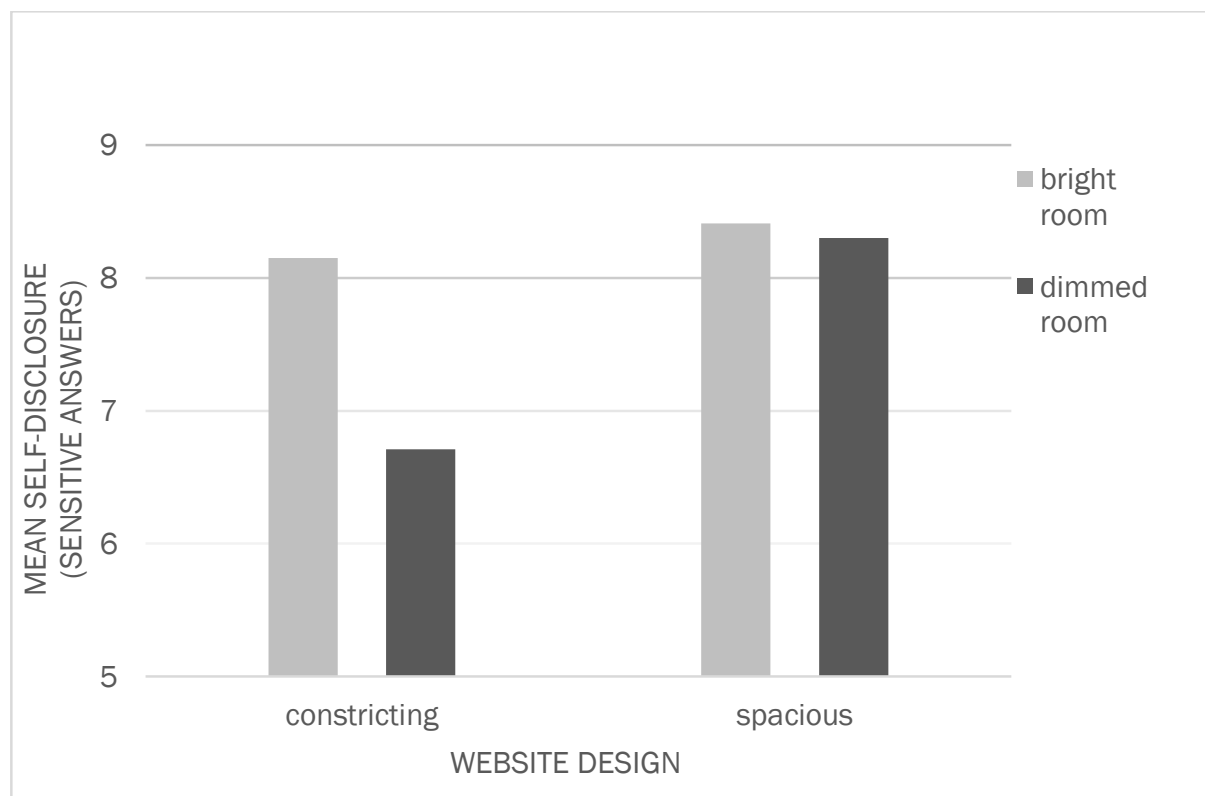

Figure 6.6. Interaction between room brightness and website design for sensitive answers.

\section{Reactance behavior}

The effect of room brightness on reactance behavior was significant $(F(1,90)=$ 9.94, $p=.002$, partial $\left.\eta^{2}=.10\right)$. The bright room yielded less reactance, as compared to the dimmed room. Also, website design also had an effect on reactance behavior $\left(F(1,90)=5.61, p=.020\right.$, partial $\left.\eta^{2}=.06\right)$. The spacious design yielded less reactance, as compared to the constricting design.

\section{Mediation analyses}

Mediation analyses, following the procedure outlined by Baron and Kenny (1986), were conducted in order to test whether the effects of room brightness and website design on the dependent variables (i.e., affective experience of the physical environment, affective experience of the online environment, perceived ease of selfdisclosure, the self-disclosure measures and reactance behavior) are indeed mediated by experienced spaciousness and by room for self-expression (see Figure 6.7). All reported regression coefficients are standardized. 


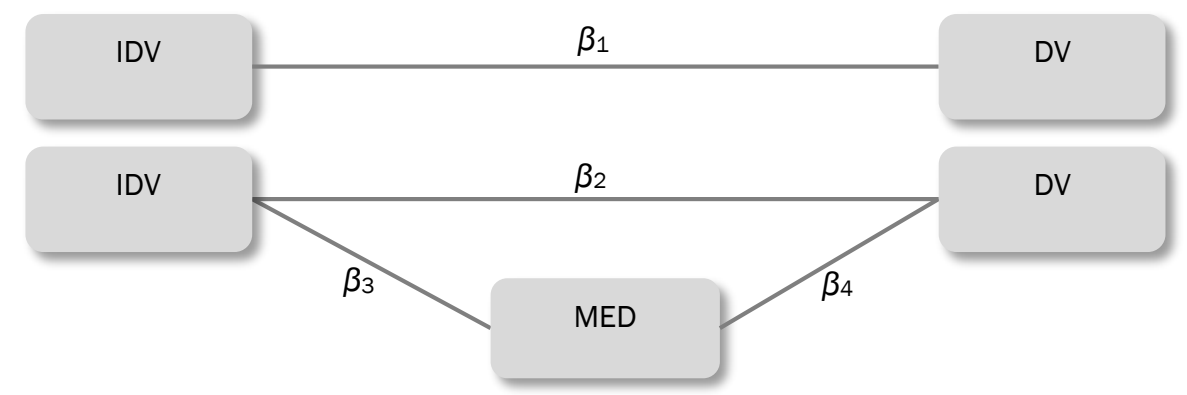

Figure 6.7. Mediation model, with room brightness or website design as the independent variable (IDV), perceived spaciousness or room for self-expression as the mediator (MED), and the self-disclosure measures or reactance behavior as dependent variable (DV).

First, it was tested whether room brightness influences the self-disclosure measure word count via experienced spaciousness. Analyses showed that the effect of room brightness on word count is significant ( $\beta=.32, p=.002)$. Likewise, the effect of room brightness on experienced spaciousness (the mediator) reached significance $(\beta=.37, p<.001)$, as did the effect of experienced spaciousness (the mediator) on word count (the dependent variable) $(\beta=.38, p<.001)$. When both room brightness and experienced spaciousness were included in the model, the effect of room brightness on word count became non-significant $(\beta=.15, p=.130)$, whereas the effect of experienced spaciousness on word count remained significant ( $\beta=.49$, $p<.001$ ). In addition, results of a Sobel test showed the indirect effect (of website design on word count via experienced spaciousness) to be significant (Sobel $z=$ $3.03, p<.01)$ confirming the mediating effect of experienced spaciousness on the relationship between room brightness and self-disclosure.

Similar mediation analyses were conducted for the other variables, and an overview of all significant mediations is given in Table 6.3. The overall pattern indicates that room brightness impacts the dependent measures via experienced spaciousness and that website design impacts the dependent measures via room for selfexpression. 


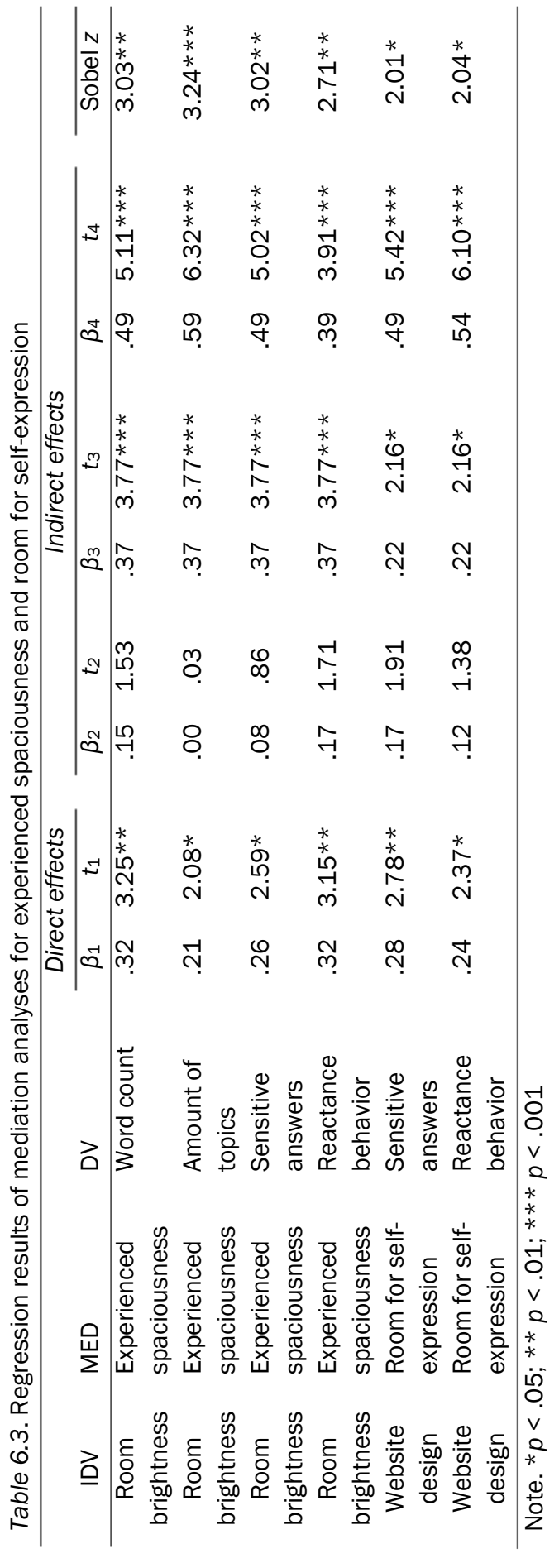




\section{Discussion}

The results confirm that experienced spaciousness is an underlying factor that can be used to explain effects of environmental manipulations on both affect and disclosing behavior. With respect to the physical environmental manipulation, results indicate that brightness can be used to manipulate feelings of spaciousness. As expected, increased spaciousness positively affected participants' judgments and their self-disclosure. In addition, experienced spaciousness negatively affected reactance, showing that participants are more willing to comply with requests in more spacious settings. With respect to website design, the results show that website atmospherics affecting spaciousness perceptions influence consumer behavior in a similar manner; participants experienced more room for self-expression in the spacious condition and likewise experienced more positive affect. In addition, increases of spaciousness negatively affected reactance levels, again showing that the willingness to comply is higher in more spacious settings.

Overall, the results show that self-disclosing behavior profits from a more spacious environment, which is in line with previous research claiming that people physically need space to disclose information about themselves (Okken, Van Rompay \& Pruyn, 2012). In addition, an interaction was found between the two manipulations, showing that poor physical conditions (i.e., low brightness) can be counteracted by a more spacious design. A possible explanation for this effect is that in a room with dimmed lighting a contrast effect occurs and a bright computer screen stands out more clearly and comes across as more spacious than it would in a brighter lit room. Interestingly, of the three self-disclosure measures that were used, the interaction only occurred for the sensitivity measure. This suggests that especially when it comes to discussing highly sensitive information people are sensitive to environmental cues and are more likely to notice, and be influenced by, website design.

\section{General discussion}

Importantly, the results indicate that effects of the manipulations are highly intertwined. That is, the results of the second study shows that the physical environmental manipulation also influenced the experienced room for selfexpression on the website and vice versa. In other words, the environment in which the task was completed influenced participants' judgments of the website. A possible explanation for these findings is that manipulating the physical environment not only influences how one feels within that environment but also affects perception. Arguably then, when judging to which extent a website meets the 
needs for self-expression, the current emotional state is used as a guideline as to how pleased one is with the website and steers behavior. This is corroborated by the fact that researchers studying the effects of website design often use models that stem from physical environmental psychology (e.g., Kaplan \& Kaplan, 1988; Mehrabian \& Russell, 1974) to explain the influence of online environments (Eroglu, Machleit \& Davis, 2001; Koo \& Ju, 2010; Richard, 2005; Rosen \& Purinton, 2004). Likewise testifying to such transference effects of environmentally induced perceptions to other stimuli, research shows that the physical environment is also consulted when judging a person's personality (e.g., judging personality based on bedroom or office appearance; Gosling, Ko, Mannarelli \& Morris, 2002) or when evaluating hospital services (Swan, Richardson \& Hutton, 2003) or travel agency services (Bitner, 1990). These results expose a source of concern for service providers since they can usually assert little or no control over the physical surroundings in which consumers consult and interact with websites.

However, the results reported also show that the online environmental manipulation affected the experienced spaciousness of the room. This shows that a brighter screen background or a more spacious website design can increase experienced spaciousness and can thus have a positive effect on both the affective experience and self-disclosure. The interaction between the physical and online environmental manipulation underscores this effect by demonstrating that in the second study the negative effect of a dimly lit room (i.e., low brightness) on self-disclosure can be attenuated by a more spacious website design. Hence, these combined findings suggest that service providers should ensure that website design connotes spaciousness perceptions as this may counteract potential negative effects caused by physical environmental factors restricting consumers' sense of space and freedom.

With respect to managerial implications, the findings calls for extra attention to factors impacting spaciousness perceptions or feelings of confinement during online interaction. Especially in healthcare contexts, high stress situations can easily appear when discussing threats to one's personal health. To prevent patients from verbally shutting down or perhaps even logging out of the conversation entirely, website design should induce feelings of freedom and spaciousness rather than restriction and confinement.

As for limitations, these studies were a first attempt to test the spaciousness hypothesis in an online environment, more research is needed to pinpoint which additional website design elements can impact spaciousness perceptions in order to provide website designers with guidelines as to how construe the online environment. As mentioned earlier, research on print advertising shows that the use of white space positively influences consumer evaluations and steers attention to 
the product and the brand name (Olson, Pracejus \& O'Guinn, 2012; Pracejus, Olsen \& O'Guinn, 2006; Strong, 1926), presumably because it triggers spaciousness perceptions. Future research should assess whether white space has similar effects when used in online environments and to what extent these effects are determined by spaciousness perceptions.

Of course, besides experienced spaciousness there are several other factors involved that may impact the willingness to self-disclose. For instance, during faceto-face conversations consumers may infer trustworthiness of service providers from their (nonverbal) behavior. During online interactions however, the other party is not visible and the discloser is often left uninformed about whom and how many others may read the disclosed information and for what purposes it will be used. Research shows that reassuring consumers by providing the privacy policy can increase their willingness to self-disclose (Andrade, Kaltcheva \& Weitz, 2002). In addition, a study interviewing over 2500 website users shows that website design was mentioned most frequently as a cue for evaluating the website's credibility (Fogg, Soohoo, Danielson, Marable, Stanford \& Tauber, 2003). Another method frequently used to gain trust of consumers is to implement social presence cues (e.g., pictures of employees) in the website design (Gefen and Straub 2004; De Vries \& Pruyn, 2007). Social presence refers to the degree to which one perceives the other party as "real" in mediated communication (Gunawardena \& Zittle, 1997). However, although positively affecting trust, it remains to be determined how the use of social presence cues such as pictures displaying faces affects consumers' willingness to self-disclose.

Finally, since website design has been shown to impact online behaviors such as purchase intentions and desire to stay on the website (Kim, Fiore \& Lee, 2007). Spaciousness might also be a key variable in accounting for these relationships. For instance, a website design that does not come across as spacious (i.e., a restricting design) may lead to a more negative browsing experience, prompting consumers to leave the site prematurely without checking out and completing pending transactions. Importantly, such unwarranted behaviors might spring forth from the same underlying issue (i.e., the experience of the website as too restricting). Findings from Study 6.1 showed that such avoidance behaviors may particularly surface when perceived threat is high. In the online context, high threats may not only stem from health concerns but may also reflect other types of risks such as financial risks, social risks, or risks related to privacy and anonymity of personal data submitted online. Hence, when consumers face such concerns, it might be particularly worthwhile to attend to website factors generating spaciousness perceptions. 
In sum, current research showed that spaciousness perceptions related to both the physical and the online environment impact (online) self-disclosure and related affective experiences; future research could explore the extent to which they also impact browsing behaviors and website experience in general. 
Chapter 7 begins with an overview of the main findings of the studies reported in the empirical chapters of this dissertation. Next, practical implications and contributions of the findings will be discussed, as well as limitations of the studies reported and possible directions for future research. 


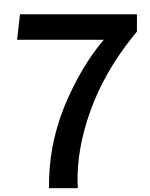

General Discussion 
"There is no such thing as empty space."

(René Descartes, Principles of Philosophy, 1644)

A growing body of research demonstrates the effects of several environmental factors on both affective experience and behavioral responses (Dijkstra, Pieterse \& Pruyn, 2006; Pressly \& Heesacker, 2001; Turley \& Milliman, 2000; Valdez \& Mehrabian, 1994). In addition, findings provide evidence for the importance of the environment for self-disclosure (Chaikin, Derlega \& Miller, 1976; Cohen \& Schwartz, 1997; Jourard \& Friedman, 1970; Lecomte, Bernstein \& Dumont, 1981; Sundstrom, 1975). As an explanation for such effects, it is argued that creating a comfortable or pleasant environment facilitates self-disclosure because such environments make people feel comfortable and at ease, which in turn induces selfdisclosure tendencies. Nevertheless, creating a pleasant environment can be considered challenging when taking into account that pleasure is determined by a large variety of factors inside a room. In addition, pleasure to a large extent depends on one's personal taste. Therefore, in this dissertation experienced spaciousness is introduced as another possible mediator in such relationships. Although research has demonstrated effects of spaciousness-related factors such as room size on self-disclosing behavior (Sundstrom, 1975), experienced spaciousness has not yet been examined as a possible mediator in such relationships. The present dissertation examined the effects of the physical environment on self-disclosing behavior and explored to what extent experienced spaciousness mediates this relationship. The previous chapters have examined the effects of different environmental factors, both in a physical and online environment.

\section{Main findings}

The research reported in this dissertation demonstrates and underlines the importance of environmental factors on our feelings and behavior. The studies all demonstrate that environmental factors of the four categories of the taxonomy presented in Chapter 2 are able to impact self-disclosure. In addition, the results underline the importance of experienced spaciousness and revealed its mediating effect on the relationship between environmental factors and self-disclosing behavior, thereby contributing to our knowledge in both the fields of environmental psychology and communication research. 
In line with previous research on self-disclosure, it is confirmed that self-disclosure is affected by the physical (and online) environment. Also, acknowledging the influence of experienced spaciousness increases knowledge of the underlying processes involved. Much previous research on environmental factors influencing self-disclosure has focused on factors related to the conversational partner, such as interpersonal distance and social density, and far less attention has been paid to the effects of the lifeless environmental factors, such as spatial density and interior design (for an overview, see: Cozby, 1973; Ignatius \& Kokkonen, 2007; Omarzu, 2000). With respect to experienced spaciousness, previous research has thus merely revealed the tip of the iceberg and leaves many variables to explore, both in the physical and in the online world. The studies presented in this dissertation provide a good basis for future research to further increase understanding of experienced spaciousness effects on communicative behavior.

By examining the full breadth of the used taxonomy, many possibilities have been created for other environmental research on spaciousness perceptions; there are still many factors of which it is yet to be determined to what extent they impact affective and behavioral responses. Furthermore, experienced spaciousness may also be of use in explaining seemingly contradicting findings of previous research. For instance, both Gifford (1988) and Miwa and Hanyu (2006) investigated the effects of lighting conditions on self-disclosure. Gifford (1988) showed that bright lighting stimulated self-disclosure of students during a plenary discussion. Miwa \& Hanyu (2006), however, showed that dim lighting enhanced self-disclosure of students in a counseling setting. Since neither experiments incorporated spaciousness perceptions in their design, it is interesting to determine to what extent this mediator can account for the effects observed.

The finding that insufficient spaciousness heightens reactance provides more insight in the bigger picture of the processes at hand. In this dissertation the focus lies on self-disclosing behavior, but the findings for reactance (Chapter 6) suggest that beside the effects self-disclosure, other behavioral responses may also be affected by spaciousness manipulations. As discussed in Chapter 2, reactance has been found to transpire in other ways (i.e., adopting a more varied product choice; Levav \& Zhu, 2009), therefore it seems possible that it may also impact other behaviors besides self-disclosure.

The results presented can be of use to architects and interior designers of service environments, but also to service providers, who may rearrange or add furniture or decorations without any consideration of the possible effects of such changes. The studies demonstrate that not only large radical changes to environments but also small subtle changes of factors such as brightness can have an effect. In addition, the findings regarding experienced spaciousness provide more insight in the 
underlying process and thereby may present a more useful guideline for designing or reconstructing environments than the isolated effects of different environmental factors. Further investigation of other factors belonging to the four categories of the taxonomy (see Figure 2.1) may present architects and designers with even more opportunities to incorporate spaciousness in their designs. In addition, it can increase knowledge of service providers about the effects their service environment, including themselves, have on their client and allows them to put this knowledge to good use. Service provider should therefore keep in mind that investments to improve their service environment will not merely impact client selfdisclosure but may improve the whole process of service provision and also increase their clientele.

It is demonstrated that spaciousness perceptions are not only affected by manipulations of the physical environment but that they are also sensitive to the level of threat that is present during communication. The results show that environments come across as less spacious during high threat conversations as opposed to low threat conversations. This presents a word of caution to service providers; despite improving the physical environment, clients can still feel cornered by a service provider's verbal behavior.

In addition, besides verbal responses in terms of self-disclosure, the results presented show that non-verbal behavior is also affected by spaciousness perceptions. The results demonstrate that as a result of the environmental manipulations respondents' bodily posture, amount of eye contact and amount of leaning on the table were affected during seated dyadic conversation discussing different intimate topics. Overall the results show that too confining settings encourage respondents to increase distance by adopting a less open bodily posture, leaning further backwards and making less eye contact.

\section{Self-disclosure and beyond}

In this dissertation, influences of environmental factors are mainly explored in physical and mental health care settings. Conversations between patient and health care provider usually form the basis of the consult, which makes the health care provider dependent on the information provided and self-disclosure an important factor in such contexts. However, as mentioned in Chapter 2, there are many other service environments in which the service provider requires information from their clients to provide the right service and therefore client self-disclosure is necessary to understand their service request. For example, in Chapter 6 a different context is introduced in which self-disclosure is also expected; creating a profile for an online 
dating website. Here, the information provided is not necessarily related to one's physical or mental health, but it is still considered personal.

The used context in Chapter 6 also presents another form of communication that involves self-disclosure; online communication. New websites emerge on a daily basis (150.000 new URLs each day; DailyChanges.com) and a growing number of businesses is shifting their attention to the internet. With this, the amount of online self-disclosure is also increasing. Think of activities such as filling out profiles, sharing information on social network sites, writing blogs, posting pictures, writing product reviews or chatting with family members, friends or strangers. Although the information is not disclosed verbally or during a face-to-face conversation, it is still considered self-disclosure since it considers personal information that is provided with the notion that others will read this information (Barak \& Gluck-Ofri, 2007; Gifford, 1988; Ignatius \& Kokkonen, 2007; Joinson, 2001; Joinson, Paine, Buchanan \& Reips, 2008; Jourard \& Friedman, 1970). This broad variety of other (service) settings in which self-disclosure plays a role presents us with many environments that can potentially benefit from the findings in this dissertation by improving client self-disclosure.

The results of Chapter 4 show that nonverbal behavior can be used as a means to adjust an uncomfortable setting by increasing spaciousness, which stresses the need for service providers to become more aware of the seemingly innocent movements of their client and respond to such actions by respecting their need for a more spacious set-up and not to keep closing the distance that the client wants to retain. This introduces a different perspective on aggressive or assertive sales techniques, especially with respect to long term commitment of clients. The results demonstrate that experienced spaciousness is not only affected by the physical environment but also by nonverbal (and verbal) behavior of the conversational partner. Therefore, a client may also feel physically cornered by certain nonverbal behaviors displayed by the service provider (e.g., too much eye contact, leaning forward, small interpersonal distance). In turn, this gives the client a confining feeling that may heighten reactance and thus lower the willingness to self-disclose or commit. Arguably, some nonverbal adjustments are not an attempt to optimize poor spaciousness conditions. For instance, leaning backward can signal a need for more spaciousness but can also be the result of relaxation. Therefore, future research should not only focus on nonverbal behavior, but should also include physiological measurements such as muscular tension, blood pressure, heart rate, respiratory rate or other measurements related to arousal (Sapolsky, 1998) to determine the level of anxiety that respondents are experiencing during such circumstances. 


\section{Spaciousness}

Overall, the results suggest that in an effort to improve self-disclosure during conversation, one should not only figuratively provide space by having an open and understanding attitude, creating silences to let the other person speak and finish their story but also literally provide space by creating a more spacious set-up with the use of the physical environmental factors. In this dissertation it is demonstrated that spaciousness can be influenced by different environmental factors belonging to each of the categories of the taxonomy discussed in Chapter 2 (see Figure 2.1). It seems worth exploring effects of other factors of each category, which hopefully results in more possibilities for manipulating experienced spaciousness. Of course, different environments may call for different measures to be taken. For instance, a minimalistic interior design may create a more spacious set-up in a therapist's office but perhaps this also makes the therapist come across as cold or distant and, as a consequence, will make the interaction less pleasant or less intimate and perhaps will also hinder self-disclosure. Also, spaciousness can be increased by making the boundaries of an environment more transparent. However, placing large windows and thereby increasing visibility of the outside world also makes people more conscious of being seen by outsiders looking in. One can imagine that during an intimate dinner in a restaurant or a private conversation in a doctor's office, feelings of being put on display can negatively impact the atmosphere. It thus seems important to determine which factors should be altered to improve spaciousness perceptions without bringing about an opposite effect by creating an uncomfortable atmosphere or unrealistic setting. In addition, choosing a more overall approach and altering several factors at once may result in creating an unrealistic environment, which can lead to negative outcomes. For example, research on prototypicality shows that a physician displayed in a less prototypical office is judged less favorable (Swan, Richardson \& Hutton, 2003; Ward, Bitner \& Barnes, 1992). In turn, such negative effects can perhaps also impact communicative behavior such as self-disclosure. Therefore, one should refrain from adopting manipulations that were successful in another service environment but first consider the implications of such generalizations.

When taking into account the finding of study 6.2 that a spacious setting yields less reactance than a confining setting, one can imagine that in service settings a more spacious setup may not only yield more self-disclosure but, as a result of lowered reactance, this may also transpire in other behavioral reactions. For instance, a spacious setting in a restaurant may result in more consumption by taking your time and ordering more courses, whereas a confining setting in the same restaurant may result in a different set of choices; you may want to spend less time and order fewer courses. Furthermore, as a result of heightened reactance, you may be uneager to

order recommended dishes such as de daily special or the chef's special. In 
addition, the findings call to question what environments should be used when interrogating suspects. A too confining environment when using intimidating techniques, such as applying pressure or imposing restrictions, may heighten reactance and reduce self-disclosure. This underlines that caution should be taken in generalizing different types of environments with respect to the effects of environmental manipulations on experienced spaciousness and self-disclosing behavior.

\section{Origins of the need for spaciousness}

The question however remains why spaciousness is important to us. According to Stamps (2005) the basis for our spaciousness needs can be found in the prospect refuge theory (Appleton, 1996). This theory states that our preference for certain environments is evolutionary determined. Prospect refers to the ability to see the environment and possible threats and refuge refers to the possibility to hide in the environment. According to the theory we prefer environments in which both prospect and refuge are present because they increase our survival chance. In addition, Hediger's (1955) concept of flight distance notes that, besides sight, movement is also of importance. When faced with dangerous situations, the ability to move through an environment to reach safety is important to survival. These two theories thus state that our preference for more spacious surroundings stems from our ancestors and their survival techniques.

These theories are supported by a large scale poll conducted for the artists Vitaly Komar and Alexander Melamid by public opinion firms in ten different countries (Dutton, 2009; Komar, Wypijewski \& Melamid, 1997). With this poll, participants' artistic preferences were determined, including their favorite works of art, pictures, sceneries, animals, colors and so forth. Based on the results of the poll, the artists were able to paint a most-wanted and a least-wanted painting for every country that was included in the poll. One result of this poll that is of particular interest to the current context is that there was much agreement across the different countries with respect to the most-wanted paintings. Participants from the different countries all preferred similar graphic representations, which included a landscape with trees and open areas. Although the study was set up to investigate peoples preferences towards art, it is an interesting finding that participants from countries all over the globe (e.g., China, Kenya, Iceland, America) gravitated towards the same sceneries, suggesting an evolutionary basis for scenic preferences.

Furthermore, the significance of space also follows from neurophysiology research of Epstein and Kanwisher (1998), who demonstrated that a particular area in the 
human parahippocampal cortex named the parahippocampal place area (PPA) reacts to information related to the local visual environment. Their results show that the PPA responds more strongly to scenes with information about the spatial layout than to scenes without information about the spatial layout. More specifically, the PPA responds significantly stronger to scenes with spatial layout information than to scenes without spatial layout information; both empty rooms and furnished rooms yield a strong response of the PPA, whereas the same furniture placed against a straight background (no spatial layout information) yields a significantly weaker response.

\section{Cultural influences on spaciousness needs}

Besides evolution, another important determinant of spaciousness preferences is one's cultural background. Although little to no research can be found that examines cultural differences in spaciousness perceptions, research focusing on personal space provides evidence for a cultural influence on experienced spaciousness (Aiello, 1987; Altman, 1975; Hall, 1965; Hall 1966; Høgh-Olesen, 2008; Remland, Jones \& Brinkman, 1995). For instance, Hall (1966) distinguishes non-contact (e.g., Northern European, Northern American) and contact (e.g., Mediterranean, Arabic, Hispanic) cultures. Individuals from non-contact cultures maintain larger interaction distances and are less likely to live in close physical contact with others than individuals from contact cultures. Also, individuals from contact cultures would be more tolerant to crowded situation than individuals from non-contact cultures. This distinction is supported by findings of, amongst others, Aiello (1987), Remland, Jones and Brinkman (1995) and Høgh-Olesen (2008). For instance, Høgh-Olesen examined personal space preferences in several countries and found that participants from Greenland, Finland and Denmark systematically maintained a larger interpersonal distance than participants from Italy, India and Cameroon. These results confirm Hall's classification of Northern European countries as non-contact countries and Mediterranean countries as contact countries.

It can thus be expected that the same interpersonal distance or perhaps the same room can come across as normal for an individual from a contact culture while it comes across as too intimate or confining to an individual from a non-contact culture. In addition, According to Altman (1975), there is an intercultural difference in living situations and some cultures are more used to crowded living than others. For instance, squatter families in slum areas are used to living in crowded small shacks with one room, while Northern Americans have big houses consisting of multiple rooms. Altman states that our perception of environments in terms of 
spaciousness to a large extent depends on our cultural background. In addition, this underlines the importance of earlier experiences in spaciousness perceptions.

\section{Embodiment and spaciousness perceptions}

The importance of experiences also follows from research on embodied social cognition or embodiment (Lakoff \& Johnson, 1980). According to Lakoff and Johnson (1980) the human conceptual system uses only a small set of concepts which stem directly from our experience and are defined in their own terms. These fundamental concepts include basic spatial relations (up versus down, front versus back), physical ontological concepts (e.g., containers, entities) and actions (e.g., moving, eating). All other concepts that we encounter, that do not directly stem from our physical experience are metaphoric in nature. To structure and understand such metaphoric concepts, we use metaphoric mapping of the small set of experiential concepts (Lakoff \& Johnson, 1980). In other words, our earlier physical experiences influence our rationale and to comprehend the situations we encounter, we use information from our physical experiences. This connection between physical experiences and abstract concepts influences, amongst others, our affective and behavioral responses towards others. For instance, Williams and Bargh (2008) investigated the effect of physical warmth on social judgments. Their findings revealed that experiencing physical warmth by holding a warm beverage increases feelings of interpersonal warmth, whereas holding a cold beverage increases feelings of interpersonal coldness. Also, Boroditsky (2000) examined whether abstract thought is structured through metaphorical mappings from the more concrete domain of space. Her results show that activation of concepts such as "forward" or "up" influences judgments of temporal relations. Furthermore, Meier and Robinson (2004) investigated the effect of spatial location and the affective valence of words. Their results show that evaluations of positive words were faster when these words were presented in the upper half of the computer screen, whereas evaluations of negative words were faster when presented in the lower half of the screen.

Furthermore and perhaps more in line with the context of this dissertation, Williams and Bargh (2008) studied the effects of spatial distance on emotional distance and showed that activating distance makes respondents experience weaker attachments to their relatives, whereas activating closeness makes them experience stronger attachments. In other words, a manipulation of experienced physical distance influenced feelings of psychological distance. A comparable effect was found by Meyers-Levy and Zhu (2007), who manipulated ceiling height (i.e., physical space) and found an effect on creativity and feelings of freedom (i.e., 
psychological space). Furthermore, Levav and Zhu (2009) manipulated physical space in supermarkets and also found an effect on psychological space.

These findings all demonstrate the strong connection between physical experiences and mental concepts. This connection also surfaced in the studies presented in this dissertation. The findings show that manipulating the experienced physical space, either by manipulating the actual amount of space (e.g., room size, interpersonal distance) or by creating an illusion of more or less space (e.g., room brightness, website design), impacts ones experienced psychological space or freedom. Each of the empirical chapters provides evidence that limiting the experienced physical space lowers the willingness to self-disclose and creates a more negative experience. A similar effect was found by Levav and Zhu (2009), who found that narrower aisles increased feelings of confinement, which was counteracted by respondents by making more varied products choices. Levav and Zhu (2009) suggest that restrictions of the physical space invoked feelings of reactance, which emerges as a refusal to cooperate or comply with behavioral norms or to perform expected behavior, in their study emerging as a more varied product choice. In the current research, reactance emerged as a lowered willingness to self-disclose. This line of reasoning was confirmed in Study 6.2 of Chapter 6, where it is demonstrated that limiting the experienced spaciousness indeed heightens reactance behavior.

While the studies presented focus mainly on self-disclosure, it is likely to expect that other communicative behaviors will be influenced alike by manipulating experienced spaciousness and, with that, elevating reactance. Besides selfdisclosure, it is also desirable for service providers that their clients listen to their conversational partner, dare to ask questions, or to overall participate more actively during the conversation. For instance, Chapter 4 demonstrates that besides selfdisclosure, non-verbal behavior is also affected by the environmental manipulations; effects were found for bodily posture and eye contact. These results, together with the findings reported in Chapter 6 for reactance, which show that lacking spaciousness settings heightens reactance and thus lowers the willingness to comply, suggest that it isn't merely self-disclosure that is affected by a less spacious set-up but that this experienced limitation has a more comprehensive effect on how one feels and behaves. Overall, these findings provide evidence that not only self-disclosure but communicative behavior as a whole can benefit from improving environmental circumstances and thereby increasing experienced spaciousness. One can imagine that a client experiencing heightened reactance during interaction with the service provider not refuses to self-disclose but perhaps also has reservations about making a commitment, signing a contract or spending money. 
Arguably, heightened reactance caused by unmet spaciousness needs may not only lower the participation in interactions but perhaps also leads people to overall be less accepting of others. In turn, this may decrease the willingness to make physical contact and intensify aggressive behavior towards others or even invoke intolerance or discrimination of minorities (Bell, Green, Fisher \& Baum, 2001). Research on density and crowding indeed shows that crowded situations can decrease prosocial behavior (Bickman, Teger, Gabiele, McLaughin, Berger \& Sunaday, 1973) and can increase physiological arousal (Evans, 1979; Evans, Lepore, Shejwal \& Palsane, 1998), and aggression (Baum \& Koman, 1976; Palmstierna, Huitfeldt \& Wistedt, 1991; Pontell \& Welsh, 1994). For instance, research of Evans (1979) investigated crowding effects of students during study sessions in either a large or small room. The results showed that participants in the high-density situation had higher heart rates and elevated blood pressure than participants in the low-density situation. In addition, Pontell \& Welsh (1994) studied the effect of density on aggression in prison records and found high correlations between population density and aggressive behaviors. Future research should examine whether experienced spaciousness can account for the found effects in density and crowding research and whether environmental manipulations can help increase spaciousness perceptions and with that reduce the negative effects of high dense circumstances.

\section{Limitations and directions for future research}

The goal of this dissertation was to create more insight in the impact of environmental factors on self-disclosing behavior. As mentioned in Chapter 2, environments are very complex and contain many stimuli that can possibly affect our cognition and behavior. The taxonomy in Figure 2.1 shows a categorization of these factors into four different groups. In this dissertation, factors were tested from all four categories. However, there are still many other factors for which it is yet to be determined to what extent they impact spaciousness perceptions and, in turn, behavioral responses. With the complexity of the environments that surround us comes the need for good research to examine such environmental effects. The studies presented in this dissertation use a variety of methods to investigate the environmental effects on behavioral intentions and behavior to ensure external validity. However, although the effects on both self-disclosure intentions and actual self-disclosing behavior have been found in such controlled environments, it remains to be tested to what extent the effects can be replicated in field experiments. Also, each of the methodological chapters presents interaction effects found between the environmental manipulations, therefore it can be expected that in field settings (i.e., complex environment with multiple environmental factors) 
even more interaction will take place, which further stresses the need for field experiments.

In addition, the focus of this dissertation is on the mediating role of spaciousness perceptions. However, this does not rule out the fact that there are more mediators yet to be determined that also affect self-disclosure tendencies. For instance, Chapter 5 shows an effect of a brightness manipulation on the respondents' judgments of the physician in terms of liking. However, this effect was not mediated by experienced spaciousness but by positive affect. This shows that besides spaciousness perceptions, other aspects such as positive affect but perhaps also trust or privacy perceptions can be of influence. For instance, one can imagine that indiscrete handling of client dossiers, by leaving them out in the open on a desk, negatively impacts feelings of trust and in turn decreases the willingness to disclose sensitive personal information.

Chapter 3 demonstrates that personality traits of the discloser strongly affect the impact of environmental factors; interaction effects were found between the environmental manipulations and the personality trait reservedness. However, this is yet one of many possible personality traits that can impact how one reacts in social interactions, or perhaps influence how one responds to a certain amount of spaciousness (Brody \& Ehrlichman, 1998, Dijkstra, Pieterse \& Pruyn, 2008). For instance, the trait of extraversion / introversion has been linked to a person's openness or secretiveness (Brody \& Ehrlichman, 1998). It can be expected that such traits, that influence the ease of social interaction, also impacts someone's willingness to self-disclose. In addition, personal differences in sensitivity to environmental factors may impact the effect of such factors on self-disclosing behavior. For instance, stimulus screening ability has been found to affect the sensitivity towards colors. It can be expected that environmental manipulations have a more pronounced effect on high-screeners, who are more sensitive to environmental factors, than on low-screeners, who are less sensitive to environmental factors.

Of course, the consumer is not the only one who is affected by the environmental factors. Since, at least during face-to-face interaction but also during online interactions when both parties use the same screen design, the service provider is present in the same environment, both can be affected by the environment. In addition, as mentioned in Chapter 2, self-disclosing behavior is also affected by characteristics of the conversational partner, including their behavior (Morton, 1978; Nilsson, Strassberg \& Bannon, 1979; Taylor \& Gill, 1983). Thus, besides a direct effect of environmental factors on consumers, there can also be an indirect effect via the service provider's behavior. Furthermore, the research reported in this dissertation focusses on interactions that take place during first conversations with 
service providers, because during such first meetings, or intakes, consumers are expected to disclose most information about themselves and to communicate their service request. It remains to be determined whether and how spaciousness needs change over time when several interactions have taken place between client and service provider and some level of bonding has already occurred. Based on research of Hall (1965) it can be expected that over time, when two conversational partners are more familiar with each other, spaciousness needs decrease and less spacious set-ups will suffice or even be preferred. However, research is needed to determine whether spaciousness needs indeed change over time and whether this is the case for all service environments. It is possible that over time a relationship between a physician and patient can become more personal and spaciousness needs will decrease, while a relationship between a banker and his client remains more business-like and in this case spaciousness needs will perhaps not decrease.

Taken together, the current research contributes to the still growing body of evidence of environmental influences of our affect, cognition and behavior. The experiments and simulation studies demonstrate that the environment is not merely a bystander during conversations but influences how we communicate. In addition, current research presents a new way of looking at the environment. It introduces experienced spaciousness as a mediating factor in the relationship between the environment and our behavioral responses. This provides us with new insights in explaining environmental influences and can hopefully help to further improve our built environment. 
References 
Acking, C. A., \& Küller, R. (1972). The perception of an interior as a function of its colour. Ergonomics, 15(6), 645-654.

Aiello, J. R. (1987). Human spatial behavior. In D. Stokols \& I. Altman (Eds.), Handbook of Environmental Psychology (Vol. 1, pp. 505-531). New York: Wiley.

Akalin-Baskaya, A., \& Yildirim, K. (2007). Design of circulation axes in densely used polyclinic waiting halls. Building and Environment, 42(4), 1743-1751.

Albas, C. (1991). Proxemic behavior: A study of extrusion. Journal of Social Psychology, 131(5), 697-702.

Albas, D. C., \& Albas, C. A. (1989). Meaning in context: The impact of eye contact and perception of threat on proximity. Journal of Social Psychology, 129(4), 525-531.

Albert, S., \& Dabbs, J. M. (1970). Physical distance and persuasion. Journal of Personality and Social Psychology, 15(3), 265-270.

Altman, I. (1975). The environment and social behavior. Monterey, CA: Wadsworth Publishing.

Altman, I., \& Taylor, D. (1973). Social penetration: The development of interpersonal relationships. New York: Holt, Rinehart and Winston.

Andrade, E. B., Kaltcheva, V., \& Weitz, B. (2002). Self-disclosure on the web: The impact of privacy policy, reward, and company reputation. Advances in Consumer Research, 29(1), 350-353.

Argyle, M., \& Dean, J. (1965). Eye-contact, distance and affiliation. Sociometry, 28(3), 289-304.

Appleton, J. (1996). The experience of landscape. New York: John Wiley.

Baird, J. C., Cassidy, B., \& Kurr, J. (1978). Room preference as a function of architectural features and user activities. Journal of Applied Psychology, 63(6), 719-727.

Baker, J. (1986). The role of the environment in marketing services: the consumer perspective. In J. A. Czepiel, C. A. Congram \& J. Shanahan (Eds.), The services challenge: integrating for competitive advantage (pp. 79-85). Chicago: American Marketing Association.

Barak, A., \& Gluck-Ofri, O. (2007). Degree and reciprocity of self-disclosure in online forums. CyberPsychology and Behavior, 19(3), 407-417.

Baron, B. M., \& Kenny, D. A. (1986). The moderator-mediator variable distinction in social psychological research: Conceptual, strategic and statistical considerations. Journal of Personality and Social Psychology, 51(3), 11731182.

Baron, R. A., Rea, M. S., \& Daniels, S. G. (1992). Effects of indoor lighting (illuminance and spectral distribution) on the performance of cognitive tasks and interpersonal behaviors: The potential mediating role of positive affect. Motivation and Emotion, 16(1), 1-33. 
Bateson, J. E. G., \& Hui, M. K. (1992). The ecological validity of photographic slides and videotapes in simulating the service setting. Journal of Consumer Research, 19(2), 271-281.

Baum, A., Fisher, J. D., \& Solomon, S. K. (1981). Type of information, familiarity and the reduction of crowding stress. Journal of Personality and Social Psychology, 40(1), 11-23.

Baum, A., \& Koman, S. (1976). Differential response to anticipated crowding: Psychological effects of social and spatial density. Journal of Personality and Social Psychology, 34, 526-536.

Bell, P. A., Greene, T. C., Fisher, J. D., \& Baum, A. (2001). Environmental psychology ( $5^{\text {th }}$ edition). Fort Worth: Harcourt College Publishers.

Bickman, L., Teger, A., Gabiele, T., McLaughin, C., Berger, M., \& Sunaday, E. (1973). Dormitory density and helping behavior. Environment and Behavior, 5, 465490.

Bitner, M. J. (1990). Evaluating service encounters: The effects of physical surroundings and employee responses. Journal of Marketing, 54(2), 69-82.

Bitner, M. J. (1992). Servicescapes: The impact of physical surroundings on customers and employees. Journal of Marketing, 56(2), 57-71.

Boroditsky, L. (2000). Metaphoric structuring. Understanding time through spatial metaphors. Cognition, 75(1), 1-28.

Brody, N., \& Ehrlichman, H. (1998). Personality psychology. The science of individuality. Upper Saddle River, NJ: Prentice Hall.

Campbell, D. E. (1979). Interior office design and visitor response. Journal of Applied Psychology, 64(6), 648-653.

Cappella, J. N. (1981). Mutual influence in expressive behavior: Adult-adult and infant-adult dyadic interaction. Psychological Bulletin, 89(1), 101-132.

Cegala, D. J., Gade, C., Lenzmeier Broz, S., \& McClure, L. (2004). Physicians' and patients' perceptions of patients' communication competence in a primary care medical interview. Health Communication, 16(3), 289-304.

Chaikin, A. L., \& Derlega, V. J. (1974). Variables affecting the appropriateness of self-disclosure. Journal of Consulting and Clinical Psychology, 42(4), 588593.

Chaikin, A. L., Derlega, V. J., \& Miller, S. J. (1976). Effects of room environment and self-disclosure in a counseling analogue. Journal of Counseling Psychology, 23 (5), 479-481.

Chelune, G. J. (1975). Self-disclosure: An elaboration of its basic dimensions. Psychological Reports, 36(1), 79-85.

Cohen, B. N., \& Schwartz, R. C. (1997). Environmental factors and clients' selfdisclosure in counseling. Psychological Reports, 81(31), 931-934.

Collins, N. L., \& Miller, L. C. (1994). Self-disclosure and liking: A meta-analytic review. Psychological Bulletin, 116(3), 457-475. 
Cozby, P. C. (1973). Self-disclosure: A literature review. Psychological Bulletin, 79(2), 73-91.

Cunningham, M. R., Steinberg, J., \& Grev, R. (1980). Wanting to and having to help: Separate motivations for positive mood and guilt-induced helping. Journal of Personality and Social Psychology, 38(2), 181-192.

Dailey, L. (2004). Navigational web atmospherics: Explaining the influence of restrictive navigation cues. Journal of Business Research, 57(7), 795-803.

Daily DNS Changes and Web Hosting Activity (2013). Key figures and tables. Retrieved on May 3, 2013, http://dailychanges.com.

Daves, W. F., \& Swaffer, P. W. (1971). Effect of room size on critical interpersonal distance. Perceptual and Motor Skills, 33(31), 926-926.

De Vries, P., \& Pruyn, A. Th. H. (2007). Source salience and the persuasiveness of peer recommendations: The mediating role of social trust. Lecture Notes in Computer Science, 4744, 164-175.

Derksen, J. J. L., de Mey, H. R. A., Sloore, H., \& Hellenbosch, G. (1993). MMPI-2: Handleiding bij afname, scoring en interpretatie. Nijmegen: PEN Test Publishers.

Derlega, V. J., \& Grzelak, J. (1979). Appropriateness of self-disclosure. In G. J. Chelune (Ed.), Origins, patterns and implications of openness in interpersonal relationships (pp. 151-176). San Francisco: Jossey-Bass.

Derlega, V. J., Winstead, B. A., Mathews, A., \& Braintman, A. L. (2008). Why does someone reveal highly personal information? Attributions for and against self disclosure in close relationships. Communication Research Reports, 25(2), 115-130.

Dijkstra, K., Pieterse, M. E., \& Pruyn, A. T. H. (2006). Physical environmental stimuli that turn healthcare facilities into healing environments through psychologically mediated effects: Systematic review. Journal of Advanced Nursing, 56(2), 166-181.

Dijkstra, K., Pieterse, M. E., \& Pruyn, A. T. H. (2008). Individual differences in reactions towards color in simulated healthcare environments. The role of stimulus screening ability. Journal of Environmental Psychology, 28(3), 268277.

Dindia, K., \& Allen, M. (1992). Sex differences in self-disclosure: A meta-analysis. Psychological Bulletin, 112(1), 106-124.

Dosey, M. A., \& Meisels, M. (1969). Personal space and self-protection. Journal of Personality and Social Psychology, 11(2), 93-97.

Duggan, A. (2006). Understanding interpersonal communication processes across health contexts: Advances in the last decade and challenges for the next decade. Journal of Health Communication, 11, 93-108.

Durak, A., Olgonturk, N. C., Yener, C., Guvenc, D., \& Gurcinar, Y. (2007). Impact of lighting arrangements and illuminances on different impressions of a room. Building and Environment, 42(10), 3476-3482. 
Dutton, D. (2009). The art instinct: Beauty, pleasure, \& human evolution. New York: Bloomsbury Press.

Easterbrook, J. A. (1959). The effect of emotion on cue utilization and the organization of behavior. Psychological Review, 66(3), 183-201.

Epstein, R., \& Kanwisher, N. (1998). A cortical representation of the local visual environment. Nature 392(6676), 598-601.

Eroglu, S. A., Machleit, K. A., \& Davis, L. M. (2001). Atmospheric qualities of online retailing: A conceptual model and implications. Journal of Business Research, 54(2), 177-184.

Evans, G. W. (1979). Behavioral and physiological consequences of crowding in humans. Journal of Applied Social Psychology, 9, 27-46.

Evans, G. W., Lepore, S. J., Shejwal, B. R., \& Palsane, M. N. (1998). Chronic residential crowing and children's well-being: An ecological perspective. Child Development, 69, 1514-1523.

Flynn, J. E., Spencer, T. J., Martyniuk, O., \& Hendrick, C. (1973). Interim study of procedures for investigating the effect of light on impression and behavior. Journal of the Illuminating engineering Society, 3(1), 84-94.

Fogg, B. J., Soohoo, C., Danielson, D. R., Marable, L., Stanford, J., \& Tauber, E. R. (2003, June). How do users evaluate the credibility of web sites? A study with over 2,500 participants. In Proceedings of the 2003 conference on designing for user experiences (DUX'03), (pp. 1-15). ACM.

Forgas, J. P. (2011). Affective influences on self-disclosure. Mood effects on the intimacy and reciprocity of disclosing personal information. Journal of Personality and Social Psychology, 100(3), 449-461.

Friedman, R. S., \& Förster, J. (2010). Implicit affective cues and attentional tuning: An integrative review. Psychological Bulletin, 136(5), 875-893.

Gefen, D., \& Straub, D. W. (2004). Consumer trust in B2C e-commerce and the importance of social presence. Experiments in e-products and e-services. Omega, 32(6), 407-424.

Gifford, R. (1988). Light, decor, arousal, comfort and communication. Journal of Environmental Psychology, 8(3), 177-189.

Gosling, S. D., Ko, S. J., Mannarelli, T., \& Morris, M. E. (2002). A room with a cue, Personality judgments based on offices and bedroom. Journal of Personality and Social Psychology, 82(3), 379-398.

Greene, L. R. (1977). Effects of verbal evaluation feedback and interpersonal distance on behavioural compliance. Journal of Counseling Psychology, 24(1), 10-14.

Gunawardena, C. N., \& Zittle, F. J. (1997). Social presence as a predictor of satisfaction within a computer-mediated conferencing environment. American Journal of Distance Education, 11(3), 8-26.

Hall, E. T. (1965). The silent language. New York: Doubleday.

Hall, E. T. (1966). The hidden dimension. New York: Doubleday. 
Halpern, T. P. (1977). Degree of client disclosure as a function of past disclosure, counselor disclosure, and counselor facilitativeness. Journal of Counseling Psychology, 24, 41-47.

Harrington, J., Noble, L. M., \& Newman, S. P. (2004). Improving patients' communication with doctors: a systematic review of intervention studies. Patient Education and Counseling, 52(1), 7-16.

Harris, P.B., Ross, C., McBride, G., \& Curtis, L. (2002). A place to heal: Environmental sources of satisfaction among hospital patients. Journal of Applied Social Psychology, 32(6),1276-1299.

Haytko, D. L., \& Baker, J. (2004). It's all at the mall: Exploring adolescent girls' experiences. Journal of Retailing, 80(1), 67-83.

Hediger, H. (1955). The psychology and behaviour of animals in zoos and circuses (G. Sircom, Trans. 1968 ed.). New York: Dover.

Hendrick, C., Martyniuk, O., Spencer, T. J., \& Flynn, J. E. (1977). Procedures for investigating the effect of light on impression. Environment and Behavior, 9(4), 491-510.

Hidayetoglu, M. L., Yildirim, K., \& Akalin, A. (2012). The effects of color and light on indoor wayfinding and the evaluation of the perceived environment. Journal of Environmental Psychology, 32(1), 50-58.

Hinson, J. A., \& Swanson, J. L. (1993). Willingness to seek help as a function of selfdisclosure and problem severity. Journal of Counseling \& Development, 71(4), 465-470.

Hofstede, G., Hofstede, G. J., \& Minkov, M. (2010). Cultures and Organizations: Software of the Mind. New York: McGraw-Hill.

Howell, A., \& Conway, M. (1990). Perceived intimacy of expressed emotion. Journal of Social Psychology, 130, 467-476.

Høgh-Olesen, H. (2008). Human spatial behaviour. The spacing of people, objects and animals in six cross-cultural samples. Journal of Cognition and Culture, 8(3-4), 245-280.

Hui, M. K., \& Bateson, J. E. G. (1991). Perceived control and the effects of crowding and consumer choice on the service experience. Journal of Consumer Research, 18(2), 174-184.

Hur, M., Nasar, J. L., \& Chun, B. (2010). Neighborhood satisfaction, physical and perceived naturalness and openness. Journal of Environmental Psychology, 30(1), 52-59.

Ignatius, E., \& Kokkonen, M. (2007). Factors contributing to verbal self-disclosure. Nordic Psychology, 59, 362-391.

IJzerman, H., \& Semin, G. R. (2010). Temperature perceptions as a ground for social proximity. Journal of Experimental Social Psychology, 46(6), 867-873.

Joinson, A. N. (2001). Knowing me, knowing you: Reciprocal self-disclosure in internet-based surveys. CyberPsychology and Behavior, 4(5), 578-591. 
Joinson, A. N., Paine, C., Buchanan, T., \& Reips, U-D. (2008). Measuring selfdisclosure online: Blurring and non-response to sensitive items in web-based surveys. Computers in Human Behavior, 24(5), 2158-2171.

Jourard, S. M., \& Friedman, R. (1970). Experimenter-subject "distance" and selfdisclosure. Journal of Personality and Social Psychology, 15(3), 278-282.

Jourard, S. M., \& Lasakow, P. (1958). Some factors in self-disclosure. Journal of Abnormal and Social Psychology, 56, 91-98.

Jung, J. (2001). Psychology of Alcohol and Other Drugs. A Research Perspective. Newbury Park, CA: Sage.

Kahn, J. H., Lamb, D. H., Champion, D., Eberle, J. A., \& Schoen, K. A. (2002). Disclosing versus concealing distressing information: Linking self-reported tendencies to situational behavior. Journal of Research in Personality, 36, 531-538.

Kaplan, R., \& Kaplan, S. (1988). Where cognition and affect meet: A theoretical analysis of preference. In Nasar, J. (Ed). Environmental aesthetics. Theory, research, and applications (pp. 56-63). Cambridge: University Press.

Kaufman, E. (Ed.). (1981). IES Lighting Handbook. Application Volume. New York: Illuminating Engineering Society of North America.

Kim, J., Fiore, A. M., \& Lee, H.-H. (2007). Influences of online store perception, shopping enjoyment, and shopping involvement on consumer patronage behavior towards an online retailer. Journal of Retailing and Consumer Services, 14(2), 95-107.

Komar, V., Wypijewski, J., \& Melamid, A. (1997). Painting by numbers; Komar and Melamid's scientific guide to art. Berkeley, CA: University of California Press.

Koo, D.-M., \& Ju, S.-H. (2010). The interactional effects of atmospherics and perceptual curiosity on emotions and online shopping intention. Computers in Human Behavior, 26(3), 377-388.

Kwallek, N. (1996). Office wall color: An assessment of spaciousness and preference. Perceptual and Motor Skills, 83(1), 49-50.

Lakoff, G., \& Johnson, M. (1980). Metaphors we live by. Chicago: University of Chicago Press.

Lecomte, C., Bernstein, B. L., \& Dumont, F. (1981). Counseling interactions as a function of spatial-environmental conditions. Journal of Counseling Psychology, 28(6), 536-539.

Levav, J., \& Zhu, R. (2009). Seeking freedom through variety. Journal of Consumer Research, 36(4), 600-610.

Machleit, K. A., Eroglu, S. A., \& Mantel, S. P. (2000). Perceived retail crowding and shopping satisfaction: What modifies this relationship? Journal of Consumer Psychology, 9(1), 29-42.

Manav, B. (2007). An experimental study on the appraisal of the visual environment at offices in relation to colour temperature and illuminance. Building and Environment, 42(2), 979-983. 
Mandel, N., \& Johnson, E. J. (2002). When web pages influence choice: Effects of visual primes on experts and novices. Journal of Consumer Research: 29(2), 235-245.

Martin, K. A., \& Leary, M. R. (2001). Self-presentational determinants of health risk behavior among college freshmen. Psychology \& Health, 16(1), 17-27.

Maslow, A. H., \& Mintz, N. L. (1956). Effects of aesthetic surroundings: Initial effects of three aesthetic conditions upon perceiving 'energy' and 'wellbeing' in faces. Journal of Psychology, 4, 247-254.

Mathews, A., Derlega, V. J., \& Morrow, J. (2006). What is highly personal information and how is it related to self-disclosure decision-making? The perspective of college students. Communication Research Reports, 23(2), 85-92.

Mehrabian, A., \& Russell, J. A. (1974). An approach to environmental psychology. Cambridge: M.I.T. Press.

Meier, B. P., \& Robinson, M. D. (2004). Why the sunny side is up. Associations between affect and vertical position. Psychological Science, 15(4), 243-247.

Meyers-Levy, J., \& Zhu, R. (2007). The influence of ceiling height: The effect of priming on the type of processing that people use. Journal of Consumer Research, 34(2), 174-186.

Miwa, Y., \& Hanyu, K. (2006). The effects of interior design on communication and impressions of a counselor in a counseling room. Environment and Behavior, 38(4), 484-502.

Morton, T. L. (1978). Intimacy and reciprocity of exchange: A comparison of spouses and strangers. Journal of Personality and Social Psychology, 36(1), 72-81.

Nilsson, D. E., Strassberg, D. S., \& Bannon, J. (1979). Perceptions of counselor self disclosure: An analogue study. Journal of Counseling Psychology, 26(5), 399404.

Oberfeld, D., Hecht, H., \& Gamer, M. (2010). Surface lightness influences perceived room height. The Quarterly Journal of Experimental Psychology, 63(10), 1999-2011.

Okken, V. S., van Rompay, T. J. L., \& Pruyn, A. Th. H. (2012). Exploring space in the consultation room: Environmental influences during patient-physician interaction. Journal of Health Communication, 17(4), 397-412.

Okken, V. S., van Rompay, T. J. L., \& Pruyn, A. Th. H. (2013). Room to move: on spatial constraints and self-disclosure during intimate conversations. Environment \& Behavior, 45(6), 737-760.

Olsen, G. D., Pracejus, J. W., \& O'Guinn, T. C. (2012). Printing advertising: White space. Journal of Business Research, 65(6), 855-860.

Omarzu, J. (2000). A disclosure decision model: Determining how and when individuals will self-disclose. Personality and Social Psychology Review, 4(2), 174-185. 
Palmstierna, T., Huitfeldt, B., \& Wistedt, B. (1991). The relationship of crowding and aggressive behavior on a psychiatric intensive care unit. Hospital and Community Psychiatry, 42, 1237-1240.

Patterson, M. L. (1973). Compensation in nonverbal immediacy behaviors: A review. Sociometry, 36(2), 237-252.

Pennebaker, J. W., \& Beal, S. K. (1986). Confronting a traumatic event; Toward an understanding of inhibition and disease. Journal of Abnormal Psychology, 95, 274-281.

Pennebaker, J. W., Kiecolt-Glaser, J. K., \& Glaser, R. (1988). Disclosure of traumas and immune function: Health implications for psychotherapy. Journal of Consulting and Clinical Psychology, 56, 239-245.

Pontell, H. N., \& Welsh, W. N. (1994). Incarceration as a deviant form of social control: Jail overcrowding in California. Crime and Delinquency, 40, 18-36.

Pracejus, J. W., Olsen, G. D., \& O'Guinn, T. C. (2006). How nothing became something, White space, rhetoric, history, and meaning. Journal of Consumer Research, 33(1), 82-90.

Pressly, P. K., \& Heesacker, M. (2001). The physical environment and counseling: A review of theory and research. Journal of Counseling and Development, 79(2), 148-160.

Remland, M. S., Jones, T. S., \& Brinkman, H. (1995). Interpersonal distance, body orientation, and touch: Effects of culture, gender, and age. Journal of Social Psychology, 135, 281-297.

Richard, M.-O. (2005). Modeling the impact of internet atmospherics on surfer behavior. Journal of Business Research, 58(12), 1632-1642.

Riley, G. D., Cozby, P. C., White, G. D., \& Kjos, G. L. (1983). Effect of therapist expectations and need for approval on self-disclosure. Journal of Clinical Psychology, 39(2), 221-226.

Rosen, D. E., \& Purinton, E. (2004). Website design: Viewing the web as a cognitive landscape. Journal of Business Research, 57(7), 787-794.

Roter, D. L., \& Hall, J. A. (1987). Physicians' interviewing styles and medical information obtained from patients. Journal of General Internal Medicine, 2(5), 325-329.

Rotter, J. B. (1980). Interpersonal trust, trustworthiness, and gullibility. American Psychologist, 35(1), 1-7.

Sadalla, E. K., \& Oxley, D. (1984). The perception of room size: The rectangularity illusion. Environment and Behavior, 16(3), 394-405.

Sapolsky, R. M. (1998). Why zebras don't get ulcers. An updated guide to stress, stress-related diseases, and coping. New York: Freeman and Company.

Schiffenbauer, A., \& Schiavo, R. S. (1976). Physical distance and attraction: An intensification effect. Journal of Experimental Social Psychology, 12(3), 274282.

Sommer, R. (1959). Studies in personal space. Sociometry, 22(3), 247-260. 
Sommer, R. (2007). Personal space; updated. The behavioral basis of design. Bristol: Bosko Books.

Stamps, A. (1990). Use of photographs to simulate environments: A meta-analysis. Perceptual and Motor Skills, 71(3), 907-913.

Stamps, A. E. (2010). Effects of permeability on perceived enclosure and spaciousness. Environment and Behavior, 42(6), 864-886.

Stamps, A. E. (2011). Effects of area, height, elongation, and color on perceived spaciousness. Environment and Behavior, 43(2), 252-273.

Stamps, A. E., \& Krishnan, V. V. (2006). Spaciousness and Boundary Roughness. Environment and Behavior, 38(6), 841-872.

Strassberg, D. S., Anchor, K. N., Gabel, H., \& Cohen, B. (1978). Client self-disclosure in short-term psychotherapy. Psychotherapy: Theory, Research and Practice, 15(2), 153-157.

Strassberg, D., Roback, H., D’Antonio, M., \& Gabel, H. (1977). A critical and selective review of the clinical literature. Comprehensive Psychiatry, 18(1), 31-39.

Strong, E. K. (1926). Value of white space in advertising. Journal of Applied Psychology, 10(1), 107-116.

Sundstrom, E. (1975). An experimental study of crowding: Effects of room size, intrusion, and goal blocking on nonverbal behavior, self-disclosure, and selfreported stress. Journal of Personality and Social Psychology, 32(4), 645654.

Swan, J. E., Richardson, L. D., \& Hutton, J. D. (2003). Do appealing hospital rooms increase patient evaluations of physicians, nurses, and hospital services? Health Care Management Review, 28(3), 254-264.

Taylor, S. H., \& Gill, S. J. (1983). Professional disclosure in the counseling profession, A review of the literature. Patient Education and Counseling, 5(1), 35-40.

Teven, J. J., \& Comadena, M. E. (1996). The effects of office aesthetic quality on students' perceptions of teacher credibility and communicator style. Communication Research Reports, 13(1), 101-108.

Thiel, P., Harrison, E. D., \& Alden, R. S. (1986). The perception of spatial enclosure as a function of the position of architectural surfaces. Environment and Behavior, 18(2), 227-245.

Turley, L. W., \& Milliman, R. E. (2000). Atmospheric effects on shopping behavior: A review of the experimental evidence. Journal of Business Research, 49(2), 193-211.

Ulrich, R. S. (1995). Effects of interior design on wellness: Theory and recent scientific research. In Marberry, S.O. (Ed.). Innovations in healthcare design (pp. 88-104). New York: Van Nostrand Reinhold. 
Ulrich, R., Zimring, C., Zhu, X., DuBose, J., Seo, H. B., Choi, Y. S. , Quan, X., \& Joseph, A. (2008) A review of the research literature on evidence-based design. Health Environments Research \& Design Journal, 1(3), 61-125.

Valdez, P., \& Mehrabian, A. (1994). Effects of color on emotions. Journal of Experimental Psychology: General, 124(4), 394-409.

Van Rompay, T. J. L., de Vries, P. W., \& van Venrooij, X. G. (2010). More than words: On the importance of picture-text congruence in the online environment. Journal of Interactive Marketing, 24(1), 22-30.

Van Rompay, T. J. L., Galetzka, M., Pruyn, A. T. H., \& Moreno-Garcia, J. (2008). Human and spatial dimensions of retail density: The role of perceived control revisited. Psychology \& Marketing, 25 (4), 319-335.

Van Rompay, T. J. L., \& Tanja-Dijkstra, K. (2010). Directions in healthcare research: Pointers from retailing and services marketing. Health Environments Research \& Design Journal, 3(3), 87-100.

Verhoeven, J. W. M., van Rompay, T. J. L., \& Pruyn, A. T. H. (2007). Let your workspace speak for itself: The impact of material objects on impression formation and service quality perception. In G. J. Fitzsimons \& V. G. Morwitz (Eds.), Advances in Consumer Research (Vol. 34, pp. 669-674). Duluth, MN: Association for Consumer Research.

Walker, K. L., Arnold, C. L., Miller-Day, M., \& Webb, L. M. (2001). Investigating the physician-patient relationship: Examining emerging themes. Health Communication, 14(1), 45-68.

Ward, J. C., Bitner, M. J., \& Barnes, J. (1992). Measuring the prototypicality and meaning of retail environments. Journal of Retailing, 68(2), 194-213.

Wiederman, M. W., \& Sansone, R. A. (1999). Sexuality training for professional psychologists: A national survey of training directors of doctoral programs and predoctoral internships. Professional Psychology: Research and Practice, 30(3), 312-317.

Williams, L. E., \& Bargh, J. A. (2008). Keeping one's distance: The influence of spatial distance cues on affect and evaluation. Psychological Science, 19(3), 302-308.

Wyer, N. A., \& Calvini, G. (2011). Don't sit so close to me: Unconsciously elicited affect automatically provokes social avoidance. Emotion, 11(5), 1230-1234.

Yildirim, K., \& Akalin-Baskaya, A. (2007). Perceived crowding in a café/restaurant with different seating densities. Building and Environment, 42(9), 34103417.

Yildirim, K., Akalin-Baskaya, A., \& Hidayetoglu, M. L. (2007). Effects of indoor color on mood and cognitive performance. Building and Environment, 42(9), 32333240.

Zandbelt, L. C., Smets, E. M. A., Oort, F. J., Godfried, M. H., \& De Haes, H. C. J. M. (2007). Patient participation in the medical specialist encounter: Does 
physicians' patient-centred communication matter? Patient Education and Counseling, 65(3), 396-406. 


\section{Samenvatting}

(Summary in Dutch) 
Het communiceren van persoonlijke informatie aan anderen wordt ook wel zelfonthulling (self-disclosure) genoemd. Zelfonthulling betreft informatie die gevoelig ligt en niet zomaar met de hele wereld wordt gedeeld. Hoewel bij zelfonthulling al snel gedacht wordt aan één-op-één gesprekken hoeft dit niet altijd het geval te zijn. Het kan namelijk ook plaatsvinden tegenover meerdere mensen, tijdens het opschrijven van persoonlijke informatie die later door iemand anders zal worden gelezen en ook tijdens online interactie waarbij je persoonlijke informatie vrijgeeft. Het voordeel van het delen van persoonlijke informatie is dat dit een stress-verlagend effect kan hebben, de band met een andere persoon kan versterken en zelfs een positief effect kan hebben op de gezondheid. Er zitten echter ook nadelen aan het delen van gevoelige informatie met anderen, zoals afgewezen worden door de ander, verlaging van persoonlijke integriteit of controleverlies. Hieruit volgt logischerwijs dat zelfonthulling vaker plaatsvindt tegenover goede vrienden, familieleden en met name de partner dan tegenover vage bekenden en vreemden.

Er is echter nog een andere situatie waarin zelfonthulling van belang is; namelijk in de dienstverleningssector. De dienstverlener is voor een groot deel afhankelijk van de informatie van zijn cliënt om te bepalen wat deze van hem verlangt. Dus, hoe beter de cliënt in staat is om zijn verhaal te doen, hoe beter de dienstverlener in staat is om de cliënt te helpen. Een voorbeeld hiervan is het contact tussen artsen en therapeuten enerzijds en patiënten anderzijds. De arts of therapeut is voor een groot deel immers afhankelijk van de mondelinge informatie van de patiënt voor het stellen van een diagnose. Wanneer een patiënt meer persoonlijke informatie durft te delen, heeft de arts een completer beeld van de klachten en kan hij wellicht een betere diagnose stellen en een beter passende behandeling voorschrijven. Daarnaast kan zelfonthulling ook helpen om er bijvoorbeeld achter te komen of een patiënt zijn medicatie wel volgens voorschrift slikt. Zo ligt bij veel welvaartsziekten de verantwoordelijkheid voor het behandeltraject volledig in handen van de patiënt en vindt contact met de arts grotendeels plaats in de vorm van een periodiek gesprek waarin de arts de vooruitgang evalueert.

Wanneer iemand geen zelfonthulling wil vertonen in gesprek met een goede vriend, leidt dit soms tot het einde van het gesprek, maar veel vaker leidt het tot een verandering van gespreksonderwerp. Tijdens gesprekken met een dienstverlener is het veranderen van gespreksonderwerp echter erg onwaarschijnlijk. In plaats daarvan zal de dienstverlener blijven doorvragen om de benodigde informatie los te krijgen uit de cliënt. Als resultaat daarvan zal de cliënt zich steeds minder op zijn gemak voelen en het gesprek zal steeds stroever verlopen. Het valt te verwachten dat zo'n gesprek een zeer negatieve ervaring is voor de cliënt en dat het de bereidheid tot terugkomen bij deze dienstverlener verlaagt. Daarom is het van 
belang om te onderzoeken hoe zelfonthulling kan worden gestimuleerd, in zowel fysieke als online service omgevingen.

Tijdens een gesprek zijn er verschillende factoren aan te wijzen die van invloed zijn op de bereidheid tot het vertonen van zelfonthulling. Natuurlijk is zelfonthulling in eerste instantie afhankelijk van de beide personen aanwezig tijdens het gesprek. Bij de persoon zelf speelt de gemoedstoestand een grote rol; in vrolijke, ontspannen toestand zal deze makkelijker praten dan in een bezorgde, gespannen toestand. Daarnaast verschillen mensen ook qua persoonlijkheid in hun algemene bereidheid om gesprekken aan te gaan met mensen en in het gemak waarmee ze met anderen praten. Verder hebben ook de eigenschappen van de gesprekspartner, zoals geslacht en leeftijd, invloed op de hoeveelheid zelfonthulling, maar ook de bekendheid met de gesprekspartner. Ook spelen de gespreksinhoud en de wederzijdse bijdragen een rol. Het blijkt dat een hogere mate van reciprociteit tijdens een gesprek leidt tot meer zelfonthulling, vooral tijdens een gesprek met een onbekende gesprekspartner.

Samengevat kunnen we stellen dat de context waarbinnen zelfonthulling tijdens dienstverlening plaatsvindt de zelfonthulling van de cliënt allerminst bespoedigt; angst en onzekerheid over de te delen informatie gaat dikwijls samen met een gevoel van dreiging, de dienstverlener wordt niet gezien als een goede vriend en door de aard van het gesprek zal er altijd sprake zijn van een ongelijke 'verdeling' wanneer het aankomt op zelfonthulling. Maar, naast de aanwezige personen en het gespreksonderwerp is er nog een 'vierde' variabele die gebruikt kan worden om zelfonthulling van patiënten te stimuleren: de omgeving waarin het gesprek plaatsvindt. De vraag die centraal staat in dit proefschrift is in hoeverre omgevingsfactoren kunnen worden ingezet om zelfonthulling te verbeteren.

Onderzoek toont aan dat omgevingsfactoren zoals de architectuur van een ruimte, de indeling van het meubilair, verlichtingsinstellingen, gebruikte kleuren, aanwezige geuren en muziek van invloed kunnen zijn op hoe we ons voelen en gedragen. Als verklaring hiervoor wordt vaak gesteld dat dit afhangt van de mate waarin een persoon zich op zijn gemak voelt in een bepaalde ruimte. Echter, de mate waarin iemand zich op zijn gemak voelt wordt sterk bepaald door iemands persoonlijke smaak en voorkeuren. Het lijkt dus erg lastig om op basis van deze verklaring ruimtes te ontwerpen die voldoen aan iedereens wensen. Daarom wordt in dit proefschrift een alternatieve verklaring geïntroduceerd en getoetst, namelijk dat de ervaren ruimtelijkheid een onderliggende factor is die de invloed van omgevingsfactoren op zelfonthulling kan verklaren. De ervaren ruimtelijkheid van een kamer is namelijk meestal een van de eerste dingen die opvalt bij het betreden van een onbekende kamer. 
Inzicht in hoe ons gedrag wordt beïnvloed door de ervaren ruimtelijkheid volgt uit onderzoek naar de invloed van plafondhoogte. Een hoog plafond bleek gevoelens van vrijheid te activeren, wat vervolgens leidde tot meer creatieve strategieën bij probleemoplossingstaken. In vergelijkbaar onderzoek werd in een supermarkt de invloed van ruimtelijkheid in gangpaden onderzocht. De resultaten van dit onderzoek laten zien dat een smal gangpad zorgde voor een opgesloten gevoel. Dit leidde vervolgens tot een behoefte aan vrijheid wat zich in de supermarkt manifesteerde in een meer gevarieerde productkeuze. Deze onderzoeksresultaten suggereren dat het beperken van de fysieke ruimte gevoelens van beperkte psychologische ruimte oproept. Dit negatieve gevoel van beperking verlaagt vervolgens de intentie om het gewenste gedrag te vertonen, een proces dat ook wel wordt geduid met de term 'reactance'. Vertaald naar de huidige context valt dus te verwachten dat pogingen van een dienstverlener om de cliënt meer zelfonthulling te laten vertonen niet zullen slagen wanneer de ervaren ruimtelijkheid binnen de gesprekssetting niet voldoet aan de wensen van de cliënt.

Om overzicht aan te brengen in de vele omgevingsfactoren die aanwezig kunnen zijn in een omgeving wordt in dit proefschrift een taxonomie gebruikt die onderscheid maakt tussen vier categorieën; namelijk architectuurfactoren (bijv. kamerbreedte, plafondhoogte, kamergrootte), interieurfactoren (bijv. meubelopstelling, inrichting, losse accessoires), atmosferische factoren (bijv. licht, kleur, geur en temperatuur) en, met betrekking tot online communicatie, website design factoren. In de vier empirische hoofdstukken die volgen, zullen factoren uit elk van deze categorieën worden getoetst.

\section{Hoofdstuk 3}

In het eerste empirische hoofdstuk van dit proefschrift worden twee onderzoeken beschreven naar de invloed van omgevingsfactoren op de intentie tot zelfonthulling tijdens patiënt-arts communicatie. Kamergrootte en interpersoonlijke afstand tussen patiënt en arts werden gevarieerd in foto's van een spreekkamer van een arts en kregen daarnaast een scenario te lezen. Experiment 3.1 toonde aan dat de manipulaties invloed hadden op de ervaren ruimtelijkheid. Daarnaast bleek dat de ervaren ruimtelijkheid invloed had op de mate van comfort en de intentie tot zelfonthulling. De resultaten lieten ook zien dat afhankelijk van de persoonlijkheidseigenschap gereserveerdheid effecten van ruimtelijkheid variëren; mensen met weinig gereserveerdheid beoordeelden de grote kamer ruimer dan de kleine kamer en hadden bovendien een hogere intentie tot zelfonthulling in de grote kamer. Preferentie en intentie tot zelfonthulling bij mensen met weinig gereserveerdheid verschilden echter niet; een bevinding die laat zien dat effecten van ruimtelijkheid op zelfonthulling onderhevig zijn aan persoonsgebonden invloeden. 
In Experiment 3.2 werd, naast dezelfde omgevingsmanipulaties, ook de toon van het gesprek gevarieerd in het voorgelegde scenario door een positieve en een negatieve variant te creëren. De resultaten van dit tweede onderzoek kwamen in grote lijnen overeen met de resultaten van Experiment 3.1. Daarnaast werd aangetoond dat patiëntbehoeften met betrekking tot ervaren ruimtelijkheid varieerden afhankelijk van het gesprek; in een gesprek met lage dreiging was in een kleine kamer de intentie tot zelfonthulling hoger bij een grotere afstand, terwijl in een grote kamer deze intentie hoger was bij een kleinere afstand. In een positief gesprek was er dus behoefte aan toenadering, onder voorwaarde dat de kamer groot genoeg was. In een gesprek met hoge dreiging werd een algehele voorkeur voor meer ruimte gevonden; zowel een grotere kamer als een grotere interpersoonlijke afstand had de voorkeur.

\section{Hoofdstuk 4}

Het vierde hoofdstuk beschrijft een simulatie onderzoek waarin de manipulaties uit het vorige hoofdstuk (kamergrootte en bureaugrootte) worden getoetst tijdens lifestyle interviews. Twee vergelijkbare kamers van verschillende formaten werden gebruikt, met daarin of een groot bureau of een klein bureau. Het interview bevatte vragen over verschillende persoonlijke onderwerpen, namelijk; alcohol- en drugsgebruik, seksualiteit en emoties. De interviews werden opgenomen op video zodat het non-verbale gedrag ook kon worden onderzocht.

De resultaten bevestigen dat zowel bureaugrootte als kamergrootte invloed had op de ervaring en het gemak van zelfonthulling van de deelnemers en dat deze relatie werd gemedieerd door ervaren ruimtelijkheid. Een interessante bevinding was dat de behoefte aan ruimtelijkheid varieerde per onderwerp. Bureaugrootte (ofwel interpersoonlijke afstand) was het meest van belang bij de vragen met betrekking tot seksualiteit en kamergrootte was het belangrijkst tijdens de onderwerpen alcohol- en drugsgebruik en emoties. Daarnaast bleek dat non-verbaal gedrag door deelnemers werd ingezet om een ruimtelijkere opstelling te creëren (door bijvoorbeeld achterover te leunen of minder oogcontact te maken) wanneer de situatie te benauwend of te intiem is.

\section{Hoofdstuk 5}

In dit hoofdstuk wordt een experiment besproken dat de invloed van atmosferische factoren (zoals verlichtingsinstellingen of kleurgebruik) op de mate van zelfonthulling onderzoekt. In dit experiment werd de helderheid van een consultatiekamer gemanipuleerd en werden de ervaren ruimtelijkheid en de intentie tot zelfonthulling onderzocht. Ook in deze studie werd de dreiging van het gesprek gemanipuleerd. 
Hoewel er geen hoofdeffecten werden gevonden voor de helderheid, laat de gevonden interactie tussen helderheid en de gecommuniceerde dreiging wel hetzelfde patroon zien als de eerder genoemde resultaten in de voorgaande twee hoofdstukken. Tijdens een positief gesprek werd geen effect van helderheid gevonden op zelfonthulling, wat suggereert dat de behoefte aan ruimtelijkheid in een positief gesprek niet saillant is. Tijdens een negatief gesprek echter, werd wederom wel een effect van ervaren ruimtelijkheid (helderheid) gevonden; in de heldere kamer was de intentie tot zelfonthulling hoger dan in de donkere kamer. Dit houdt in dat bij een hoge dreiging het ervaren van te weinig ruimte inderdaad leidde tot een verlaagde intentie tot zelfonthulling. Daarnaast laten de resultaten zien dat helderheid ook patiënt oordelen over hun arts beïnvloedden; in de heldere kamer werd de arts aardiger gevonden dan in de donkere kamer. De relatie tussen de helderheid en het oordeel over de arts werd echter niet gemedieerd door de ervaren ruimtelijkheid, maar door de affectieve ervaring.

\section{Hoofdstuk 6}

Het laatste empirische hoofdstuk van dit proefschrift beschrijft twee onderzoeken waarin de invloed van de fysieke omgeving en de website omgeving worden onderzocht op online self-disclosure. In Onderzoek 6.1 wordt ingegaan op de vraag of het manipuleren van website design elementen (de ervaren interpersoonlijke afstand en de helderheid van het achtergrondscherm) invloed heeft op de ervaren ruimtelijkheid en op de mate van zelfonthulling tijdens een online consult met een huisarts. Deelnemers kregen een online chatprogramma te zien en moesten de vragen van de huisarts beantwoorden in het antwoordveld van het chatprogramma.

De resultaten toonden aan dat de online manipulaties invloed hadden op zowel ervaren ruimtelijkheid als de mate van zelfonthulling; een ruimer website design leidt tot meer zelfonthulling dan een minder ruime website design. Daarnaast bevestigen de resultaten wederom dat de aanwezigheid van dreiging de behoefte aan ruimte vergroot. Hoewel er een vergelijkbaar patroon was in de gevonden effecten voor ervaren ruimtelijkheid en zelfonthulling, was het mediërende effect van ervaren ruimtelijkheid echter niet significant. Daarom werd in Onderzoek 6.2 een andere mediator meegenomen, die sterk gerelateerd is aan de ervaren ruimtelijkheid, maar is toegespitst op online omgevingen, namelijk ervaren ruimte voor zelfexpressie (oftewel de mate waarin iemand het gevoel heeft dat hij zijn verhaal kwijt kan op de website). Daarnaast werd in Onderzoek 6.2 naast de effecten van de online omgeving ook gekeken naar de effecten van de fysieke omgeving, om te onderzoeken in hoeverre deze van invloed zijn tijdens interacties die plaatsvinden op een computerscherm. Verder werd onderzocht in hoeverre de manipulaties een effect hadden op reactance (een verlaagde intentie om het gewenste gedrag te vertonen die ontstaat als gevolg van het gevoel van opgelegde 
beperkingen of te weinig ruimte). Website design werd in deze studie gemanipuleerd door de hoeveelheid witte ruimte te manipuleren en de helderheid van de kamer werd gemanipuleerd door de verlichtingscondities van de kamer aan te passen.

De resultaten van dit tweede onderzoek laten zien dat de helderheid van de kamer van invloed is op de ervaren ruimtelijkheid; een helderdere kamer leidt tot meer ruimtelijkheid en als gevolg daarvan tot meer zelfonthulling en minder reactance. Dit bevestigt dat deelnemers meer bereid zijn om het gewenste gedrag te vertonen in een omgeving die ruimer overkomt. Verder laten de resultaten een vergelijkbaar resultaat zien voor website ontwerp. Een ruimer website design leidt tot meer ervaren ruimte voor zelfexpressie en als gevolg daarvan tot meer zelfonthulling en minder reactance. Samengevat laten de resultaten zien dat de effecten van de fysieke en de online omgeving sterk aan elkaar verbonden zijn. Daarnaast demonstreren de bevindingen dat, hoewel dienstverleners zelden tot nooit invloed hebben op de fysieke omgeving waarin de cliënt zich bevindt tijdens online interactie, een ruimer website design wellicht mogelijke negatieve effecten van suboptimale fysieke omstandigheden in termen van ruimtelijkheid kan beperken.

\section{Algemene conclusie}

De onderzoeken in dit proefschrift bevestigen de invloed van omgevingsfactoren op ons gevoel en gedrag. Alle onderzoeken laten zien dat omgevingsfactoren behorende tot elk van de vier categorieën uit de gepresenteerde taxonomie (in Hoofdstuk 2) in staat zijn om zelfonthulling te beïnvloeden. Daarnaast wordt het belang van ervaren ruimtelijkheid aangetoond. De introductie van deze mediator in de relatie tussen omgevingsfactoren en zelfonthulling geeft inzicht in het onderliggende proces dat de gevonden effecten veroorzaakt. De informatie kan bijdragen aan de kennis van architecten en ontwerpers van service omgevingen en wellicht meer inzicht bieden in omgevingsinvloeden dan de geïsoleerde effecten die gevonden zijn voor verschillende factoren.

Het is aangetoond dat ervaren ruimtelijkheid niet alleen wordt beïnvloed door de fysieke omgeving, maar ook door eventuele dreiging die aanwezig is tijdens het gesprek. Zo blijkt dat omgevingen minder ruim overkomen tijdens gesprekken met een negatieve of dreigende gesprekstoon dan tijdens gesprekken met een positievere gesprekstoon. Naast zelfonthulling bleek ook het non-verbale gedrag te worden beïnvloed door de ervaren ruimtelijkheid; effecten werden gevonden voor lichaamshouding en mate van oogcontact. De bevinding dat een toename van ervaren ruimtelijkheid leidt tot een afname in reactance bevestigt dat mensen meer bereid zijn om het gewenste gedrag, zelfonthulling, te vertonen in een omgeving die ruimer overkomt. Daarnaast suggereert dit effect dat niet alleen zelfonthulling, 
maar ook andere aspecten van communicatief of sociaal gedrag kunnen worden beïnvloed door de ervaren ruimtelijkheid.

Naast architecten en ontwerpers kunnen ook dienstverleners, die wellicht aanpassingen aanbrengen of objecten toevoegen aan de inrichting zonder zich bewust te zijn van de mogelijke gevolgen hiervan, profiteren van deze informatie. Daarnaast zou het dienstverleners kunnen helpen om zich bewust te zijn van het concept ervaren ruimtelijkheid en dat dit niet alleen wordt beïnvloed door de fysieke omgeving, maar ook door de toon van het gesprek. Dienstverleners zouden meer aandacht kunnen schenken aan het non-verbale gedrag van cliënten; onschuldige veranderingen in de lichaamshouding of mate van oogcontact die kunnen een aanwijzing zijn voor een behoefte aan meer ruimte. Wanneer hier meer rekening mee wordt gehouden en de behoefte aan ruimtelijkheid van cliënten bewuster wordt gerespecteerd, kan dit bijdragen aan het verbeteren van de mate van zelfonthulling van cliënten en aan het dienstverleningsproces in zijn geheel.

De uitgevoerde experimenten draagt bij aan onze kennis op het gebied van omgevingspsychologie en communicatie onderzoek. De experimenten laten zien dat de omgeving niet alleen een bijstander is, maar actief deelneemt aan de conversaties en invloed heeft op hoe we ons gedragen. De toevoeging van ervaren ruimtelijkheid als mediator biedt nieuwe inzichten in het verklaren van omgevingsinvloeden en kan hopelijk bijdragen aan het verder verbeteren van de onze gebouwde omgeving. 


\section{Dankwoord}

(Acknowledgements in Dutch) 
Een proefschrift schrijven doe je niet alleen. Zonder de begeleiding, de hulp, het gezelschap, de steunbetuigingen en de blijken van interesse van vele mensen om me heen, zou dit boekje er dus niet hebben gelegen. Ik wil iedereen bedanken die, op wat voor manier dan ook, (soms zelfs zonder het te weten) heeft bijgedragen aan de totstandkoming van mijn proefschrift, en een aantal mensen in het bijzonder.

Allereerst wil ik mijn promotor, Ad Pruyn, bedanken. Ad, bedankt dat je destijds dusdanig hard met je vuist op de tafel van de toenmalige decaan hebt geslagen dat mijn jaarcontract als onderzoeker werd verlengd met een promotieproject, wat uiteindelijk geleid heeft tot dit proefschrift. Zowel tijdens onze meetings over papers en het proefschrift als tijdens onze potjes Kolonisten van Catan werd er soms verhit gediscussieerd, maar dit kwam mijns inziens vooral doordat we het allebei erg serieus namen. Jij zorgde altijd dat de focus op de veelgenoemde 'rode draad' aanwezig bleef. Ik heb veel van je geleerd en wil je bedanken voor je begeleiding de afgelopen jaren. Ik wil Diane graag bedanken voor het lezen en verbeteren van een deel van dit proefschrift. Diane en Ad, bedankt voor jullie gastvrijheid en gezelligheid de afgelopen jaren. Ooit zal ik Catan van jullie winnen!

Daarnaast ben ik mijn dagelijks begeleider en co-promotor, Thomas van Rompay, veel dank verschuldigd. Thomas, dat ik met veel plezier terugkijk op de afgelopen jaren is voor een groot deel aan jou te danken. Jouw deur stond elke dag, letterlijk maar vooral ook figuurlijk, open en je maakte altijd tijd vrij voor een babbeltje, over het werk of over totaal andere dingen. Ik bewonder je positieve instelling en ik denk dat jouw positiviteit een goede tegenhanger was van mijn doemdenkerij. Ik ben nog altijd ontzettend jaloers op je mooie verhalende manier van schrijven en het gemak waarmee je de mooiste zinnen op papier weet te zetten. In de loop der tijd ben ik je meer als goede vriend gaan beschouwen dan als begeleider. Ik hoop dus ook dat mijn promotietijd slechts de basis zal zijn van een blijvende vriendschap met jou, Suzanne en Matisse. Ik kijk uit naar meer mooie zomeravonden in Enschede of Hengelo, waarbij de moscatel of asti uiteraard niet mag ontbreken!

Ik wil graag alle leden van mijn promotiecommissie bedanken voor de tijd en moeite die ze hebben genomen voor het lezen van mijn proefschrift en voor hun aanwezigheid bij mijn verdediging.

Mijn collega's van de UT wil ik bedanken voor de stimulerende werkomgeving en de vele leuke herinneringen. Ik heb me vanaf dag 1 welkom gevoeld bij MCP (en op de valreep ook nog even bij CMC) en bij collega's van andere vakgroepen. Ik denk met plezier terug aan de gezellige overleggen, lunches, borrels en pubquizzen. In het bijzonder wil ik de volgende mensen bedanken. Loes, het was erg fijn om een kamer met jou te mogen delen. Er is flink wat thee doorgegaan, ik moet nog steeds glimlachen als ik James voorbij zie komen en begin altijd te grinniken als ik "to the 
window, to the wall" hoor! Peter, wat fijn om met jou m'n fascinatie voor rare beessies te kunnen delen. Nog altijd als ik ergens een nieuw exemplaar tegenkom, leg ik het voor je vast (en ik wacht nog steeds op een foto van het 'lopend vuurtje'). Ik ben blij dat Stephanie en jij verhuisd zijn naar jullie prachtige boerderij in Boekelo zodat we nog eens bij elkaar op de koffie (borrel) kunnen komen. Bedankt voor alle hulp bij de onderzoeken, vooral voor je hulp bij Authorware ben ik je erg dankbaar! Sabrina, sometimes you catch a glimpse of a person and instantly know that you're going to be great friends. This happened at your first day at the UT. I can always count on you for lots of positivity and mindful advises. You're a star! Alexander, Joost, Jordy, Joris, Lex, Marieke, Martijn, Mirjam, Nathalie, Suzanne, Willem: Bedankt voor de leuke tijden!

Mijn paranimfen, Lisanne en Karin, ik vind het geweldig dat ik tijdens mijn verdediging word bijgestaan door twee van mijn hartsvriendinnen. Lisanne, onze vriendschap is begonnen tijdens de studie psychologie in Groningen, terwijl we worstelden met onze struikelblokken: klinische psychologie en geschiedenis van de psychologie. Dit is inmiddels 11 jaar geleden en in de tussentijd hebben we veel gedeeld. Samen hebben we onze propedeuses behaald, onze studies afgerond, de paus toegezwaaid, Starbucks ontdekt, Hera de zeehond geadopteerd, een ijsje gegeten bij de Trevi-fontein, over Portobello road geslenterd, en nog veel meer. Naast alle lol hebben we ook vele goede gesprekken gevoerd en niet alleen lief maar ook leed met elkaar gedeeld. Karin, mijn vriendschap met jou begon op de UT, als collega en kamergenoot. Samen fietsten we door weer en wind (en sneeuw), gingen we op vrijdagmiddag los op Pink en Rage against the Machine, versierden we muren met Hoops \& Yoyo, stonden we in de rij voor een handtekening van Ellen ten Damme, gilden we mee met Lady Gaga in de Gelredome, kwamen we er elk seizoen weer achter wie de mol was, speelden we super mario, reisden we af naar Land's end, picknickten we bij St. Michael's Mount en deden we nog veel meer hilarische dingen, waarbij de tranen ons vaak over de wangen liepen van het lachen. Basil zei ooit tegen ons: "you're having way too much fun", waarop wij in koor antwoordden: "there's no such thing as too much fun!". Ik kan op jou ook altijd een beroep doen als collega voor werk-gerelateerde tips en adviezen. Bedankt dat je er altijd voor me was de afgelopen jaren, dat je tijdens je literatuursearches nog altijd aan me denkt en interessante dingen doormailt, dat je mee-brainstormt over onderzoeksopzetten en kritisch meedenkt over statistische analyses en schrijfwerk. Vooral tijdens de laatste o zo zware loodjes heb ik echt ontzettend veel aan je gehad! Lisanne en Karin, de tijd heeft het ons geleerd: True friendship isn't about being inseperable, it's being separated and nothing changes. Mijn vriendschap met jullie betekent voor mij niet alleen dat we aan één blik voldoende hebben om in de lach te schieten, maar ook dat we feilloos aanvoelen wanneer de ander ons nodig heeft en al het andere op dat moment minder belangrijk is dan er voor elkaar te zijn. Bedankt dat jullie altijd voor me klaarstaan, no matter what! Jullie zijn me 
allebei ontzettend dierbaar en ik weet zeker dat we voor altijd in elkaars leven zullen blijven.

Buiten de UT heb ik ook het geluk om veel geweldige mensen om me heen te hebben met wie ik lief en leed kan delen. Bart en Ron, Vincent en Francien, Marjolijn en Remco, Gerrit en Anneke, Paul en Joyce, Wilco, Koen, Erna en Tet, Erna en Rene, Opa en Oma Kloek, Esther en Rene, Petra en Bass. Jullie zijn wellicht niet zo direct bij het onderzoek betrokken geweest, maar ieder van jullie heeft op zijn eigen manier meegeholpen aan de totstandkoming van dit proefschrift. Bedankt voor alle steun en liefde de afgelopen jaren. Bart en Ron, bij jullie voel ik me altijd thuis, wat fijn dat jullie er altijd voor me zijn. Majo, bedankt voor alle leuke momenten en goede gesprekken. Er gaat niets boven een avondje feesten met jou! (liefst gevolgd door een dagje shoppen en / of grens hoppen). Ik hoop dat er nog vele optredens zullen volgen in de toekomst. Vinz en Francien, ook met jullie kan ik mijn liefde voor muziek delen. We hebben al veel artiesten gezien, van Blistered tot ZZtop. Ik hoop dat er nog flink wat namen bij zullen komen op de lijst!

Papa en mama, jullie hebben me altijd de vrijheid gegeven om mijn eigen pad te kiezen. Bedankt voor jullie grenzeloze steun en vertrouwen. Dankzij jullie sta ik hier. Ik zeg het niet vaak, maar jullie betekenen alles voor me. Ook al woon ik inmiddels al jaren niet meer thuis, jullie zijn en blijven mijn veilige thuishaven. Oma, bedankt voor alle warmte en interesse. We wonen dan niet echt om de hoek en lopen de deur niet bij je plat, het is altijd gezellig als we langskomen en ik hoop dit nog flink wat jaartjes te kunnen doen.

Lieve Vince, m'n man, m'n beste vriend, m'n soulmate. Er zijn niet genoeg woorden om te omschrijven wat jij allemaal voor me hebt gedaan de afgelopen jaren. Bedankt voor je steun, hulp, vertrouwen, geduld, relativeringsvermogen, humor, inzichten, adviezen, en boven alles voor je onvoorwaardelijke liefde. Met jou aan mijn zij, kan ik de hele wereld aan. "Come what may. I will love you, until my dying day." 